\title{
The Real Jacobi Group Revisited
}

\author{
Stefan BERCEANU
}

National Institute for Physics and Nuclear Engineering, Department of Theoretical Physics, PO BOX MG-6, Bucharest-Magurele, Romania

E-mail: Berceanu@theory.nipne.ro

URL: http://www. theory.nipne.ro/index.php/mcp-home

Received May 09, 2019, in final form November 25, 2019; Published online December 07, 2019

https://doi.org/10.3842/SIGMA.2019.096

\begin{abstract}
The real Jacobi group $G_{1}^{J}(\mathbb{R})$, defined as the semi-direct product of the group $\mathrm{SL}(2, \mathbb{R})$ with the Heisenberg group $H_{1}$, is embedded in a $4 \times 4$ matrix realisation of the group $\operatorname{Sp}(2, \mathbb{R})$. The left-invariant one-forms on $G_{1}^{J}(\mathbb{R})$ and their dual orthogonal left-invariant vector fields are calculated in the S-coordinates $(x, y, \theta, p, q, \kappa)$, and a left-invariant metric depending of 4 parameters $(\alpha, \beta, \gamma, \delta)$ is obtained. An invariant metric depending of $(\alpha, \beta)$ in the variables $(x, y, \theta)$ on the Sasaki manifold $\mathrm{SL}(2, \mathbb{R})$ is presented. The well known Kähler balanced metric in the variables $(x, y, p, q)$ of the four-dimensional Siegel-Jacobi upper halfplane $\mathcal{X}_{1}^{J}=\frac{G_{1}^{J}(\mathbb{R})}{\operatorname{SO}(2) \times \mathbb{R}} \approx \mathcal{X}_{1} \times \mathbb{R}^{2}$ depending of $(\alpha, \gamma)$ is written down as sum of the squares of four invariant one-forms, where $\mathcal{X}_{1}$ denotes the Siegel upper half-plane. The left-invariant metric in the variables $(x, y, p, q, \kappa)$ depending on $(\alpha, \gamma, \delta)$ of a five-dimensional manifold $\tilde{\mathcal{X}}_{1}^{J}=\frac{G_{1}^{J}(\mathbb{R})}{\mathrm{SO}(2)} \approx \mathcal{X}_{1} \times \mathbb{R}^{3}$ is determined.
\end{abstract}

Key words: Jacobi group; invariant metric; Siegel-Jacobi upper half-plane; balanced metric; extended Siegel-Jacobi upper half-plane; naturally reductive manifold

2010 Mathematics Subject Classification: 32F45; 32Q15; 53C25; 53C22

\section{Introduction}

The Jacobi group [39, 55] of degree $n$ is defined as the semi-direct product $G_{n}^{J}=\mathrm{H}_{n} \rtimes \mathrm{Sp}(n, \mathbb{R})_{\mathbb{C}}$, where $\operatorname{Sp}(n, \mathbb{R})_{\mathbb{C}}=\operatorname{Sp}(n, \mathbb{C}) \cap \mathrm{U}(n, n)$ and $\mathrm{H}_{n}$ denotes the $(2 n+1)$-dimensional Heisenberg group $[16,17,108]$. To the Jacobi group $G_{n}^{J}$ it is associated a homogeneous manifold, called the SiegelJacobi ball $\mathcal{D}_{n}^{J}$ [16], whose points are in $\mathbb{C}^{n} \times \mathcal{D}_{n}$, i.e., a partially-bounded space. $\mathcal{D}_{n}$ denotes the Siegel (open) ball of degree $n$. The non-compact Hermitian symmetric space $\operatorname{Sp}(n, \mathbb{R})_{\mathbb{C}} / \mathrm{U}(n)$ admits a matrix realization as a homogeneous bounded domain [63]:

$$
\mathcal{D}_{n}:=\left\{W \in M(n, \mathbb{C}): W=W^{t}, \mathbb{1}_{n}-W \bar{W}>0\right\} .
$$

The Jacobi group is an interesting object in several branches of Mathematics, with important applications in Physics, see references in [13, 16, 21, 28, 29].

Our special interest to the Jacobi group comes from the fact that $G_{n}^{J}$ is a coherent state (CS) group [79, 80, 84, 85, 86, 87], i.e., a group which has orbits holomorphically embedded into a projective Hilbert space, for a precise definition see [12, Definition 1], [13], [22, Section 5.2.2] and [29, Remark 4.4]. To an element $X$ in the Lie algebra $\mathfrak{g}$ of $G$ we associated a first order differential operator $\mathbb{X}$ on the homogenous space $G / H$, with polynomial holomorphic coefficients, see $[23,24,25]$ for CS based on hermitian symmetric spaces, where the maximum degree of the polynomial is 2 . In $[12,26,27]$ we have advanced the hypothesis that for CS groups the coefficients in $\mathbb{X}$ are polynomial, and in [13] we have verified this for $G_{1}^{J}$. 
It was proved in $[16,17,21]$ that the Kähler two-form on $\mathcal{D}_{n}^{J}$, invariant to the action of the Jacobi group $G_{n}^{J}$, has the expression

$$
\begin{aligned}
& -\mathrm{i} \omega_{\mathcal{D}_{n}^{J}}(z, W)=\frac{k}{2} \operatorname{Tr}(\mathcal{B} \wedge \overline{\mathcal{B}})+\mu \operatorname{Tr}\left(\mathcal{A}^{t} \bar{M} \wedge \overline{\mathcal{A}}\right), \quad \mathcal{A}=\mathrm{d} z+\mathrm{d} W \bar{\eta}, \\
& \mathcal{B}=M \mathrm{~d} W, \quad M=\left(\mathbb{1}_{n}-W \bar{W}\right)^{-1}, \quad z \in \mathbb{C}^{n}, \quad W \in \mathcal{D}_{n}, \\
& \eta=M(z+W \bar{z}) .
\end{aligned}
$$

It was emphasized [17] that the change of coordinates $(z, W) \rightarrow(\eta, W)$, called FC-transform, has the meaning of passing from un-normalized to normalized Perelomov CS vectors [92]. Also, the FC-transform (1.2) is a homogeneous Kähler diffeomorphism from $\mathcal{D}_{n}^{J}$ to $\mathbb{C}^{n} \times \mathcal{D}_{n}$, in the meaning of the fundamental conjecture for homogeneous Kähler manifolds [53, 61, 103].

We reproduce a proposition which summarizes some of the geometric properties of the Jacobi group and the Siegel-Jacobi ball [21, 22], see the definitions of the notions appearing in the enunciation below in $[13,19,20,22]$ and also Appendix B for some notions on Berezin's quantization:

\section{Proposition 1.1.}

(i) The Jacobi group $G_{n}^{J}$ is a unimodular, non-reductive, algebraic group of Harish-Chandra type.

(ii) The Siegel-Jacobi domain $\mathcal{D}_{n}^{J}$ is a homogeneous, reductive, non-symmetric manifold associated to the Jacobi group $G_{n}^{J}$ by the generalized Harish-Chandra embedding.

(iii) The homogeneous Kähler manifold $\mathcal{D}_{n}^{J}$ is contractible.

(iv) The Kähler potential of the Siegel-Jacobi ball is global. $\mathcal{D}_{n}^{J}$ is a Q.-K. Lu manifold, with nowhere vanishing diastasis.

(v) The manifold $\mathcal{D}_{n}^{J}$ is a quantizable manifold.

(vi) The manifold $\mathcal{D}_{n}^{J}$ is projectively induced, and the Jacobi group $G_{n}^{J}$ is a CS-type group.

(vii) The Siegel-Jacobi ball $\mathcal{D}_{n}^{J}$ is not an Einstein manifold with respect to the balanced metric corresponding to the Kähler two-form (1.1), but it is one with respect to the Bergman metric corresponding to the Bergman Kähler two-form.

(viii) The scalar curvature is constant and negative.

The properties of geodesics on the Siegel-Jacobi disk $\mathcal{D}_{1}^{J}$ have been investigated in [13, 19, 20], while in [22] we have considered geodesics on the Siegel-Jacobi ball $\mathcal{D}_{n}^{J}$. We have explicitly determined the equations of geodesics on $\mathcal{D}_{n}^{J}$. We have proved that the FC-transform (1.2) is not a geodesic mapping on the non-symmetric space $\mathcal{D}_{n}^{J}$, see definition in [82].

However, it was not yet anlayzed whether the Siegel-Jacobi ball is a naturally reductive space or not, even if its points are in $\mathbb{C}^{n} \times \mathcal{D}_{n}$, both manifolds being naturally reductive, see Definition A.8 and Proposition A.9. In fact, this problem was the initial point of the present investigation. The answer to this question has significance in our approach [19] to the geometry of the Siegel-Jacobi ball via CS in the meaning of Perelomov [92]. We have proved in [8] that for symmetric manifolds the FC-transform gives geodesics, but the Siegel-Jacobi ball is not a symmetric space. Similar properties are expected for naturally reductive spaces $[9,10]$.

In the standard procedure of CS, see [16, 92], the Kähler two-form on a homogenous manifold $M$ is obtained from the Kähler potential $f(z, \bar{z})=\log K(z, \bar{z})$ via the recipe

$$
-\mathrm{i} \omega=\partial \bar{\partial} f
$$

where $K(z, z):=\left(e_{\bar{z}}, e_{\bar{z}}\right)$ is the scalar product of two CS at $z \in M$. In [21] we have underlined that the metric associated to the Kähler two-form (1.3) is a balanced metric, see more details in Appendix B. 
The real Jacobi group of degree $n$ is defined as $G_{n}^{J}(\mathbb{R}):=\operatorname{Sp}(n, \mathbb{R}) \ltimes H_{n}$, where $H_{n}$ is the real $(2 n+1)$-dimensional Heisenberg group. $\operatorname{Sp}(n, \mathbb{R})_{\mathbb{C}}$ and $G_{n}^{J}$ are isomorphic to $\operatorname{Sp}(n, \mathbb{R})$ and $G_{n}^{J}(\mathbb{R})$ respectively as real Lie groups, see [17, Proposition 2].

We have applied the partial Cayley transform from the Siegel-Jacobi ball to the Siegel-Jacobi upper half-plane and we have obtained the balanced metric on $\mathcal{X}_{n}^{J}$, see [17, Proposition 3].

However, the mentioned procedure of obtaining the invariant metric on homogeneous Kähler manifolds works only for even dimensional CS manifolds. For example, starting from the six dimensional real Jacobi group $G_{1}^{J}(\mathbb{R})=\mathrm{SL}(2, \mathbb{R}) \ltimes H_{1}$, we have obtained the Kähler invariant two-form $\omega_{\mathcal{X}_{1}^{J}}(2.4)$ on the Siegel-Jacobi upper half-plane, a four dimensional homogeneous manifold attached to the Jacobi group, $\mathcal{X}_{1}^{J}=\frac{G_{1}^{J}(\mathbb{R})}{\operatorname{SO}(2) \times \mathbb{R}} \approx \mathcal{X}_{1} \times \mathbb{R}^{2}[13,14,18,19]$, obtained previously by Berndt [37, 38], and Kähler [68, 69].

In the present paper we determine the invariant metric on a five dimensional homogeneous manifold, here called the extended Siegel-Jacobi upper half-plane, denoted $\tilde{\mathcal{X}}_{1}^{J}=\frac{G_{1}^{J}(\mathbb{R})}{\mathrm{SO}(2)} \approx$ $\mathcal{X}_{1} \times \mathbb{R}^{3}$. It will be important to find applications in Physics of the invariant metric (5.25) on the five-dimensional manifold $\tilde{\mathcal{X}}_{1}^{J}$.

In order to obtain invariant metric on odd dimensional manifolds, we are obliged to change our strategy applied previously to get the invariant metric on homogeneous Kähler manifolds. Instead of the mentioned first order differential operators on $M=G / H$ with holomorphic polynomial coefficients $\mathbb{X}$ associated to $X$ in the Lie algebra $\mathfrak{g}$ of $G[12,26]$, we have to use the fundamental vector field $X^{*}$ associated with $X$, see Appendix A. We have to abandon the approach in which the Jacobi algebra is defined as the semi-direct sum $\mathfrak{g}_{1}^{J}:=\mathfrak{h}_{1} \rtimes \mathfrak{s u}(1,1)$, where only the generators of $\mathfrak{s u}(1,1)$ have a matrix realization, see [13] and the summary in Section 2.

The approach of mathematicians is to consider the real Jacobi group $G_{1}^{J}(\mathbb{R})$ as subgroup of $\operatorname{Sp}(2, \mathbb{R})$. In the present paper we follow the notation in $[39,55]$ for the real Jacobi group $G_{1}^{J}(\mathbb{R})$, realized as submatrices of $\operatorname{Sp}(2, \mathbb{R})$ of the form

$$
\left(\begin{array}{cccc}
a & 0 & b & q \\
\lambda & 1 & \mu & \kappa \\
c & 0 & d & -p \\
0 & 0 & 0 & 1
\end{array}\right), \quad M=\left(\begin{array}{cc}
a & b \\
c & d
\end{array}\right), \quad \operatorname{det} M=1
$$

where

$$
Y:=(p, q)=X M^{-1}=(\lambda, \mu)\left(\begin{array}{ll}
a & b \\
c & d
\end{array}\right)^{-1}=(\lambda d-\mu c,-\lambda b+\mu a)
$$

is related to the Heisenberg group $H_{1}$.

To get the invariant metric on $\tilde{\mathcal{X}}_{1}^{J}$, we have determined the invariant one-forms $\lambda_{1}, \ldots, \lambda_{6}$ on $G_{1}^{J}(\mathbb{R})$, the main tool of the present paper, see details on the method in Appendix D.1.1. Then we have determined the invariant vector fields $L^{j}$ verifying the relations $\left\langle\lambda_{i} \mid L^{j}\right\rangle=\delta_{i j}$, $i, j=1, \ldots, 6$, such that $L^{j}$ are orthonormal with respect to the metric $\mathrm{d} s_{G_{1}^{J}(\mathbb{R})}^{2}$ in the $S$-variables $(x, y, \theta, p, q, \kappa)$, see [39, p. 10]. This is the idea of the method of the moving frame of E. Cartan $[49,50,56]$ explained in Section 5.4 .

Firstly, we recover the well known two-parameter balanced metric on $\mathcal{X}_{1}^{J}$ as sum of squares of the invariant one-forms $\lambda_{1}, \lambda_{2}, \lambda_{4}, \lambda_{5}$. Then the invariant metric on $\tilde{\mathcal{X}}_{1}^{J}$ is obtained as the sum of the squares of $\lambda_{1}, \lambda_{2}, \lambda_{4}, \ldots, \lambda_{6}$.

The paper is laid out as follows. In Section 2 we recall how we have obtained the Kähler two-forms on the Siegel-Jacobi disk $\mathcal{D}_{1}^{J}$ and on the Siegel-Jacobi upper half-plane $\mathcal{X}_{1}^{J}$, specifying the FC-transforms. Section 3 describes the real Heisenberg group $H_{1}$ embedded into 
$\operatorname{Sp}(2, \mathbb{R})$ : invariant one-forms, invariant metrics in the variables $(\lambda, \mu, \kappa)$. Note that in the formula (3.4) the last parenthesis $(\mathrm{d} \kappa-\mu \mathrm{d} \lambda+\lambda \mathrm{d} \mu)^{2}$ replaces $(\mathrm{d} \kappa)^{2}$ on the Euclidean space $\mathbb{R}^{3}(\kappa, \lambda, \mu)$ and the idea of the paper is to see the effect of this substitution in the invariant metric of the five-dimensional manifold $\tilde{\mathcal{X}}_{1}^{J}$. Section 4 deals with the $\operatorname{SL}(2, \mathbb{R})$ group as subgroup of $\operatorname{Sp}(2, \mathbb{R})$ in the variables $(x, y, \theta)$, which describe the Iwasawa decomposition. $\operatorname{SL}(2, \mathbb{R})$ is treated as a Sasaki manifold, with the invariant metric written down as sum of squares of the invariant one-forms $\lambda_{1}, \ldots, \lambda_{3}$ à la Milnor [83], while the metric on $\mathcal{X}_{1}$ is just $\lambda_{1}^{2}+\lambda_{2}^{2}$. Invariant metrics on $\mathrm{SL}(2, \mathbb{R})$ in other coordinates previously obtained by other authors are mentioned in Comment 4.2. Details on the calculations referring to $\mathrm{SL}(2, \mathbb{R})$ are presented also in Appendix C.3. Section 5 presents the real Jacobi group $G_{1}^{J}(\mathbb{R})$ in the EZ and S-coordinates [39]. The action of the reduced Jacobi group $G^{J}(\mathbb{R})_{0}$ on the four-dimensional manifold $\mathcal{X}_{1}^{J}$ is recalled $[13,14]$ and the fundamental vector fields on it are obtained. Also the action of $G_{1}^{J}(\mathbb{R})$ on the 5-dimensional manifold $\tilde{\mathcal{X}}_{1}^{J}$, called extended Siegel-Jacobi upper half-plane, is established in Lemma 5.1. The well known Kählerian balanced metric on the Siegel-Jacobi upper-half plane is written down as sum of the square of four invariant one-forms in Section 5.4. For this we have obtained the invariant one-forms on $G_{1}^{J}(\mathbb{R})$ in $(5.16)$. In Comment 5.5 we discuss the connection of our previous papers $[13,15,19]$ on $G_{1}^{J}(\mathbb{R})$ with the papers of Berndt $[37,38,39]$ and Kähler [68, 69], developed by Yang $[108,109,110,111,112]$ for $G_{n}^{J}(\mathbb{R})$. We have also determined the Killing vector fields as fundamental vector fields on the Siegel-Jacobi upper half-plane with the balanced metric $(5.21 \mathrm{~b})$. The same procedure is used to establish the invariant metric on the extended Siegel-Jacobi upper half-plane, which is not a Sasaki manifold. All the results concerning the invariant metrics on homogenous manifolds of dimensions 2-6 attached to the real Jacobi group of degree 1 are summarized in Theorem 5.7. As a consequence, we show by direct calculation that the Siegel-Jacobi upper half-plane is not a naturally reductive space with respect to the balanced metric, but it is one in the coordinates furnished by the FC-transform. In fact, this is the answer to the starting point of our investigation referring to the natural reductivity of $\mathcal{X}_{1}^{J}$. We also calculate the g.o. vectors [77] on $\mathcal{X}_{1}^{J}$ applying the geodesic Lemma A.19.

In four appendices we recall several basic mathematical concepts used in paper. Appendix A is devoted to naturally reductive spaces [51, 71, 88]. We have included the notions of Killing vectors, Riemannian homogeneous spaces [4], the list of 3 and 4-dimensional naturally reductive spaces $[35,36,76,100]$, the famous BCV-spaces $[41,48,104]$. Appendix B recalls the notion of balanced metric in the context of Berezin quantization. The Killing vectors on $S^{2}, \mathcal{D}_{1}, \mathbb{R}^{2}$ are presented in Appendix C. Appendix D refers to notions on Sasaki manifolds [42, 45, 95].

The main results of this paper are stated in Lemma 5.1, Remark 4.3, Propositions 4.1-5.8, and Theorem 5.7.

Notation. We denote by $\mathbb{R}, \mathbb{C}, \mathbb{Z}$, and $\mathbb{N}$ the field of real numbers, the field of complex numbers, the ring of integers, and the set of non-negative integers, respectively. We denote the imaginary unit $\sqrt{-1}$ by $\mathrm{i}$, and the Real and Imaginary part of a complex number by Re and respectively $\operatorname{Im}$, i.e., we have for $z \in \mathbb{C}, z=\operatorname{Re} z+\mathrm{i} \operatorname{Im} z$, and $\bar{z}=c c(z)=\operatorname{Re} z-\mathrm{i} \operatorname{Im} z$. We denote by $|M|$ or by $\operatorname{det}(M)$ the determinant of the matrix $M . M(n, m, \mathbb{F})$ denotes the set of $n \times m$ matrices with entries in the field $\mathbb{F}$. We denote by $M(n, \mathbb{F})$ the set $M(n, n, \mathbb{F})$. If $A \in M_{n}(\mathbb{F})$, then $A^{t}\left(A^{\dagger}\right)$ denotes the transpose (respectively, the Hermitian conjugate) of $A$. $\mathbb{1}_{n}$ denotes the identity matrix of degree $n$. We consider a complex separable Hilbert space $\mathfrak{H}$ endowed with a scalar product which is antilinear in the first argument, $(\lambda x, y)=\bar{\lambda}(x, y)$, $x, y \in \mathfrak{H}, \lambda \in \mathbb{C} \backslash 0$. We denote by "d" the differential. We use Einstein convention that repeated indices are implicitly summed over. The set of vector fields (1-forms) are denoted by $\mathfrak{D}^{1}\left(\mathfrak{D}_{1}\right)$. If $\lambda \in \mathfrak{D}_{1}$ and $L \in \mathfrak{D}^{1}$, then $\langle\lambda \mid L\rangle$ denotes their pairing. We use the symbol "Tr" to denote the trace of a matrix. If $X_{i}, i=1, \ldots, n$ are vectors in vector space $V$ over the field $\mathbb{F}$, then $\left\langle X_{1}, X_{2}, \ldots, X_{n}\right\rangle_{\mathbb{F}}$ denotes their span over $\mathbb{F}$. 


\section{The starting point in the coherent states approach}

We recall firstly our initial approach $[11,13]$ to the Jacobi group $G_{1}^{J}$ which we have followed in all our papers devoted to the Jacobi group, except [28] and [29]. The Lie algebra attached to $G_{1}^{J}$ is

$$
\mathfrak{g}_{1}^{J}:=\mathfrak{h}_{1} \rtimes \mathfrak{s u}(1,1),
$$

where $\mathfrak{h}_{1}$ is an ideal in $\mathfrak{g}_{1}^{J}$, i.e., $\left[\mathfrak{h}_{1}, \mathfrak{g}_{1}^{J}\right]=\mathfrak{h}_{1}$, determined by the commutation relations

$$
\begin{aligned}
& {\left[a, a^{\dagger}\right]=1,} \\
& {\left[K_{0}, K_{ \pm}\right]= \pm K_{ \pm}, \quad\left[K_{-}, K_{+}\right]=2 K_{0},} \\
& {\left[a, K_{+}\right]=a^{\dagger}, \quad\left[K_{-}, a^{\dagger}\right]=a,} \\
& {\left[K_{+}, a^{\dagger}\right]=\left[K_{-}, a\right]=0,} \\
& {\left[K_{0}, a^{\dagger}\right]=\frac{1}{2} a^{\dagger}, \quad\left[K_{0}, a\right]=-\frac{1}{2} a .}
\end{aligned}
$$

The Heisenberg algebra is

$$
\mathfrak{h}_{1} \equiv \mathfrak{g}_{H_{1}}=\left\langle\mathrm{i} s 1+x a^{\dagger}-\bar{x} a\right\rangle_{s \in \mathbb{R}, x \in \mathbb{C}}
$$

where $a^{\dagger}(a)$ are the boson creation (respectively, annihilation) operators which verify the canonical commutation relations (2.1a). The Lie algebra of the group $\mathrm{SU}(1,1)$ is

$$
\mathfrak{s u}(1,1)=\left\langle 2 \mathrm{i} \theta K_{0}+y K_{+}-\bar{y} K_{-}\right\rangle_{\theta \in \mathbb{R}, y \in \mathbb{C}},
$$

where the generators $K_{0}, K_{+}, K_{-}$verify the standard commutation relations (2.1b), and we have considered the matrix realization

$$
K_{0}=\frac{1}{2}\left(\begin{array}{cc}
1 & 0 \\
0 & -1
\end{array}\right), \quad K_{+}=\mathrm{i}\left(\begin{array}{cc}
0 & 1 \\
0 & 0
\end{array}\right), \quad K_{-}=\mathrm{i}\left(\begin{array}{cc}
0 & 0 \\
1 & 0
\end{array}\right) .
$$

We have determined the invariant metric on the Siegel-Jacobi upper half-plane $\mathcal{X}_{1}^{J}$ from the metric on $\mathcal{D}_{1}^{J}$ and the FC-transforms, see [13, 14, 18, 21]. For the actions in Proposition 2.1, where $G_{0}^{J}=\mathrm{SU}(1,1) \ltimes \mathbb{C}$, see $[17$, Proposition 2] and Lemma 5.1 below.

Proposition 2.1. Let us consider the Kähler two-form

$$
-\mathrm{i} \omega_{\mathcal{D}_{1}^{J}}(w, z)=\frac{2 k}{\left(1-|w|^{2}\right)^{2}} \mathrm{~d} w \wedge \mathrm{d} \bar{w}+\mu \frac{A \wedge \bar{A}}{1-|w|^{2}}, \quad A=A(w, z)=\mathrm{d} z+\bar{\eta} \mathrm{d} w,
$$

$G_{0}^{J}$-invariant to the action on the Siegel-Jacobi disk $\mathcal{D}_{1}^{J}$

$$
\left(\mathrm{SU}(1,1) \times \mathbb{C}^{2} \ni\left(\begin{array}{cc}
p & q \\
\bar{q} & \bar{p}
\end{array}\right), \alpha\right) \cdot(w, z)=\left(\frac{p w+q}{\bar{q} w+\bar{p}}, \frac{z+\alpha-\bar{\alpha} \omega}{\bar{q} w+\bar{p}}\right) .
$$

We have the homogeneous Kähler diffeomorphism $\mathrm{FC}:\left(\mathcal{D}_{1}^{J}, \omega_{\mathcal{D}_{1}^{J}}\right) \rightarrow\left(\mathcal{D}_{1}, \omega_{\mathcal{D}_{1}}\right) \oplus\left(\mathbb{C}, \omega_{\mathbb{C}}\right)$, $-\mathrm{i} \omega_{\mathbb{C}}=\mathrm{d} \eta \wedge \mathrm{d} \bar{\eta}$,

$$
\mathrm{FC}: \quad z=\eta-w \bar{\eta}, \quad \mathrm{FC}^{-1}: \eta=\frac{z+\bar{z} w}{1-|w|^{2}},
$$

and

$\mathrm{FC}: \quad A(w, z) \rightarrow \mathrm{d} \eta-w \mathrm{~d} \bar{\eta}$. 
The Kähler two-form (2.3) is invariant to the action $(g, \alpha) \times(\eta, w)=\left(\eta_{1}, w_{1}\right)$ of $G_{0}^{J}$ on $\mathbb{C} \times \mathcal{D}_{1}$ : $\eta_{1}=p(\eta+\alpha)+q(\bar{\eta}+\bar{\alpha})$.

Using the partial Cayley transform

$$
\begin{aligned}
& \Phi^{-1}: v=\mathrm{i} \frac{1+w}{1-w}, \quad u=\frac{z}{1-w}, \quad w, z \in \mathbb{C}, \quad|w|<1, \\
& \Phi: \quad w=\frac{v-\mathrm{i}}{v+\mathrm{i}}, \quad z=2 \mathrm{i} \frac{u}{v+\mathrm{i}}, \quad v, u \in \mathbb{C}, \quad \operatorname{Im} v>0,
\end{aligned}
$$

we get the Kähler two-form

$$
\begin{aligned}
& -\mathrm{i} \omega_{\mathcal{X}_{1}^{J}}(v, u)=\frac{2 k}{(\bar{v}-v)^{2}}+\frac{2 \mu}{\mathrm{i}(\bar{v}-v)} B \wedge \bar{B}, \\
& B(v, u)=A\left(\frac{v-\mathrm{i}}{v+\mathrm{i}}, \frac{2 \mathrm{i} u}{v+\mathrm{i}}\right)=\mathrm{d} u-\frac{u-\bar{u}}{v-\bar{v}} \mathrm{~d} v,
\end{aligned}
$$

$G^{J}(\mathbb{R})_{0}$-invariant to the action on the Siegel-Jacobi upper half-plane $\mathcal{X}_{1}^{J}$

$$
\left(\mathrm{SL}(2, \mathbb{R}) \times \mathbb{C}^{2} \ni\left(\begin{array}{ll}
a & b \\
c & d
\end{array}\right), \alpha\right) \cdot(v, u)=\left(\frac{a v+b}{c v+d}, \frac{u+n v+m}{c v+d}\right), \quad \alpha=m+\mathrm{i} n .
$$

We have the homogeneous Kähler diffeomorphism

$$
\begin{aligned}
& \mathrm{FC}_{1}: \quad\left(\mathcal{X}_{1}^{J}, \omega_{\mathcal{X}_{1}^{J}}\right) \rightarrow\left(\mathcal{X}_{1}, \omega_{\mathcal{D}_{1}}\right) \oplus\left(\mathbb{C}, \omega_{\mathbb{C}}\right), \\
& \mathrm{FC}_{1}: 2 \mathrm{i} u=(v+\mathrm{i}) \eta-(v-\mathrm{i}) \bar{\eta}, \quad \mathrm{FC}_{1}^{-1}: \quad \eta=\frac{u \bar{v}-\bar{u} v+\mathrm{i}(\bar{u}-u)}{\bar{v}-v} .
\end{aligned}
$$

The situation is summarized in the commutative diagram of the table FC-transforms

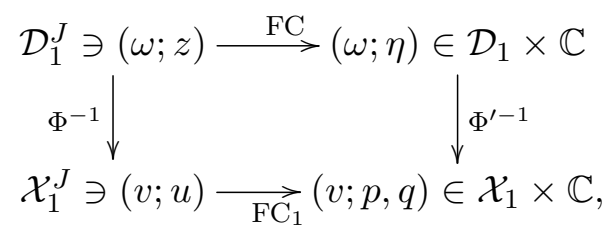

where

$$
\Phi^{\prime-1}: \eta \rightarrow q+\mathrm{i} p, \quad \Phi^{\prime}:(q, p) \rightarrow \eta=q+\mathrm{i} p .
$$

We recall that in Proposition 2.1 the parameters $k$ and $\mu$ come from representation theory of the Jacobi group: $k$ indexes the positive discrete series of $\operatorname{SU}(1,1)(2 k \in \mathbb{N})$, while $\mu>0$ indexes the representations of the Heisenberg group. Note that in the Berndt-Kähler approach the Kähler potential (5.24) is just "guessed", see Comment 5.5.

Here we just verify the invariance of the Kähler two-form (2.4) to the action (2.5), see also Lemma 5.1. We use equations (2.6)

$$
\mathrm{d} v_{1}=\frac{\mathrm{d} v}{\Lambda^{2}}, \quad v_{1}-\bar{v}_{1}=\frac{v-\bar{v}}{|\Lambda|^{2}}, \quad \text { where } \quad \Lambda=c v+d,
$$

and the particular case $n=1$ of equations in $[17$, p. 17]

$$
\mathrm{d} u_{1}=\frac{\mathrm{d} u}{\Lambda}+\frac{n d-c(u+m)}{\Lambda^{2}} \mathrm{~d} v, \quad B_{1}=\frac{B}{\Lambda},
$$

where $B$ is given in (2.4). 


\section{The Heisenberg subgroup of $\operatorname{Sp}(2, \mathbb{R})$}

The composition law of the 3 -dimensional Heisenberg group $H_{1}$ in (1.4) is:

$$
(\lambda, \mu, \kappa)\left(\lambda^{\prime}, \mu^{\prime}, \kappa^{\prime}\right)=\left(\lambda+\lambda^{\prime}, \mu+\mu^{\prime}, \kappa+\kappa^{\prime}+\lambda \mu^{\prime}-\lambda^{\prime} \mu\right) .
$$

We denote an element of $H_{1}$ embedded in $\operatorname{Sp}(2, \mathbb{R})$ as in (1.4) with $M=\mathbb{1}_{2}$

$$
H_{1} \ni g=\left(\begin{array}{cccc}
1 & 0 & 0 & \mu \\
\lambda & 1 & \mu & \kappa \\
0 & 0 & 1 & -\lambda \\
0 & 0 & 0 & 1
\end{array}\right), \quad g^{-1}=\left(\begin{array}{cccc}
1 & 0 & 0 & -\mu \\
-\lambda & 1 & -\mu & -\kappa \\
0 & 0 & 1 & \lambda \\
0 & 0 & 0 & 1
\end{array}\right) .
$$

A base of the Lie algebra $\mathfrak{h}_{1}=\langle P, Q, R\rangle_{\mathbb{R}}$ of the Heisenberg group $H_{1}$ in the realization (3.1) in the space $M(4, \mathbb{R})$ consists of the matrices

$$
P=\left(\begin{array}{cccc}
0 & 0 & 0 & 0 \\
1 & 0 & 0 & 0 \\
0 & 0 & 0 & -1 \\
0 & 0 & 0 & 0
\end{array}\right), \quad Q=\left(\begin{array}{cccc}
0 & 0 & 0 & 1 \\
0 & 0 & 1 & 0 \\
0 & 0 & 0 & 0 \\
0 & 0 & 0 & 0
\end{array}\right), \quad R=\left(\begin{array}{cccc}
0 & 0 & 0 & 0 \\
0 & 0 & 0 & 1 \\
0 & 0 & 0 & 0 \\
0 & 0 & 0 & 0
\end{array}\right)
$$

which verify the commutation relations

$$
[P, Q]=2 R, \quad[P, R]=[Q, R]=0 .
$$

If we write

$$
H_{1} \ni g(\lambda, \mu, \kappa)=\mathbb{1}_{4}+\lambda P+\mu Q+\kappa R,
$$

then, using the formulas, see details in Appendix D.1,

$$
g^{-1} \mathrm{~d} g=P \lambda^{p}+Q \lambda^{q}+R \lambda^{r}, \quad \mathrm{~d} g g^{-1}=P \rho^{p}+Q \rho^{q}+R \rho^{r},
$$

we find the left-invariant one-forms (vector fields)

$$
\left\{\begin{array} { l } 
{ \lambda ^ { p } = \mathrm { d } \lambda , } \\
{ \lambda ^ { q } = \mathrm { d } \mu , } \\
{ \lambda ^ { r } = \mathrm { d } \kappa - \lambda \mathrm { d } \mu + \mu \mathrm { d } \lambda , }
\end{array} \quad \left\{\begin{array}{l}
L^{p}=\partial_{\lambda}-\mu \partial_{\kappa}, \\
L^{q}=\partial_{\mu}+\lambda \partial_{\kappa}, \\
L^{r}=\partial_{\kappa},
\end{array}\right.\right.
$$

and the right-invariant one-forms (respectively vector fields)

$$
\left\{\begin{array} { l } 
{ \rho ^ { p } = \mathrm { d } \lambda , } \\
{ \rho ^ { q } = \mathrm { d } \mu , } \\
{ \rho ^ { r } = \mathrm { d } \kappa - \mu \mathrm { d } \lambda + \lambda \mathrm { d } \mu , }
\end{array} \quad \left\{\begin{array}{l}
R^{p}=\partial_{\lambda}+\mu \partial_{\kappa}, \\
R^{q}=\partial_{\mu}-\lambda \partial_{\kappa}, \\
R^{r}=\partial_{\kappa} .
\end{array}\right.\right.
$$

We have the commutation relations

$$
\left[L^{p}, L^{q}\right]=2 L^{r}, \quad\left[R^{p}, R^{q}\right]=-2 R^{r} .
$$

We get the right (respectively left) invariant metric on $H_{1}$

$$
\begin{aligned}
& g_{H_{1}}^{R}(\lambda, \mu, \kappa)=\left(\rho^{p}\right)^{2}+\left(\rho^{q}\right)^{2}+\left(\rho^{r}\right)^{2}=\mathrm{d} \lambda^{2}+\mathrm{d} \mu^{2}+(\mathrm{d} \kappa-\mu \mathrm{d} \lambda+\lambda \mathrm{d} \mu)^{2}, \\
& g_{H_{1}}^{L}(\lambda, \mu, \kappa)=\left(\lambda^{p}\right)^{2}+\left(\lambda^{q}\right)^{2}+\left(\lambda^{r}\right)^{2}=\mathrm{d} \lambda^{2}+\mathrm{d} \mu^{2}+(\mathrm{d} \kappa-\lambda \mathrm{d} \mu+\mu \mathrm{d} \lambda)^{2} .
\end{aligned}
$$

The left (right) invariant action of $H_{1}$ on itself is given by

$$
\begin{aligned}
& \exp (\lambda P+\mu Q+\kappa R)\left(\lambda_{0}, \mu_{0}, \kappa_{0}\right)=\left(\lambda+\lambda_{0}, \mu+\mu_{0}, \kappa+\kappa_{0}+\lambda \mu_{0}-\mu \lambda_{0}\right), \\
& \left(\lambda_{0}, \mu_{0}, \kappa_{0}\right) \exp (\lambda P+\mu Q+\kappa R)=\left(\lambda+\lambda_{0}, \mu+\mu_{0}, \kappa+\kappa_{0}+\lambda_{0} \mu-\mu_{0} \lambda\right) .
\end{aligned}
$$

With (3.5), we calculate the fundamental vector fields

$$
P^{*}=\partial_{\lambda}+\mu \partial_{\kappa}, \quad Q^{*}=\partial_{\mu}-\lambda \partial_{\kappa}, \quad R^{*}=\partial_{\kappa} .
$$




\section{The $\operatorname{SL}(2, \mathbb{R})$ subgroup of $\operatorname{Sp}(2, \mathbb{R})$}

An element $M \in \mathrm{SL}(2, \mathbb{R})$ is realized as an element in $\operatorname{Sp}(2, \mathbb{R})$ by the relation

$$
M=\left(\begin{array}{ll}
a & b \\
c & d
\end{array}\right) \rightarrow g=\left(\begin{array}{cccc}
a & 0 & b & 0 \\
0 & 1 & 0 & 0 \\
c & 0 & d & 0 \\
0 & 0 & 0 & 1
\end{array}\right) \in G_{1}^{J}(\mathbb{R}), \quad g^{-1}=\left(\begin{array}{cccc}
d & 0 & -b & 0 \\
0 & 1 & 0 & 0 \\
-c & 0 & a & 0 \\
0 & 0 & 0 & 1
\end{array}\right)
$$

A basis of the Lie algebra $\mathfrak{s l}(2, \mathbb{R})=\langle F, G, H\rangle_{\mathbb{R}}$ consists of the matrices in $M(4, \mathbb{R})$

$$
F=\left(\begin{array}{cccc}
0 & 0 & 1 & 0 \\
0 & 0 & 0 & 0 \\
0 & 0 & 0 & 0 \\
0 & 0 & 0 & 0
\end{array}\right), \quad G=\left(\begin{array}{cccc}
0 & 0 & 0 & 0 \\
0 & 0 & 0 & 0 \\
1 & 0 & 0 & 0 \\
0 & 0 & 0 & 0
\end{array}\right), \quad H=\left(\begin{array}{cccc}
1 & 0 & 0 & 0 \\
0 & 0 & 0 & 0 \\
0 & 0 & -1 & 0 \\
0 & 0 & 0 & 0
\end{array}\right) .
$$

$F, G, H$ verify the commutation relations (C.11). With the representation (4.1), we have

$$
g^{-1} \mathrm{~d} g=F \lambda^{f}+G \lambda^{g}+H \lambda^{h}, \quad \mathrm{~d} g g^{-1}=F \rho^{f}+G \rho^{g}+H \rho^{h} .
$$

Using the parameterization (4.1) for $\operatorname{SL}(2, \mathbb{R})$, we find

$$
\left\{\begin{array} { l } 
{ \lambda ^ { f } = d \mathrm { d } b - b \mathrm { d } d , } \\
{ \lambda ^ { g } = - c \mathrm { d } a + a \mathrm { d } c , } \\
{ \lambda ^ { h } = d \mathrm { d } a - b \mathrm { d } c = c \mathrm { d } b - a \mathrm { d } d , }
\end{array} \quad \left\{\begin{array}{l}
\rho^{f}=-b \mathrm{~d} a+a \mathrm{~d} b \\
\rho^{g}=d \mathrm{~d} c-c \mathrm{~d} d \\
\rho^{h}=d \mathrm{~d} a-c \mathrm{~d} b
\end{array}\right.\right.
$$

We use the notation of [39, Section 1.4]. The Iwasawa decomposition $M=N A K$ of an element $M$ as in (4.1) reads

$$
M=\left(\begin{array}{ll}
1 & x \\
0 & 1
\end{array}\right)\left(\begin{array}{cc}
y^{\frac{1}{2}} & 0 \\
0 & y^{-\frac{1}{2}}
\end{array}\right)\left(\begin{array}{cc}
\cos \theta & \sin \theta \\
-\sin \theta & \cos \theta
\end{array}\right), \quad y>0 .
$$

Comparing (4.3) with (4.1), we find

$$
\begin{aligned}
& a=y^{1 / 2} \cos \theta-x y^{-1 / 2} \sin \theta, \quad b=y^{1 / 2} \sin \theta+x y^{-1 / 2} \cos \theta, \\
& c=-y^{-1 / 2} \sin \theta, \quad d=y^{-1 / 2} \cos \theta
\end{aligned}
$$

and

$$
x=\frac{a c+b d}{d^{2}+c^{2}}, \quad y=\frac{1}{d^{2}+c^{2}}, \quad \sin \theta=-\frac{c}{\sqrt{c^{2}+d^{2}}}, \quad \cos \theta=\frac{d}{\sqrt{c^{2}+d^{2}}} .
$$

From (4.4), we get the differentials

$$
\begin{aligned}
\mathrm{d} a & =-y^{-\frac{1}{2}} \sin \theta \mathrm{d} x+\frac{1}{2 y^{\frac{1}{2}}}\left(\cos \theta+\frac{x}{y} \sin \theta\right) \mathrm{d} y-y^{\frac{1}{2}}\left(\sin \theta+\frac{x}{y} \cos \theta\right) \mathrm{d} \theta, \\
\mathrm{d} b & =y^{-\frac{1}{2}} \cos \theta \mathrm{d} x+\frac{1}{2 y^{\frac{1}{2}}}\left(\sin \theta-\frac{x}{y} \cos \theta\right) \mathrm{d} y+y^{\frac{1}{2}}\left(\cos \theta-\frac{x}{y} \sin \theta\right) \mathrm{d} \theta \\
\mathrm{d} c & =y^{-1 / 2}\left(\frac{\sin \theta}{2 y} \mathrm{~d} y-\cos \theta \mathrm{d} \theta\right), \quad \mathrm{d} d=-y^{-1 / 2}\left(\frac{\cos \theta}{2 y} \mathrm{~d} y+\sin \theta \mathrm{d} \theta\right) .
\end{aligned}
$$

Let $M, M^{\prime}, M_{1} \in \mathrm{SL}(2, \mathbb{R})$ such that $M M^{\prime}=M_{1}$, matrices of the form (4.1). With (4.4) we calculate the explicit action of $M \in \mathrm{SL}(2, \mathbb{R})$ on $\left(x^{\prime}, y^{\prime}, \theta^{\prime}\right)$

$$
x_{1}+\mathrm{i} y_{1}=\frac{\left(a x^{\prime}+b\right)\left(c x^{\prime}+d\right)+a c y^{\prime 2}+\mathrm{i} y^{\prime}}{\Lambda}, \quad \Lambda=\left(c x^{\prime}+d\right)^{2}+\left(c y^{\prime}\right)^{2},
$$




$$
\sin \theta_{1}=\frac{\left(c x^{\prime}+d\right) \sin \theta^{\prime}-c y^{\prime} \cos \theta^{\prime}}{\sqrt{\Lambda}}, \quad \cos \theta_{1}=\frac{c y^{\prime} \sin \theta^{\prime}+\left(c x^{\prime}+d\right) \cos \theta^{\prime}}{\sqrt{\Lambda}} .
$$

With (4.7), we find out

$$
\begin{aligned}
\frac{\mathrm{d} x_{1}}{y_{1}} & =\frac{\left[\left(c x^{\prime}+d\right)^{2}-\left(c y^{\prime}\right)^{2}\right] \mathrm{d} x^{\prime}+2 c y^{\prime}\left(c x^{\prime}+d\right) \mathrm{d} y^{\prime}}{y^{\prime} \Lambda}, \\
\frac{\mathrm{d} y_{1}}{y_{1}} & =\frac{-2 c y^{\prime}\left(c x^{\prime}+d\right) \mathrm{d} x^{\prime}+\left[\left(c x^{\prime}+d\right)^{2}-\left(c y^{\prime}\right)^{2}\right] \mathrm{d} y^{\prime}}{y^{\prime} \Lambda}, \\
\mathrm{d} \theta_{1} & =\mathrm{d} \theta^{\prime}+c \frac{c y^{\prime} \mathrm{d} x^{\prime}-\left(c x^{\prime}+d\right) \mathrm{d} y^{\prime}}{\Lambda} .
\end{aligned}
$$

Introducing (4.4) and (4.6) into (4.2), we find the left (right)-invariant one-forms $\lambda$-s with respect to the action $M \cdot\left(x^{\prime}, y^{\prime}, \theta^{\prime}\right)=\left(x_{1}, y_{1}, \theta_{1}\right), M \in \mathrm{SL}(2, \mathbb{R})$ and $\left(x_{1}, y_{1}, \theta_{1}\right)$ given by (4.7) (respectively $\rho$-s),

$$
\left\{\begin{array} { l } 
{ \lambda ^ { f } = \frac { \mathrm { d } x } { y } \operatorname { c o s } ^ { 2 } \theta + \frac { \mathrm { d } y } { 2 y } \operatorname { s i n } 2 \theta + \mathrm { d } \theta , } \\
{ \lambda ^ { g } = - \frac { \mathrm { d } x } { y } \operatorname { s i n } ^ { 2 } \theta + \frac { \mathrm { d } y } { 2 y } \operatorname { s i n } 2 \theta - \mathrm { d } \theta , } \\
{ \lambda ^ { h } = - \frac { \mathrm { d } x } { 2 y } \operatorname { s i n } 2 \theta + \frac { \mathrm { d } y } { 2 y } \operatorname { c o s } 2 \theta , }
\end{array} \quad \left\{\begin{array}{l}
\rho^{f}=\mathrm{d} x-\frac{x}{y} \mathrm{~d} y+\frac{x^{2}+y^{2}}{y} \mathrm{~d} \theta, \\
\rho^{g}=-\frac{\mathrm{d} \theta}{y}, \\
\rho^{h}=\frac{\mathrm{d} y}{2 y}-\frac{x}{y} \mathrm{~d} \theta .
\end{array}\right.\right.
$$

We determine the left-invariant vector fields $L^{f}, L^{g}, L^{h}$ on $\operatorname{SL}(2, \mathbb{R})$, dual orthogonal to the left-invariant one-forms $\lambda^{f}, \lambda^{g}, \lambda^{h}(4.8)$

$$
\begin{aligned}
L^{f} & =y \cos 2 \theta \frac{\partial}{\partial x}+y \sin 2 \theta \frac{\partial}{\partial y}+\sin ^{2} \theta \frac{\partial}{\partial \theta}, \\
L^{g} & =y \cos 2 \theta \frac{\partial}{\partial x}+y \sin 2 \theta \frac{\partial}{\partial y}-\cos ^{2} \theta \frac{\partial}{\partial \theta}, \\
L^{h} & =-2 y \sin 2 \theta \frac{\partial}{\partial x}+2 y \cos 2 \theta \frac{\partial}{\partial y}+\sin 2 \theta \frac{\partial}{\partial \theta},
\end{aligned}
$$

which verify the commutation relations (C.11) of the generators $F, G, H$ of the Lie algebra $\mathfrak{s l}(2, \mathbb{R})$. In fact, $L^{f}, L^{g}, L^{h}$ in (4.9) give the Lie derivative of the matrices $F, G$, respectively $H$, see, e.g., [78, p. 114].

From (4.8), we also get

$$
\begin{aligned}
& \lambda^{f}+\lambda^{g}=\frac{1}{y}(\cos 2 \theta \mathrm{d} x+\sin 2 \theta \mathrm{d} y), \\
& 2 \lambda^{h}=\frac{1}{y}(-\sin 2 \theta \mathrm{d} x+\cos 2 \theta \mathrm{d} y), \\
& \lambda^{f}-\lambda^{g}=\frac{\mathrm{d} x}{y}+2 \mathrm{~d} \theta,
\end{aligned}
$$

and

$$
\begin{aligned}
& \frac{1}{2}\left(L^{f}+L^{g}\right)=y \cos 2 \theta \frac{\partial}{\partial x}+y \sin 2 \theta \frac{\partial}{\partial y}-\frac{1}{2} \cos 2 \theta \frac{\partial}{\partial \theta}, \\
& \frac{1}{2}\left(L^{f}-L^{g}\right)=\frac{1}{2} \frac{\partial}{\partial \theta}, \\
& \frac{1}{2} L^{h}=-y \sin 2 \theta \frac{\partial}{\partial x}+y \cos 2 \theta \frac{\partial}{\partial y}+\frac{1}{2} \sin 2 \theta \frac{\partial}{\partial \theta} .
\end{aligned}
$$


Taking $\alpha, \beta>0$, let us introduce the left invariant one-forms

$$
\begin{aligned}
& \lambda_{1}=\sqrt{\alpha}\left(\lambda^{f}+\lambda^{g}\right)=\frac{\sqrt{\alpha}}{y}(\cos 2 \theta \mathrm{d} x+\sin 2 \theta \mathrm{d} y), \\
& \lambda_{2}=2 \sqrt{\alpha} \lambda^{h}=\frac{\sqrt{\alpha}}{y}(-\sin 2 \theta \mathrm{d} x+\cos 2 \theta \mathrm{d} y), \\
& \lambda_{3}=\sqrt{\beta}\left(\lambda^{f}-\lambda^{g}\right)=\sqrt{\beta}\left(\frac{\mathrm{d} x}{y}+2 \mathrm{~d} \theta\right) .
\end{aligned}
$$

The parameters $\alpha, \beta$ introduced in (4.10) will appear in the invariant metrics $(4.21)$ on $\mathrm{SL}(2, \mathbb{R})$ and $(5.27)$ on $G_{1}^{J}(\mathbb{R})$, while only $\alpha$ will appear in the invariant metrics (5.22) on $\mathcal{X}_{1}^{J}$ and (5.25) on $\tilde{\mathcal{X}}_{1}^{J}$.

Note that in the commutation relations of the generators $e_{1}, e_{2}, e_{3}$ of the Lie algebra $\mathfrak{s l}(2, \mathbb{R})$

$$
\left[e_{1}, e_{2}\right]=-4 \frac{\alpha}{\sqrt{\beta}} e_{3}, \quad\left[e_{2}, e_{3}\right]=4 \sqrt{\beta} e_{1}, \quad\left[e_{3}, e_{1}\right]=\sqrt{\beta} e_{2},
$$

where

$$
e_{1}=\sqrt{\alpha}(F+G), \quad e_{2}=2 \sqrt{\alpha} H, \quad e_{3}=\sqrt{\beta}(F-G),
$$

there are 2 positive structure constants and one negative, as in the scheme of classification of three-dimensional unimodular Lie groups, see [83, p. 307].

We determine the left-invariant vector fields $L^{j}$ such that $\left\langle\lambda_{i} \mid L^{j}\right\rangle=\delta_{i j}, i, j=1,2,3$,

$$
\begin{aligned}
L^{1} & =\frac{1}{2 \sqrt{\alpha}}\left(L^{f}+L^{g}\right)=\frac{1}{\sqrt{\alpha}}\left(y \cos 2 \theta \frac{\partial}{\partial x}+y \sin 2 \theta \frac{\partial}{\partial y}-\frac{1}{2} \cos 2 \theta \frac{\partial}{\partial \theta}\right), \\
L^{2} & =\frac{1}{2 \sqrt{\alpha}} L^{h}=\frac{1}{\sqrt{\alpha}}\left(-y \sin 2 \theta \frac{\partial}{\partial x}+y \cos 2 \theta \frac{\partial}{\partial y}+\frac{1}{2} \sin 2 \theta \frac{\partial}{\partial \theta}\right), \\
L^{3} & =\frac{1}{2 \sqrt{\beta}}\left(L^{f}-L^{g}\right)=\frac{1}{2 \sqrt{\beta}} \frac{\partial}{\partial \theta} .
\end{aligned}
$$

If we take in (4.11) the limit $\theta \rightarrow 0$, we project the invariant vector fields of $\operatorname{SL}(2, \mathbb{R})$ on the Siegel half-plane $\mathcal{X}_{1}=\left\{(x, y) \in \mathbb{R}^{2} \mid y>0\right\}$, and we recover the invariant vector fields which appear in Theorem A.10(2) equation (A.11)

$$
l_{0}^{1}=\frac{y}{\sqrt{\alpha}} \frac{\partial}{\partial x}, \quad l_{0}^{2}=\frac{y}{\sqrt{\alpha}} \frac{\partial}{\partial y} .
$$

Now we calculate the fundamental vector fields $f^{*}, g^{*}, h^{*}$ of manifold $\operatorname{SL}(2, \mathbb{R})$ attached to the base $F, G$, respectively $H$, invariant to the action $(x, y, \theta) \cdot\left(x^{\prime}, y^{\prime}, \theta^{\prime}\right)=\left(x_{1}, y_{1}, z_{1}\right)$ given by the composition law $M M^{\prime}=M_{1}$, applying (C.21), (4.4), (4.5):

$$
\begin{aligned}
f^{*} & =F_{1}^{*}=\frac{\partial}{\partial x}, \\
g^{*} & =G_{1}^{*}-y \frac{\partial}{\partial \theta}=\left(y^{2}-x^{2}\right) \frac{\partial}{\partial x}-2 x y \frac{\partial}{\partial y}-y \frac{\partial}{\partial \theta}, \\
h^{*} & =H_{1}^{*}=2\left(x \frac{\partial}{\partial x}+y \frac{\partial}{\partial y}\right),
\end{aligned}
$$

where we have denoted with a subindex 1 the fundamental vector fields (C.23) of $\mathrm{SL}(2, \mathbb{R})$, corresponding to the action of the group on the Siegel upper half-plane $\mathcal{X}_{1}$. In fact, $F_{1}^{*}, G_{1}^{*}, H_{1}^{*}$ are $\mathbb{F}, \mathbb{G}, \mathbb{H}$ in the convention of Section 1. Evidently, the vector fields (4.13) verify the same commutation relations as $F, G, H$, with a minus sign. 
Using (C.22) or directly with (4.13), we calculate the fundamental vector fields $v^{*}, h 1^{*}, w^{*}$ of $\operatorname{SL}(2, \mathbb{R})$ corresponding to

$$
\begin{aligned}
& v=\sqrt{\alpha}(F+G), \quad h 1=2 \sqrt{\alpha} H, \quad w=\sqrt{\beta}(F-G), \\
& v^{*}=\sqrt{\alpha}\left[\left(1-x^{2}+y^{2}\right) \frac{\partial}{\partial x}-2 x y \frac{\partial}{\partial y}-y \frac{\partial}{\partial \theta}\right], \\
& h 1^{*}=4 \sqrt{\alpha}\left(x \frac{\partial}{\partial x}+y \frac{\partial}{\partial y}\right), \\
& w^{*}=\sqrt{\beta}\left[\left(1+x^{2}-y^{2}\right) \frac{\partial}{\partial x}+2 x y \frac{\partial}{\partial y}+y \frac{\partial}{\partial \theta}\right] .
\end{aligned}
$$

Now we consider $\operatorname{SL}(2, \mathbb{R})$ as a contact manifold in the meaning of Definition D.3. Firstly we define an almost contact structure $(\Phi, \xi, \eta)$ as in Definition D.1. We take $\eta=\lambda_{3}$ and $\xi=L^{3}$, verifying (D.3a). We have $\mathrm{d} \eta=\mathrm{d} \lambda_{3}=\sqrt{\beta} \frac{\mathrm{d} x \wedge \mathrm{d} y}{y^{2}}$, and the condition (D.6) (with $n=1$ ) that $\eta$ be a contact form is verified. The only nonzero component of the associated two form $\hat{\Phi}$ in (D.7) is $\hat{\Phi}_{x y}=\frac{\sqrt{\beta}}{2 y^{2}}$, i.e.,

$$
\hat{\Phi}=\left(\begin{array}{ccc}
\hat{\Phi}_{x x} & \hat{\Phi}_{x y} & \hat{\Phi}_{x \theta} \\
\hat{\Phi}_{y x} & \hat{\Phi}_{y y} & \hat{\Phi}_{y \theta} \\
\hat{\Phi}_{\theta x} & \hat{\Phi}_{\theta y} & \hat{\Phi}_{\theta \theta}
\end{array}\right)=\left(\begin{array}{ccc}
0 & \frac{\sqrt{\beta}}{2 y^{2}} & 0 \\
-\frac{\sqrt{\beta}}{2 y^{2}} & 0 & 0 \\
0 & 0 & 0
\end{array}\right)
$$

From (D.5) applied to the metric matrix $(4.21)$ of $\mathrm{SL}(2, \mathbb{R})$, we get

$$
\Phi=\left(\begin{array}{ccc}
\Phi_{x}^{x} & \Phi_{y}^{x} & \Phi_{\theta}^{x} \\
\Phi_{x}^{y} & \Phi_{y}^{y} & \Phi_{\theta}^{y} \\
\Phi_{x}^{\theta} & \Phi_{y}^{\theta} & \Phi_{\theta}^{\theta}
\end{array}\right)=\left(\begin{array}{ccc}
0 & \frac{\sqrt{\beta}}{2 \alpha} & 0 \\
-\frac{\sqrt{\beta}}{2 \alpha} & 0 & 0 \\
0 & -\frac{\sqrt{\beta}}{4 \alpha y} & 0
\end{array}\right) .
$$

It is convenient to work with the matrix

$$
\Phi^{\prime}=\frac{2 \alpha}{\sqrt{\beta}} \Phi=\left(\begin{array}{ccc}
0 & 1 & 0 \\
-1 & 0 & 0 \\
0 & -\frac{1}{2 y} & 0
\end{array}\right) .
$$

With $(\eta, \xi, \Phi)$ chosen as $\left(\lambda_{3}, L^{3}, \Phi^{\prime}\right)$, equation (D.3b) and the conditions of Theorem D.2 for an almost contact structure for the manifold $\operatorname{SL}(2, \mathbb{R})$, where $\operatorname{Rank}(\Phi)=2$, are verified.

We have

$$
\operatorname{Ker}(\eta)=\left\langle V_{1}, V_{2}\right\rangle=\left\langle\frac{\partial}{\partial x}-\frac{1}{2 y} \frac{\partial}{\partial \theta}, \frac{\partial}{\partial y}\right\rangle,
$$

and we can write the $(1,1)$-tensor $\Phi^{\prime}(\mathrm{D} .2)$ as

$$
\Phi^{\prime}=-V_{2} \otimes \mathrm{d} x+V_{1} \otimes \mathrm{d} y .
$$

If $X=A \frac{\partial}{\partial x}+B \frac{\partial}{\partial y}+C \frac{\partial}{\partial \theta}$, then $\Phi^{\prime} X=B V_{1}-A V_{2}$, and the contact distribution $\mathcal{D}=\left\langle V_{1}, V_{2}\right\rangle$ verifies the condition of Remark D.4.

We also observe that $\mathrm{SL}(2, \mathbb{R})$ is a homogeneous contact manifold in the sense of Definition D.5. 
Now we construct the 4 -dimensional symplectization $(C(\mathrm{SL}(2, \mathbb{R})), \omega, \bar{g})$ of $\mathrm{SL}(2, \mathbb{R})$, where

$$
\bar{g}(r, x, y, \theta)=\mathrm{d} r^{2}+r^{2} g_{\mathrm{SL}(2, \mathbb{R})}(x, y, \theta), \quad \omega=\mathrm{d}\left(r^{2} \lambda_{3}\right) .
$$

In order to see that the Riemann cone $(C(\mathrm{SL}(2, \mathbb{R})), \omega, \bar{g})$ of the manifold $\mathrm{SL}(2, \mathbb{R})$ is normal in the sense of Definition D.10, we calculate the components (D.17) of the (1,2)-tensor $N^{1}$ (D.9), using equations (4.15) and (4.16). Because the tensor (D.10) is antisymmetric in the lower indexes $i, j$, we have to calculate only the 9 components $\left(N^{1}\right)_{x, y}^{i},\left(N^{1}\right)_{x, \theta}^{i},\left(N^{1}\right)_{y, \theta}^{i}, i=x, y, \theta$, which were found to be 0 . In accord with Definition D.10, the Riemann cone $(C(\mathrm{SL}(2, \mathbb{R})), \omega, \bar{g})$ is Sasaki, and, in accord with Theorem D.12, it is a Kähler manifold.

It can be verified that the vector $\xi=L^{3}$ is a Killing vector for the metric $(4.20)$, and $\operatorname{SL}(2, \mathbb{R})$ has a K-contact structure, in the sense of Definition D.10. In fact, with Remark A.3, it is verified that $\frac{\partial}{\partial x}$ and $\frac{\partial}{\partial \theta}$ are Killing vectors for the metric (4.21) below, because none of the coordinates $x$ and $\theta$ appear explicitly in (4.20). For completness, if $X=X^{1} \frac{\partial}{\partial x}+X^{2} \frac{\partial}{\partial y}+X^{3} \frac{\partial}{\partial \theta}$ then the equations (A.6) of the Killing vectors in the case of the homogeneous metric (4.21) are

$$
\begin{aligned}
& -2(\alpha+\beta) X^{2}+2(\alpha+\beta) y \partial_{x} X^{1}+4 \beta y^{2} \partial_{x} X^{3}=0, \\
& \alpha \partial_{x} X^{2}+(\alpha+\beta) \partial_{y} X^{1}+2 \beta y \partial_{y} X^{3}=0, \\
& -2 \beta X^{2}+2 \beta y \partial_{x} X^{1}+(\alpha+\beta) \partial_{\theta} X^{1}+2 \beta y \partial_{\theta} X^{3}=0 \\
& -X^{2}+y \partial_{y} X^{2}=0 \\
& 2 \beta y \partial_{y} X^{1}+4 \beta y^{2} \partial_{y} X^{3}+\alpha \partial_{\theta} X^{2}=0 \\
& \beta \partial_{\theta} X^{1}+2 \beta y \partial_{\theta} X^{3}+\partial_{\theta} X^{1}+2 \beta y \partial_{\theta} X^{3}=0 .
\end{aligned}
$$

In fact, we have

Proposition 4.1. The metric on the group $\mathrm{SL}(2, \mathbb{R})$, invariant to the action (4.7), is

$$
\begin{aligned}
\mathrm{d} s_{\mathrm{SL}(2, \mathbb{R})}^{2}(x, y, \theta) & =\lambda_{1}^{2}+\lambda_{2}^{2}+\lambda_{3}^{2}=\alpha \frac{\mathrm{d} x^{2}+\mathrm{d} y^{2}}{y^{2}}+\beta\left(\frac{\mathrm{d} x}{y}+2 \mathrm{~d} \theta\right)^{2} \\
& =\frac{(\alpha+\beta) \mathrm{d} x^{2}+\alpha \mathrm{d} y^{2}}{y^{2}}+4 \beta \mathrm{d} \theta^{2}+4 \frac{\beta}{y} \mathrm{~d} x \mathrm{~d} \theta .
\end{aligned}
$$

The matrix associated with the metric (4.20) is

$$
\begin{aligned}
& g_{\mathrm{SL}(2, \mathbb{R})}(x, y, \theta)=\left(\begin{array}{ccc}
g_{x x} & 0 & g_{x \theta} \\
0 & g_{y y} & 0 \\
g_{\theta x} & 0 & g_{\theta \theta}
\end{array}\right), \\
& g_{x x}=\frac{\alpha+\beta}{y^{2}}, \quad g_{y y}=\frac{\alpha}{y^{2}}, \quad g_{\theta \theta}=4 \beta, \quad g_{x \theta}=\frac{2 \beta}{y} .
\end{aligned}
$$

The invariant vector fields $L^{1}, L^{2}, L^{3}$ given by (4.11) are orthonormal with respect to the metric (4.20). The Killing vector fields associated to the metric (4.20), solutions of the equations (4.19), are given by (4.14).

$\left(L^{3}, \lambda_{3}, \Phi^{\prime}\right)$ defines an almost contact structure on $\mathrm{SL}(2, \mathbb{R})$, where $L^{3}, \lambda_{3}$, $\Phi^{\prime}$ are given respectively by (4.11c), (4.10c), (4.16). $\lambda_{3}$ is the contact structure for $\mathrm{SL}(2, \mathbb{R}), L^{3}$ is the Reeb vector and the contact distribution $\mathcal{D}$ is given by $(4.17) .\left(\mathrm{SL}(2, \mathbb{R})(x, y, \theta), \mathcal{X}_{1}, \mathrm{~d} s_{\mathcal{D}}^{2}\right)$ is a subRiemannian manifold and

$$
\mathrm{d} s_{\mathrm{SL}(2, \mathbb{R})}^{2}(x, y, \theta)=\mathrm{d} s_{\mathcal{X}_{1}}^{2}+\lambda_{3}^{2},
$$


where $\mathrm{d} s_{\mathcal{X}_{1}}^{2}$ is the (Beltrami) Kähler metric (A.11), (A.17),

$$
\mathrm{d} s_{\mathcal{X}_{1}}^{2}=\lambda_{1}^{2}+\lambda_{2}^{2}=\alpha \frac{\mathrm{d} x^{2}+\mathrm{d} y^{2}}{y^{2}}
$$

on the Siegel upper half-plane $x, y, \in \mathbb{R}, y>0$. The invariant vector fields $l_{0}^{1}, l_{0}^{2}$ given by (4.12) are orthonormal with respect to the metric (4.22).

The manifold $\mathrm{SL}(2, \mathbb{R})$ admits the homogenous contact metric structure $\left(\lambda_{3}, L^{3}, \Phi^{\prime}, g_{\mathrm{SL}(2, \mathbb{R})}\right)$. The group $\mathrm{SL}(2, \mathbb{R})$ has the $K$-contact structure associated with $\xi=L^{3}$, and it is a Sasaki manifold with the Riemann cone $(C(\mathrm{SL}(2, \mathbb{R})), \omega, \bar{g})$ with respect to the metric (4.21), where $\bar{g}$ and $\omega$ are given by (4.18).

The last assertion in Proposition (4.1) is well known, see [2, Example 7].

Now we enumerate other invariant metrics on $\operatorname{SL}(2, \mathbb{R})$ appearing in literature, different of the metric (4.20) in Proposition 4.1:

Comment 4.2. An explicit invariant metric on $\mathrm{SL}(2, \mathbb{R})$ in coordinates different of the coordinates $(x, y, \theta)$ appears in [74, Theorem 2, p. 141], see also Theorem A.11. A different form of the invariant metric on $\mathrm{SL}(2, \mathbb{R})$ appears in the context of the BCV spaces, see our Remark A.17, where we have applied the Cayley transform to the metric appearing in Theorem A.15, reproduced after [101] and [47, Example 2.1.10, p. 59]. See also [91, Proposition 2.2, p. 1072 and equation (2.14)].

We also give a direct proof of the some well-known facts, see (b2) in Theorem A.11.

Remark 4.3. The Siegel upper half-plane $\mathcal{X}_{1}$ admits a realization as noncompact Hermitian symmetric space

$$
\mathcal{X}_{1}=\frac{\mathrm{SL}(2, \mathbb{R})}{\mathrm{SO}(2)} \approx \frac{\mathrm{SU}(1,1)}{\mathrm{U}(1)}
$$

$\mathcal{X}_{1}$ is a symmetric, naturally reductive space.

Proof. We use the equivalence (C.14), but we look at the level of groups. We consider the case of $\operatorname{Sp}(n, \mathbb{R})$. The group $\operatorname{Sp}(n, \mathbb{K})$ is the group of matrices $M \in \mathrm{M}(2 n, \mathbb{K})$, where $\mathbb{K}$ is $\mathbb{R}$ or $\mathbb{C}$, for which

$$
\langle M \alpha, M \beta\rangle=\langle\alpha, \beta\rangle, \quad \text { where } \quad \alpha, \beta \in M(n, 1, \mathbb{K}),
$$

and

$$
\langle\alpha, \beta\rangle:=\alpha^{t} J \beta, \quad J=\left(\begin{array}{cc}
0 & \mathbb{1}_{n} \\
-\mathbb{1}_{n} & 0
\end{array}\right),
$$

i.e., we have (4.24)

$$
\operatorname{Sp}(n, \mathbb{R})=\left\{M=\left(\begin{array}{ll}
a & b \\
c & d
\end{array}\right) \in \mathrm{GL}(2 n, \mathbb{R}) \mid M^{t} J M=J\right\}, \quad a, b, c, d \in M(n, \mathbb{R}) .
$$

We can identify the complex linear group $\mathrm{GL}(n, \mathbb{C})$ with the subgroup of matrices of $\mathrm{GL}(2 n, \mathbb{R})$ that commutes with $J$, i.e., $a+\mathrm{i} b \in \mathrm{GL}(n, \mathbb{C})$ is identified with the real $2 n \times 2 n$ matrix (4.25), see, e.g., [71, p. 115]

$$
\left(\begin{array}{cc}
a & b \\
-b & a
\end{array}\right)
$$


It is easy to prove, see, e.g., [97, 60], that if $M \in \operatorname{Sp}(n, \mathbb{R})$, then $M$ is similar with $M^{t}$ and $M^{-1}$. If $M \in \operatorname{Sp}(n, \mathbb{R})$ is as in (4.24), then the matrices $a, b, c, d \in M(n, \mathbb{R})$ in (4.24) verify the equivalent conditions

$$
\begin{array}{lll}
a b^{t}-b a^{t}=0, & a d^{t}-b c^{t}=\mathbb{1}_{n}, & c d^{t}-d c^{t}=0, \\
a^{t} c-c^{t} a=0, & a^{t} d-c^{t} b=\mathbb{1}_{n}, & b^{t} d-d^{t} b=0 .
\end{array}
$$

Note that the inverse of the matrix (4.24) is given by

$$
M^{-1}=\left(\begin{array}{cc}
d^{t} & -b^{t} \\
-c^{t} & a^{t}
\end{array}\right)
$$

The matrices from $\operatorname{Sp}(n, \mathbb{R})$ have the determinant 1 .

Using the expression (4.27) it can be shown that the matrix

$$
M \in \operatorname{Sp}(n, \mathbb{R}) \cap \mathrm{O}_{2 n}
$$

has the expression (4.24) and

$$
M=\left(\begin{array}{cc}
a & b \\
-b & a
\end{array}\right), \quad a^{t} a+b^{t} b=\mathbb{1}_{n}, \quad a^{t} b=b^{t} a .
$$

If $M \in M(2 n, \mathbb{R})$ has the property (4.28), let

$$
M^{\prime}:=a+\mathrm{i} b \in M(n, \mathbb{C}),
$$

and the correspondence $M \rightarrow M^{\prime}$ of (4.28) with (4.29) is a group isomorphism

$$
\mathrm{Sp}(n, \mathbb{R}) \cap \mathrm{O}_{2 n} \approx \mathrm{U}(n) .
$$

We identify $\mathbb{R}^{2 n}$ with $\mathbb{C}^{n}$ via the correspondence $\alpha=(p, q) \mapsto a$,

$$
a=\frac{p+\mathrm{i} q}{\sqrt{2}}, \quad p, q \in \mathbb{R}^{n},
$$

Following Bargmann [7], it is useful to introduce the transformation

$$
\begin{aligned}
& \mathcal{W}: \mathbb{R}^{2 n} \leftrightarrow \mathbb{C}^{2 n}, \quad \alpha=(p, q) \mapsto \alpha_{\mathbb{C}}=(a, \bar{a}), \\
& \alpha_{\mathbb{C}}=\mathcal{W} \alpha, \quad \mathcal{W}=2^{-\frac{1}{2}}\left(\begin{array}{cc}
\mathbb{1}_{n} & \mathrm{i} \mathbb{1}_{n} \\
\mathbb{1}_{n} & -\mathrm{i} \mathbb{1}_{n}
\end{array}\right), \quad \mathcal{W}^{-1}=2^{-\frac{1}{2}}\left(\begin{array}{cc}
\mathbb{1}_{n} & \mathbb{1}_{n} \\
-\mathrm{i} \mathbb{1}_{n} & \mathrm{i} \mathbb{1}_{n}
\end{array}\right),
\end{aligned}
$$

where

$$
\alpha^{t}=\left(p^{t}, q^{t}\right), \quad p^{t}=\left(p_{1}, \ldots, p_{n}\right) .
$$

To $M \in M(2 n, \mathbb{R})$ as in $(4.24)$ we associate $M_{\mathbb{C}} \in M(2 n, \mathbb{C})$

$$
M_{\mathbb{C}}=\mathcal{W} M \mathcal{W}^{-1}=\frac{1}{2}\left(\begin{array}{ll}
a+d+\mathrm{i}(c-b) & a-d+\mathrm{i}(b+c) \\
a-d-\mathrm{i}(c+b) & a+d+\mathrm{i}(b-c)
\end{array}\right) .
$$

If $\beta=R \alpha, R \in \operatorname{Sp}(n, \mathbb{R})$ then

$$
\beta_{\mathbb{C}}=R_{\mathbb{C}} \alpha_{\mathbb{C}}, \quad \text { where } \quad R_{\mathbb{C}}=\mathcal{W} R \mathcal{W}^{-1} \in \operatorname{Sp}(n, \mathbb{R})_{\mathbb{C}},
$$

and $R_{\mathbb{C}} \in \operatorname{Sp}(n, \mathbb{R})_{\mathbb{C}}=\operatorname{Sp}(n, \mathbb{C}) \cap \mathrm{U}(n, n)$, cf. $[7,59,66]$. 
From (4.26) we see that $\operatorname{SL}(2, \mathbb{R}) \approx \operatorname{Sp}(1, \mathbb{R})$. Next we apply (4.30) to $M=\left(\begin{array}{ll}a & b \\ c & d\end{array}\right) \in \operatorname{SL}(2, \mathbb{R})$,

and we get $M_{\mathbb{C}}=\left(\begin{array}{cc}\alpha & \beta \\ \bar{\beta} & \bar{\alpha}\end{array}\right)$, where $\alpha=\frac{1}{2}(a+d+\mathrm{i}(c-b)), \beta=\frac{1}{2}(a-d+\mathrm{i}(b+c))$, i.e., $M_{\mathbb{C}} \in \mathrm{SU}(1,1)$ because $|\alpha|^{2}-|\beta|^{2}=1$. In particular, if $a=\cos \theta, b=\sin \theta, c=-b, d=a$, then $\alpha=\mathrm{e}^{-\mathrm{i} \theta}$, $\beta=0$, and (4.23) is proved.

Now we prove that $\mathcal{X}_{1}$ is a naturally reductive space verifying that the condition (A.10) is fulfilled. We take into account (C.14) and (C.15a). If we consider $\mathfrak{s l}(2, \mathbb{R}) \ni \mathfrak{g}=\mathfrak{m}+\mathfrak{h}$ for the homogeneous space $M=\mathcal{X}_{1}$ as in (4.23), then $\mathfrak{h}=\langle H\rangle, \mathfrak{m}=\langle F, G\rangle=\langle F-G, F+G\rangle$, and the relation $(\mathrm{A} .7 \mathrm{c})[\mathfrak{m}, \mathfrak{h}] \subset \mathfrak{m}$ follows, i.e., $\mathcal{X}_{1}$ is reductive.

In order to verify the condition (A.10), we take $X=a L^{1}+b L^{3}, Y=a_{1} L^{1}+b_{1} L^{3}, Z=$ $a_{2} L^{1}+b_{2} L^{3}$. We get $[X, Z]=\left(a_{2} b-b_{2} a\right) L^{2} \in \mathfrak{h}$ and $[X, Z]_{\mathfrak{m}}=0$. (A.10) is trivially satisfied.

We mention that naturally reductive left-invariant metrics on $\operatorname{SL}(2, \mathbb{R})$ in the context of BCV-spaces have been investigated in [62].

\section{$5 \quad$ The Jacobi group $G_{1}^{J}(\mathbb{R})$ embedded in $\operatorname{Sp}(2, \mathbb{R})$}

\subsection{The composition law}

The real Jacobi group $G_{1}^{J}(\mathbb{R})$ is the semi-direct product of the real three dimensional Heisenberg group $H_{1}$ with $\mathrm{SL}(2, \mathbb{R})$. The Lie algebra of the Jacobi group $G_{1}^{J}(\mathbb{R})$ is given by $\mathfrak{g}_{1}^{J}(\mathbb{R})=$ $\langle P, Q, R, F, G, H\rangle_{\mathbb{R}}$, where the first three generators $P, Q, R$ of $\mathfrak{h}_{1}$ verify the commutation relations (3.2), the generators $F, G, H$ of $\mathfrak{s l}(2, \mathbb{R})$ verify the commutation relations (C.11) and the ideal $\mathfrak{h}_{1}$ in $\mathfrak{s p}(2, \mathbb{R})$ is determined by the non-zero commutation relations

$$
[P, F]=Q, \quad[Q, G]=P, \quad[P, H]=P, \quad[H, Q]=Q .
$$

Let $G_{1}^{J}(\mathbb{R}) \ni g=(M, h)$, where $M$ is as in (4.1), while $H_{1} \ni h=(X, \kappa), X=(\lambda, \mu) \in \mathbb{R}^{2}$ and similarly for $g^{\prime}=\left(M^{\prime}, h^{\prime}\right)$. The composition law of $G_{1}^{J}(\mathbb{R})$ is

$$
g g^{\prime}=g_{1}, \quad \text { where } \quad M_{1}=M M^{\prime}, \quad X_{1}=X M^{\prime}+X^{\prime}, \quad \kappa_{1}=\kappa+\kappa^{\prime}+\left|\begin{array}{c}
X M^{\prime} \\
X^{\prime}
\end{array}\right|
$$

where

$$
\begin{aligned}
& g_{1}=\left(\begin{array}{ll}
a a^{\prime}+b c^{\prime} & a b^{\prime}+b d^{\prime} \\
c a^{\prime}+d c^{\prime} & c b^{\prime}+d d^{\prime}
\end{array}\right), \\
& \left(\lambda_{1}, \mu_{1}\right)=\left(\lambda^{\prime}+\lambda a^{\prime}+\mu c^{\prime}, \mu^{\prime}+\lambda b^{\prime}+\mu d^{\prime}\right), \quad \kappa_{1}=\kappa+\kappa^{\prime}+\lambda q^{\prime}-\mu p^{\prime} .
\end{aligned}
$$

The inverse element of $g \in G_{1}^{J}(\mathbb{R})$ is given by

$$
(M, X, \kappa)^{-1}=\left(M^{-1},-Y,-\kappa\right) \rightarrow g^{-1}=\left(\begin{array}{cccc}
d & 0 & -b & -\mu \\
-p & 1 & -q & -\kappa \\
-c & 0 & a & \lambda \\
0 & 0 & 0 & 1
\end{array}\right)
$$

where $Y$ was defined in (1.5).

Using the notation of [39, p. 9], the EZ-coordinates (EZ - from Eichler and Zagier) of an element $g \in G_{1}^{J}(\mathbb{R})(1.4)$ are $(x, y, \theta, \lambda, \mu, \kappa)$, where $M$ is related with $(x, y, \theta)$ by (4.4), (4.5).

The $S$-coordinates ( $\mathrm{S}$ - from Siegel) of $g=(M, h) \in G_{1}^{J}(\mathbb{R})$ are $(x, y, \theta, p, q, \kappa)$, where $(x, y, \theta)$ are expressed as function of $M \in \mathrm{SL}(2, \mathbb{R})$ by $(4.5)$. 


\subsection{The action}

Let

$$
\tau:=x+\mathrm{i} y, \quad z:=p \tau+q=\xi+\mathrm{i} \eta, \quad \xi, \eta \in \mathbb{R} .
$$

Let $\mathcal{X}_{1}^{J} \approx \mathcal{X}_{1} \times \mathbb{R}^{2}$ be the Siegel-Jacobi upper half-plane, where $\mathcal{X}_{1}=\{\tau \in \mathbb{C}, y:=\operatorname{Im} \tau>0\}$ is the Siegel upper half-plane, and $\tilde{\mathcal{X}}_{1}^{J}=\mathcal{X}_{1}^{J} \times \mathbb{R}$ denotes the extended Siegel-Jacobi upper half-plane. Simultaneously with the Jacobi group $G_{1}^{J}(\mathbb{R})$ consisting of elements $(M, X, \kappa)$, we consider the group $G^{J}(\mathbb{R})_{0}$ of elements $(M, X)$. It should be mentioned that there is a group homomorphism $G_{1}^{J}(\mathbb{R}) \ni(M, X, \kappa) \mapsto(M, X) \in G^{J}(\mathbb{R})_{0}$, through which the action of $G_{1}^{J}(\mathbb{R})$ on $\mathcal{X}_{1}^{J}$ can be defined, see [17, Proposition 2]. Then

Lemma 5.1. The action $G^{J}(\mathbb{R})_{0} \times \mathcal{X}_{1}^{J} \rightarrow \mathcal{X}_{1}^{J}$ is given by

$$
\begin{aligned}
& (M, X) \times\left(\tau^{\prime}, z^{\prime}\right)=\left(\tau_{1}, z_{1}\right), \quad \text { where } \quad \tau_{1}=\frac{a \tau^{\prime}+b}{c \tau^{\prime}+d}, \quad z_{1}=\frac{z^{\prime}+\lambda \tau^{\prime}+\mu}{c \tau^{\prime}+d}, \\
& (M, X) \times\left(x^{\prime}, y^{\prime}, p^{\prime}, q^{\prime}\right)=\left(x_{1}, y_{1}, p_{1}, q_{1}\right),
\end{aligned}
$$

where $z^{\prime}=p^{\prime} \tau^{\prime}+q^{\prime}, \tau^{\prime}=x^{\prime}+\mathrm{i} y^{\prime}$ as in (5.4),

$$
\left(p_{1}, q_{1}\right)=(p, q)+\left(p^{\prime}, q^{\prime}\right)\left(\begin{array}{ll}
a & b \\
c & d
\end{array}\right)^{-1}=\left(p+d p^{\prime}-c q^{\prime}, q-b p^{\prime}+a q^{\prime}\right)
$$

while $\left(x_{1}, y_{1}\right)$ are given by $(4.7 \mathrm{a})$.

The action $G_{1}^{J}(\mathbb{R}) \times \tilde{\mathcal{X}}_{1}^{J} \rightarrow \tilde{\mathcal{X}}_{1}^{J}$ is given by

$$
\begin{aligned}
& (M, X, \kappa) \times\left(\tau^{\prime}, z^{\prime}, \kappa^{\prime}\right)=\left(\tau_{1}, z_{1}, \kappa_{1}\right), \\
& (M, X, \kappa) \times\left(x^{\prime}, y^{\prime}, p^{\prime}, q^{\prime}, \kappa^{\prime}\right)=\left(x_{1}, y_{1}, p_{1}, q_{1}, \kappa_{1}\right), \\
& \kappa_{1}=\kappa+\kappa^{\prime}+\lambda q^{\prime}-\mu p^{\prime}, \quad\left(p^{\prime}, q^{\prime}\right)=\left(\frac{\eta^{\prime}}{y^{\prime}}, \xi^{\prime}-\frac{x^{\prime}}{y^{\prime}} \eta^{\prime}\right) .
\end{aligned}
$$

Proof. The assertion (5.5) is expressed in [39, p. 11], reproduced in [13, Remark 9.1]. Details of the proof are given in [14, Remark 1]. The calculation of $\kappa_{1}$ in (5.8) is an easy consequence of the composition law (5.2). The expression of $\left(p^{\prime}, q^{\prime}\right)$ in (5.8) is a consequence of (5.4).

\subsection{Fundamental vector fields}

In order to calculate the change of coordinates of a contravariant vector field under the change of variables $(5.4)(x, y, \xi, \eta) \rightarrow(x, y, p, q)$, where $(p, q)=\left(\frac{\eta}{y}, \xi-\frac{\eta}{y} x\right)$, we firstly observe that the Jacobian is $\frac{\partial(x, y, \xi, \eta)}{\partial(x, y, p, q)}=-y<0$, and we get easily

$$
\partial_{x} \rightarrow \partial_{x}-p \partial_{q}, \quad \partial_{y} \rightarrow \partial_{y}-\frac{p}{y}\left(\partial_{p}-x \partial_{q}\right), \quad \partial_{\xi} \rightarrow \partial_{q}, \quad \partial_{\eta} \rightarrow \frac{1}{y}\left(\partial_{p}-x \partial_{q}\right) .
$$

In order to calculate the change of coordinates of a contravariant vector field under the change of variables $(5.4)(x, y, p, q, \kappa) \rightarrow(x, y, \xi, \eta, \kappa)$, we get easily

$$
\partial_{x} \rightarrow \partial_{x}+p \partial_{\xi}, \quad \partial_{y} \rightarrow \partial_{y}+p \partial_{\eta}, \quad \partial_{p} \rightarrow x \partial_{\xi}+y \partial_{\eta}, \quad \partial_{q} \rightarrow \partial_{\xi}
$$

With (5.9) and the action (5.5) on $\mathcal{X}_{1}^{J}$, and then with (5.10) for the action (5.8) on $\hat{\mathcal{X}}_{1}^{J}$, we get 
Proposition 5.2. The fundamental vector fields expressed in coordinates $(\tau, z)$ of the SiegelJacobi upper half-plane $\mathcal{X}_{1}^{J}$ on which act the reduced Jacobi group $G^{J}(\mathbb{R})_{0}$ by (5.5) are given by the holomorphic vector fields

$$
\begin{aligned}
& F^{*}=\partial_{\tau}, \quad G^{*}=-\tau^{2} \partial_{\tau}-z \tau \partial_{z}, \quad H^{*}=2 \tau \partial_{\tau}+z \partial_{z}, \\
& P^{*}=\tau \partial_{z}, \quad Q^{*}=\partial_{z}, \quad R^{*}=0 .
\end{aligned}
$$

Then the real holomorphic fundamental vector fields corresponding to $\tau=x+\mathrm{i} y, y>0$, $z=\xi+\mathrm{i} \eta$ in the variables $(x, y, \xi, \eta)$ are

$$
\begin{aligned}
& F^{*}=F_{1}^{*}, \quad G^{*}=G_{1}^{*}+(\eta y-\xi x) \partial_{\xi}-(\xi y+x \eta) \partial_{\eta}, \\
& H^{*}=H_{1}^{*}+\xi \partial_{\xi}+\eta \partial_{\eta}, \quad P^{*}=x \partial_{\xi}+y \partial_{\eta}, \quad Q^{*}=\partial_{\xi}, \quad R^{*}=0,
\end{aligned}
$$

where $F_{1}^{*}, G_{1}^{*}, H_{1}^{*}$ are the fundamental vector fields (C.23) attached to the generators $F, G, H$ of $\mathfrak{s l}(2, R)$ corresponding to the action $(5.5)$ of $\mathrm{SL}(2, \mathbb{R})$ on $\mathcal{X}_{1}$.

If we express the fundamental vector fields in the variables $(x, y, p, q)$ where $\xi=p x+q$, $\eta=p y$, we find

$$
\begin{aligned}
& F^{*}=F_{1}^{*}-p \partial_{q}, \quad G^{*}=G_{1}^{*}-q \partial_{p}, \quad H^{*}=H_{1}^{*}-p \partial_{p}+q \partial_{q}, \\
& P^{*}=\partial_{p}, \quad Q^{*}=\partial_{q}, \quad R^{*}=0 .
\end{aligned}
$$

Now we consider the action $(5.8)$ of $G_{1}^{J}(\mathbb{R})$ on the points $(\tau, z, \kappa)$ of $\tilde{\mathcal{X}}_{1}^{J}$.

Instead of (5.11), we get the fundamental vector fields in the variables $(\tau, z, p, q, \kappa)$

$$
\begin{aligned}
& F^{*}=\partial_{\tau}, \quad G^{*}=-\tau^{2} \partial_{\tau}-z \tau \partial_{z}, \quad H^{*}=2 \tau \partial_{\tau}+z \partial_{z}, \\
& P^{*}=\tau \partial_{z}+q \partial_{\kappa}, \quad Q^{*}=\partial_{z}-p \partial_{\kappa}, \quad R^{*}=\partial_{\kappa}, \quad p=\frac{\operatorname{Im}(z)}{\operatorname{Im}(\tau)}, \quad q=\frac{\operatorname{Im}(\bar{z} \tau)}{\operatorname{Im}(\tau)} .
\end{aligned}
$$

Instead of (5.12), we get the fundamental vector fields in $\tilde{\mathcal{X}}_{1}^{J}$ in the variables $(x, y, \xi, \eta, \kappa)$

$$
\begin{aligned}
& F^{*}=F_{1}^{*}, \quad G^{*}=G_{1}^{*}+(\eta y-\xi x) \partial_{\xi}-(\xi y+x \eta) \partial_{\eta}, \\
& H^{*}=H_{1}^{*}+\xi \partial_{\xi}+\eta \partial_{\eta}, \quad P^{*}=x \partial_{\xi}+y \partial_{\eta}+q \partial_{\kappa}, \quad Q^{*}=\partial_{\xi}-p \partial_{\kappa}, \quad R^{*}=\partial_{\kappa} .
\end{aligned}
$$

Instead of (5.13), we ge the fundamental vector fields in the variables $(x, y, p, q, \kappa)$

$$
\begin{aligned}
& F^{*}=F_{1}^{*}-p \partial_{q}, \quad G^{*}=G_{1}^{*}-q \partial_{p}, \quad H^{*}=H_{1}^{*}-p \partial_{p}+q \partial_{q}, \\
& P^{*}=\partial_{p}+q \partial_{k}, \quad Q^{*}=\partial_{q}-p \partial_{k}, \quad R^{*}=\partial_{\kappa} .
\end{aligned}
$$

\subsection{Invariant metrics}

We explain the method to get invariant metrics on $G$-homogeneous manifolds $M$ from invariant metrics of $G$, see also Appendix D.1.1.

Let $M=G / H$ be a reductive homogeneous space. If $\mathfrak{g}(\mathfrak{h})$ is the Lie algebra of $G$ (respectively, $H$ ), then there exists a vector space $\mathfrak{m}$ such that we have the vector space decomposition $\mathfrak{g}=\mathfrak{m}+\mathfrak{h}, \mathfrak{m} \cap \mathfrak{h}=\varnothing$, and the tangent space at $x, T_{x} M$, can be identified with $\mathfrak{m}$, where $H=G_{x}$ is the isotropy group at $x$, see Definition A.5 and Lemma A.6. Then let $X_{i}, i=1, \ldots, n$ be a basis of the Lie algebra $\mathfrak{g}$ such that

$$
\mathfrak{m}=\left\langle X_{1}, \ldots, X_{m}\right\rangle, \quad \mathfrak{h}=\left\langle X_{m+1}, \ldots, X_{n}\right\rangle,
$$

where $\operatorname{dim} \mathfrak{m}=m$. The left-invariant one forms $\lambda_{i}$ on $G$ are given by

$$
g^{-1} \mathrm{~d} g=\sum_{i=1}^{n} \lambda_{i} X_{i},
$$


and the left-invariant vector fields $L^{i}$ on $G$ are determined from the relations $\left\langle\lambda_{i} \mid L^{j}\right\rangle=\delta_{i j}$, $i, j=1, \ldots, n$. Then the invariant metric on $G$ is given by $\mathrm{d} s_{G}^{2}=\sum_{i=1}^{n} \lambda_{i}^{2}$ and $g_{G}\left(L^{i}, L^{j}\right)=\delta_{i, j}$, where $i, j=1, \ldots, n$. Let now $L_{0}^{j}$ be the projections on $M$ of the vector fields $L^{j}, j=1, \ldots, m$. We stil have $\left\langle\lambda_{i} \mid L_{0}^{j}\right\rangle=\delta_{i j}, i, j=1, \ldots, m$ and $\mathrm{d} s_{M}^{2}=\sum_{i=1}^{m} \lambda_{i}^{2}$. The fundamental vector fields $X_{i}^{*}, i=1, \ldots, m$ are Killing vectors of the metric $g_{M}$.

Now we calculate the left-invariant one-forms on $G_{1}^{J}(\mathbb{R})$

$$
g^{-1} \mathrm{~d} g=\lambda^{F} F+\lambda^{G} G+\lambda^{H} H+\lambda^{P} P+\lambda^{Q} Q+\lambda^{R} R,
$$

where $g$ is as in (1.4) and $g^{-1}$ as in (5.3). We find the left-invariant one-forms on $G_{1}^{J}(\mathbb{R})$

$$
\begin{aligned}
\lambda^{F} & =\lambda^{f}, \quad \lambda^{G}=\lambda^{g}, \quad \lambda^{H}=\lambda^{h}, \\
\lambda^{P} & =\mathrm{d} \lambda-p \mathrm{~d} a-q \mathrm{~d} c=c \mathrm{~d} q+a \mathrm{~d} p=\lambda^{p}-\lambda \lambda^{h}-\mu \lambda^{g} \\
& =-y^{-\frac{1}{2}} \sin \theta \mathrm{d} q+\left(y^{\frac{1}{2}} \cos \theta-x y^{-\frac{1}{2}} \sin \theta\right) \mathrm{d} p, \\
\lambda^{Q} & =d \mathrm{~d} q+b \mathrm{~d} p=\lambda^{q}-p \mathrm{~d} b-q \mathrm{~d} d=\lambda^{q}-\lambda \lambda^{f}+\mu \lambda^{h} \\
& =y^{-\frac{1}{2}} \cos \theta \mathrm{d} q+\left(y^{\frac{1}{2}} \sin \theta+x y^{-\frac{1}{2}} \cos \theta\right) \mathrm{d} p, \\
\lambda^{R} & =\mathrm{d} \kappa-p \mathrm{~d} q+q \mathrm{~d} p=\lambda^{r}+\lambda^{2} \lambda^{f}-\mu^{2} \lambda^{g}-2 \lambda \mu \lambda^{h} .
\end{aligned}
$$

In (5.15), equations $(5.15 \mathrm{c}),(5.15 \mathrm{e}),(5.15 \mathrm{f})$ are expressed in the S-coordinates $(x, y, \theta, p, q, \kappa)$, $\lambda^{p}, \lambda^{q}, \lambda^{r}$ have the expression (3.3) in the $(\lambda, \mu, \kappa)$-coordinates, while $\lambda^{f}, \lambda^{g}, \lambda^{h}$ have the expressions (4.2) in the $(a, b, c, d)$-coordinates of $\operatorname{SL}(2, \mathbb{R})$ and (4.8) are expressed in the $(x, y, \theta)$ coordinates. Also the elements $a, b, c, d$ of the matrix $M \in \mathrm{SL}(2, \mathbb{R})(1.4)$ are expressed in the $(x, y, \theta)$-coordinates by $(4.4)$.

Now we calculate the left-invariant vector fields for the real Jacobi group

Proposition 5.3. The left-invariant vector fields $L^{\alpha}$ for the real Jacobi group $G_{1}^{J}(\mathbb{R})$ orthogonal with respect to the invariant one-forms $\lambda^{\beta}$,

$$
\left\langle\lambda^{\beta} \mid L^{\alpha}\right\rangle=\delta_{\alpha, \beta}, \quad \alpha, \beta=F, G, H, P, Q, R,
$$

are given by the equations

$$
\begin{aligned}
& L^{F}=L^{f}, \quad L^{G}=L^{g}, \quad L^{H}=L^{h}, \quad L^{P}=L_{0}^{P}+L_{+}^{P}, \quad L^{Q}=L_{0}^{Q}+L_{+}^{Q}, \\
& L_{0}^{P}=d \frac{\partial}{\partial p}-b \frac{\partial}{\partial q}=\frac{\cos \theta}{y^{\frac{1}{2}}} \frac{\partial}{\partial p}-\frac{x \cos \theta+y \sin \theta}{y^{\frac{1}{2}}} \frac{\partial}{\partial q}, \\
& L_{+}^{P}=-(p b+q d) \frac{\partial}{\partial \kappa}=-\frac{1}{y^{1 / 2}}[p(x \cos \theta+y \sin \theta)+q \cos \theta] \frac{\partial}{\partial \kappa}, \\
& L_{0}^{Q}=-c \frac{\partial}{\partial p}+a \frac{\partial}{\partial q}=\frac{\sin \theta}{y^{\frac{1}{2}}} \frac{\partial}{\partial p}+\frac{y \cos \theta-x \sin \theta}{y^{\frac{1}{2}}} \frac{\partial}{\partial q}, \\
& L_{+}^{Q}=(p a+q c) \frac{\partial}{\partial \kappa}=\frac{1}{y^{1 / 2}}[p(y \cos \theta-x \sin \theta)-q \sin \theta] \frac{\partial}{\partial \kappa}, \\
& L^{R}=\frac{\partial}{\partial \kappa} .
\end{aligned}
$$

The invariant vector fields $L^{F}, L^{G}, L^{H}, L^{P}, L^{Q}, L^{R}$ verify the commutations relations (C.11), (3.2) and (5.1) of the generators $F, G, H, P, Q, R$ of the Lie algebra $\mathfrak{g}_{1}^{J}(\mathbb{R})$.

Besides the formulas for $\lambda_{1}, \lambda_{2}, \lambda_{3}$ defined in (4.10), we introduce the left-invariant one-forms:

$$
\lambda_{4}=\sqrt{\gamma} \lambda^{P}, \lambda_{5}=\sqrt{\gamma} \lambda^{Q}, \quad \lambda_{6}=\sqrt{\delta} \lambda^{R}, \quad \gamma, \delta>0,
$$


where $\lambda^{P}, \lambda^{Q}, \lambda^{R}$ are defined in (5.15). Note that the parameters $\gamma, \delta$ introduced in (5.17) will appear also in the invariant metrics (5.25) on $\tilde{\mathcal{X}}_{1}^{J}$ and (5.27) on $G_{1}^{J}(\mathbb{R})$, while in the metric (5.22) on $\mathcal{X}_{1}^{J}$ appears only $\gamma$. The invariant metrics on $\mathcal{D}_{n}^{J}$ and $\mathcal{X}_{n}^{J}$ depend only of two parameters $\alpha, \gamma>0[16,17,21,109,111]$. In fact, the first time they appear in the papers of Kähler [68, 69] and Berndt [37, 38], parameterizing the invariant metric on $\mathcal{X}_{1}^{J}$.

Also, besides the left-invariant vector fields $L^{1}, L^{2}, L^{3}$ defined in (4.11), we introduce the left invariant one forms

$$
L^{4}=\frac{1}{\sqrt{\gamma}} L^{P}, \quad L^{5}=\frac{1}{\sqrt{\gamma}} L^{Q}, \quad L^{6}=\frac{1}{\sqrt{\delta}} L^{R},
$$

where $L^{P}, L^{Q}, L^{R}$ are defined in (5.16). The vector fields $L^{i}, i=1, \ldots, 6$ verify the commutations relations

$$
\begin{array}{llll}
{\left[L^{1}, L^{2}\right]=-\frac{\sqrt{\beta}}{\alpha} L^{3},} & {\left[L^{2}, L^{3}\right]=\frac{1}{2 \sqrt{\beta}} L^{1},} & {\left[L^{3}, L^{1}\right]=\frac{1}{\sqrt{\beta}} L^{2},} \\
{\left[L^{1}, L^{4}\right]=-\frac{1}{2 \sqrt{\alpha}} L^{5},} & {\left[L^{1}, L^{5}\right]=-\frac{1}{2 \sqrt{\alpha}} L^{4},} & {\left[L^{1}, L^{6}\right]=0,} \\
{\left[L^{2}, L^{4}\right]=-\frac{1}{2 \sqrt{\alpha}} L^{4},} & {\left[L^{2}, L^{5}\right]=\frac{1}{2 \sqrt{\alpha}} L^{5},} & {\left[L^{2}, L^{6}\right]=0,} \\
{\left[L^{3}, L^{4}\right]=-\frac{1}{2 \sqrt{\alpha}} L^{5},} & {\left[L^{3}, L^{5}\right]=\frac{1}{2 \sqrt{\beta}} L^{4},} & {\left[L^{3}, L^{6}\right]=0,} \\
{\left[L^{4}, L^{5}\right]=\frac{2 \sqrt{\delta}}{\gamma} L^{6},} & {\left[L^{4}, L^{6}\right]=0,} & & {\left[L^{5}, L^{6}\right]=0 .}
\end{array}
$$

Similarly, we introduce

$$
L_{0}^{4}=\frac{1}{\sqrt{\gamma}} L_{0}^{P}, \quad L_{0}^{5}=\frac{1}{\sqrt{\gamma}} L_{0}^{Q}, \quad L_{0}^{6}=\frac{1}{\sqrt{\delta}} L^{R} .
$$

Recalling also Proposition 2.1, where we have replaced $u=p v+q, v=x+\mathrm{i} y$ with $z=p \tau+q$, respectively $\tau=x+\mathrm{i} y$, and $k=2 c_{1}, \mu=\frac{c_{2}}{2}$, we have proved:

Proposition 5.4. The balanced metric (5.21) on the Siegel-Jacobi upper half-plane $\mathcal{X}_{1}^{J}$, leftinvariant to the action (5.5), (5.6), (5.7) of reduced group $G^{J}(\mathbb{R})_{0}$ is

$$
\begin{aligned}
\mathrm{d} s_{\mathcal{X}_{1}^{J}}^{2}(\tau, z)=-c_{1} & \frac{\mathrm{d} \tau \mathrm{d} \bar{\tau}}{(\tau-\bar{\tau})^{2}}+\frac{2 \mathrm{i} c_{2}}{\tau-\bar{\tau}}(\mathrm{d} z-p \mathrm{~d} \tau) \times c c, \quad p=\frac{z-\bar{z}}{\tau-\bar{\tau}} \\
\mathrm{d} s_{\mathcal{X}_{1}^{J}}^{2}(x, y, p, q)= & c_{1} \frac{\mathrm{d} x^{2}+\mathrm{d} y^{2}}{4 y^{2}}+\frac{c_{2}}{y}\left[\left(x^{2}+y^{2}\right) \mathrm{d} p^{2}+\mathrm{d} q^{2}+2 x \mathrm{~d} p \mathrm{~d} q\right] \\
= & c_{1} \frac{\mathrm{d} x^{2}+\mathrm{d} y^{2}}{4 y^{2}}+c_{2} \frac{x^{2}+y^{2}}{y}\left[\left(\mathrm{~d} p+\frac{x}{x^{2}+y^{2}} \mathrm{~d} q\right)^{2}+\left(\frac{y \mathrm{~d} q}{x^{2}+y^{2}}\right)^{2}\right], \\
\mathrm{d} s_{\mathcal{X}_{1}^{J}}^{2}(x, y, \xi, \eta)= & c_{1} \frac{\mathrm{d} x^{2}+\mathrm{d} y^{2}}{4 y^{2}} \\
& +\frac{c_{2}}{y}\left[\mathrm{~d} \xi^{2}+\mathrm{d} \eta^{2}+\left(\frac{\xi}{y}\right)^{2}\left(\mathrm{~d} x^{2}+\mathrm{d} y^{2}\right)-2 \frac{\eta}{y}(\mathrm{~d} x \mathrm{~d} \xi+\mathrm{d} y \mathrm{~d} \eta)\right]
\end{aligned}
$$

The metric (5.21) is Kähler.

If we denote $\frac{c_{1}}{4}=\alpha, c_{2}=\gamma$, then the matrix attached to the left invariant metric (5.21b) on $\mathcal{X}_{1}^{J}$ reads

$$
g_{\mathcal{X}_{1}^{J}}=\left(\begin{array}{cccc}
g_{x x} & 0 & 0 & 0 \\
0 & g_{y y} & 0 & 0 \\
0 & 0 & g_{p p} & g_{p q} \\
0 & 0 & g_{p q} & g_{q q}
\end{array}\right),
$$




$$
g_{x x}=g_{y y}=\frac{\alpha}{y^{2}}, \quad g_{p p}=\gamma \frac{x^{2}+y^{2}}{y}, \quad g_{q q}=\frac{\gamma}{y}, \quad g_{p q}=\gamma \frac{x}{y} .
$$

The metric (5.21b) can be written as

$$
\mathrm{d} s_{\mathcal{X}_{1}^{J}}^{2}=\lambda_{1}^{2}+\lambda_{2}^{2}+\lambda_{4}^{2}+\lambda_{5}^{2} .
$$

The vector fields $L_{0}^{j}$ dual orthogonal to the invariant one-forms $\lambda_{i},\left\langle\lambda_{i} \mid L_{0}^{j}\right\rangle=\delta_{i j}, i, j=1,2,4,5$, with respect to the bases $\mathrm{d} x, \mathrm{~d} y, \mathrm{~d} p, \mathrm{~d} q$ and $\frac{\partial}{\partial x}, \frac{\partial}{\partial y}, \frac{\partial}{\partial p}, \frac{\partial}{\partial q}$ are

$$
L_{0}^{1}=\frac{y}{\sqrt{\alpha}}\left(\cos 2 \theta \frac{\partial}{\partial x}+\sin 2 \theta \frac{\partial}{\partial y}\right), \quad L_{0}^{2}=\frac{y}{\sqrt{\alpha}}\left(-\sin 2 \theta \frac{\partial}{\partial x}+\cos 2 \theta \frac{\partial}{\partial y}\right),
$$

and $L_{0}^{4}, L_{0}^{5}$ defined by (5.20). The metric (5.21b) is orthonormal with respect to the vector fields $L_{0}^{1}, L_{0}^{2}, L_{0}^{4}, L_{0}^{5}$.

The fundamental vector fields given by (5.13) are the solutions of the equations of the Killing vector fields (5.23) on $\mathcal{X}_{1}^{J}$ in the variables $(x, y, p, q)$ corresponding to the metric $(5.21 \mathrm{~b})$, invariant to the action (5.5), made explicit in (4.7) and (5.7), (5.8):

$$
\begin{aligned}
& -X^{2}+y \partial_{x} X^{1}=0 \\
& \partial_{x} X^{2}+\partial_{y} X^{1}=0 \\
& c_{2}\left[\left(x^{2}+y^{2}\right) \partial_{x} X^{3}+x \partial_{x} X^{4}\right]+\frac{c_{1}}{4 y} \partial_{p} X^{1}=0 \\
& \frac{c_{1}}{4 y} \partial_{q} X^{1}+c_{2}\left(x \partial_{x} X^{3}+\partial_{x} X^{4}\right)=0 \\
& -X^{2}+y \partial_{y} X^{2}=0, \\
& c_{2}\left[\left(x^{2}+y^{2}\right) \partial_{y} X^{3}+\frac{x}{y} \partial_{y} X^{4}\right]+\frac{c_{1}}{y} \partial_{p} X^{2}=0 \\
& c_{2}\left[x \partial_{y} X^{3}+\partial_{y} X^{4}\right]+\frac{c_{1}}{4 y} \partial_{q} X^{2}=0 \\
& 2 x y X^{1}+\left(-x^{2}+y^{2}\right) X^{2}+2 y\left(x^{2}+y^{2}\right) \partial_{p} X^{3}+2 x y \partial_{p} X^{4}=0, \\
& y X^{1}-x X^{2}+x y \partial_{p} X^{3}+y \partial_{p} X^{4}+y\left(x^{2}+y^{2}\right) \partial_{q} X^{3}+x y \partial_{q} X^{4}=0, \\
& -X^{2}+2 x y \partial_{q} X^{3}+2 y \partial_{q} X^{4}=0 .
\end{aligned}
$$

We make a "historical" comment

Comment 5.5. In [37, p. 8], Berndt considered the closed two-form $\Omega=\mathrm{d} \overline{\mathrm{d}} f$ on Siegel-Jacobi upper half-plane $\mathcal{X}_{1}^{J}, G^{J}(\mathbb{R})_{0}$-invariant to the action (5.5), obtained from the Kähler potential

$$
f(\tau, z)=c_{1} \log (\tau-\bar{\tau})-\mathrm{i} c_{2} \frac{(z-\bar{z})^{2}}{\tau-\bar{\tau}}, \quad c_{1}, c_{2}>0,
$$

where $c_{1}=\frac{k}{2}, c_{2}=2 \mu$ comparatively to our formula (2.4). Formula (5.24) is presented by Berndt as "communicated to the author by Kähler", where it is also given equation (5.21a), while (5.21b) has two printing errors. Later, in Section 36 of his last paper [68], reproduced also in [69], Kähler argues how to choose the potential as in (5.24); see also [68, Section 37, equation (9)], where $c_{1}=\frac{\lambda}{2}, c_{2}=\mathrm{i} \mu \pi$, and the metric (8) differs from the metric (5.21) by a factor two, because the hermitian metric used by Kähler is $\mathrm{d} s^{2}=2 g_{i j} \mathrm{~d} z_{i} \mathrm{~d} \bar{z}_{j}$.

We also recall that in [110] Yang calculated the metric on $\mathcal{X}_{n}^{J}$, invariant to the action of $G_{n}^{J}(\mathbb{R})_{0}$. The equivalence of the metric of Yang with the metric obtained via CS on $\mathcal{D}_{n}^{J}$ and then transported to $\mathcal{X}_{n}^{J}$ via partial Cayley transform is underlined in [17]. In particular, the metric (5.21c) appears in [110, p. 99] for the particular values $c_{1}=1, c_{2}=4$. See also $[109,111,112]$. 
Now we shall establish a metric invariant to the action given in Lemma 5.1 of $G_{1}^{J}(\mathbb{R})$ on the extended Siegel-Jacobi upper half-plane $\tilde{\mathcal{X}}_{1}^{J}$. Because the manifold $\tilde{\mathcal{X}}_{1}^{J}$ is 5-dimensional, we want to see if the extended Siegel-Jacobi upper half-plane is a Sasaki manifold, as in the case of $\mathrm{SL}(2, \mathbb{R})$ in Proposition 4.1. If we take as contact form $\eta=\lambda_{6}$, then $\mathrm{d} \eta=-2 \sqrt{\delta} \mathrm{d} p \wedge \mathrm{d} q$, and $\eta(\wedge \eta)^{2}=0$. If we try to determine a contact distribution $\mathcal{D}=\operatorname{Ann}(\eta)$, we get $\mathcal{D}=$ $\left\langle\frac{\partial}{\partial p}-q \frac{\partial}{\partial \theta}, \frac{\partial}{\partial q}+p \frac{\partial}{\partial \theta}\right\rangle$. From (D.4), we find $\Phi_{\lambda}^{\kappa}=0$, and $\Phi_{p}^{\kappa}=q \Phi_{\kappa}^{\lambda}, \Phi_{q}^{\lambda}=-p \Phi_{\kappa}^{\lambda}$, where $\lambda=x, y, p, q, \kappa$. So $\Phi$ has $\operatorname{Rank}(\Phi)<4$. In conclusion, $(\Phi, \xi, \eta)$ chosen as above can not be an almost contact structure for the extended Siegel-Jacobi upper half-plane $\tilde{\mathcal{X}}_{1}^{J}$.

We obtain

Proposition 5.6. The metric on the extended Siegel-Jacobi upper half-plane $\tilde{\mathcal{X}}_{1}^{J}$, in the partial $S$-coordinates $(x, y, p, q, \kappa)$ :

$$
\begin{aligned}
\mathrm{d} s_{\tilde{\mathcal{X}}_{1}^{J}}^{2}= & \mathrm{d} s_{\mathcal{X}_{1}^{J}}^{2}(x, y, p, q)+\lambda_{6}^{2}(p, q, \kappa) \\
= & \frac{\alpha}{y^{2}}\left(\mathrm{~d} x^{2}+\mathrm{d} y^{2}\right)+\left[\frac{\gamma}{y}\left(x^{2}+y^{2}\right)+\delta q^{2}\right] \mathrm{d} p^{2}+\left(\frac{\gamma}{y}+\delta p^{2}\right) \mathrm{d} q^{2}+\delta \mathrm{d} \kappa^{2} \\
& +2\left(\gamma \frac{x}{y}-\delta p q\right) \mathrm{d} p \mathrm{~d} q+2 \delta(q \mathrm{~d} p \mathrm{~d} \kappa-p \mathrm{~d} q \mathrm{~d} \kappa)
\end{aligned}
$$

is left-invariant with respect to the action given in Lemma 5.1 of the Jacobi group $G_{1}^{J}(\mathbb{R})$.

The matrix attached to metric (5.25) is

$$
\begin{aligned}
& g_{\tilde{\mathcal{X}}_{1}^{J}}=\left(\begin{array}{ccccc}
g_{x x} & 0 & 0 & 0 & 0 \\
0 & g_{y y} & 0 & 0 & 0 \\
0 & 0 & g_{p p}^{\prime} & g_{p q}^{\prime} & g_{p \kappa}^{\prime} \\
0 & 0 & g_{q p}^{\prime} & g_{q q}^{\prime} & g_{q \kappa}^{\prime} \\
0 & 0 & g_{\kappa p}^{\prime} & g_{\kappa q}^{\prime} & g_{\kappa \kappa}^{\prime}
\end{array}\right), \\
& g_{p q}^{\prime}=g_{p q}-\delta p q, \quad g_{p \kappa}^{\prime}=\delta q, \quad g_{q \kappa}^{\prime}=-\delta p, \quad g_{p p}^{\prime}=g_{p p}+\delta q^{2}, \\
& g_{q q}^{\prime}=g_{q q}+\delta p^{2}, \quad g_{\kappa \kappa}^{\prime}=\delta,
\end{aligned}
$$

while $g_{x x}, g_{y y}, g_{p p}, g_{q q}, g_{p q}$ are given in the metric matrix (5.22) associated with the balanced metric $(5.21 \mathrm{~b})$ on $\mathcal{X}_{1}^{J}$.

The metric (5.26) is orthonormal with respect to the invariant vector fields $L_{0}^{i}, i=1,2$, $L^{i}, i=4,5,6$. The fundamental vector fields with respect to the action (5.8) in the variables $(x, y, p, q, \kappa)$ are given by (5.14).

The extended Siegel-Jacobi upper half-plane $\tilde{\mathcal{X}}_{1}^{J}$ does not admit an almost contact structure $(\Phi, \xi, \eta)$ with a contact form $\eta=\lambda_{6}$ and Reeb vector $\xi=\operatorname{Ker}(\eta)$.

With $(5.15 \mathrm{c}),(5.15 \mathrm{e}),(5.15 \mathrm{f})$, we find the invariant metric on the Jacobi group $G_{1}^{J}(\mathbb{R})$ :

Theorem 5.7. The composition law for the real Jacobi group $G_{1}^{J}(\mathbb{R})$ in the $S$-coordinates $(x, y, \theta, p, q, \kappa)$ is given by (4.7) for $(x, y, \theta)$, (5.6) for $(p, q)$ and (5.8) for the coordinate $\kappa$, replacing in the matrix $M \in \mathrm{SL}(2, \mathbb{R})$ the values of $a, b, c$, $d$ as function of $(x, y, \theta)$ given by (4.4).

The left-invariant metric on the real Jacobi group $G_{1}^{J}(\mathbb{R})$ in the $S$-coordinates $(x, y, \theta, p, q, \kappa)$ is

$$
\begin{aligned}
\mathrm{d} s_{G_{1}^{J}(\mathbb{R})}^{2}= & \sum_{i=1}^{6} \lambda_{i}^{2}=\alpha \frac{\mathrm{d} x^{2}+\mathrm{d} y^{2}}{y^{2}}+\beta\left(\frac{\mathrm{d} x}{y}+2 \mathrm{~d} \theta\right)^{2} \\
& +\frac{\gamma}{y}\left[\mathrm{~d} q^{2}+\left(x^{2}+y^{2}\right) \mathrm{d} p^{2}+2 x \mathrm{~d} p \mathrm{~d} q\right]+\delta(\mathrm{d} \kappa-p \mathrm{~d} q+q \mathrm{~d} p)^{2},
\end{aligned}
$$


where $\lambda_{1}, \ldots, \lambda_{3}$ are defined by (4.10), while $\lambda_{4}, \ldots, \lambda_{6}$ are defined by (5.17), (5.15c)-(5.15f). The matrix attached to the metric (5.27) in the variables $(x, y, \theta, p, q, \kappa)$ reads

$$
g_{G_{1}^{J}}=\left(\begin{array}{cccccc}
g_{x x} & 0 & g_{x \theta} & 0 & 0 & 0 \\
0 & g_{y y} & 0 & 0 & 0 & 0 \\
g_{\theta x} & 0 & g_{\theta \theta} & 0 & 0 & 0 \\
0 & 0 & 0 & g_{p p}^{\prime} & g_{p q}^{\prime} & g_{p \kappa}^{\prime} \\
0 & 0 & 0 & g_{q p}^{\prime} & g_{q q}^{\prime} & g_{q \kappa}^{\prime} \\
0 & 0 & 0 & g_{\kappa p}^{\prime} & g_{\kappa q}^{\prime} & g_{\kappa \kappa}^{\prime}
\end{array}\right),
$$

where $g_{x x}, g_{y y}, g_{x \theta}, g_{\theta \theta}$ are those attached to $\mathrm{SL}(2, \mathbb{R})$ given by $(4.21), g_{p p}^{\prime}, g_{p q}^{\prime}, g_{q q}^{\prime}, g_{p \kappa}^{\prime}, g_{q \kappa}^{\prime}$, $g_{\kappa \kappa}^{\prime}$ are given by (5.26).

We have

$$
\left\langle\lambda_{i} \mid L^{j}\right\rangle=\delta_{i j}, \quad i, j=1, \ldots, 6,
$$

where $L^{1}, \ldots, L^{3}$ are defined by (4.11), while $L^{4}, \ldots, L^{6}$ are defined by (5.18), (5.16b)-(5.16f). The vector fields $L^{i}, i=1, \ldots, 6$ verify the commutations relations (5.19) and are orthonormal with respect to the metric (5.27).

Depending of the values of the parametres $\alpha, \beta, \gamma, \delta$, we have invariant metric on the following manifolds:

1) the Siegel upper half-plane $\mathcal{X}_{1}$ if $\beta, \gamma, \delta=0$, see Proposition 4.1,

2) the group $\mathrm{SL}(2, \mathbb{R})$ if $\gamma, \delta=0, \beta \neq 0$, see Proposition 4.1,

3) the Siegel-Jacobi half-plane $\mathcal{X}_{1}^{J}$ if $\beta, \delta=0$, see Proposition 5.4,

4) the extended Siegel-Jacobi half-plane $\tilde{\mathcal{X}}_{1}^{J}$ if $\beta=0$, see Proposition 5.6,

5) the Jacobi group $G_{1}^{J}$ if $\alpha \beta \gamma \delta \neq 0$.

We show some consequences of Theorem 5.7. We investigate if the homogeneous manifold $\mathcal{X}_{1}^{J}$ is a naturally reductive manifold or not. The fact that $\mathcal{X}_{1}^{J}$ is not a naturally reductive 4dimensional manifold is well known, see Theorem A.12, but in Proposition 5.8 below we present a direct proof.

Proposition 5.8. The Siegel-Jacobi upper half-plane realized as homogenous Riemannian manifold $\left(\mathcal{X}_{1}^{J}=\frac{G_{1}^{J}(\mathbb{R})}{\mathrm{SO}(2) \times \mathbb{R}}, g_{\mathcal{X}_{1}^{J}}\right)$ is a reductive, non-symmetric manifold, not naturally reductive with respect to the balanced metric $(5.21 \mathrm{~b})$.

The Siegel-Jacobi upper half-plane $\mathcal{X}_{1}^{J}$ is not a g.o. manifold with respect to the balanced metric.

When expressed in the variables that appear in the FC-transform given in Proposition 2.1, $\mathcal{X}_{1}^{J}$ is a naturally reductive space with the metric $g_{\mathcal{X}_{1}} \times g_{\mathbb{R}^{2}}$, where $g_{\mathcal{X}_{1}}$ is given by $(4.22)$ and $g_{\mathbb{R}^{2}}$ is the Euclidean metric (A.14).

If

$$
\mathfrak{g}_{1}^{J} \ni X=a L^{1}+b L^{2}+c L^{3}+d L^{4}+e L^{5}+f L^{6},
$$

then a geodesic vector of the homogeneous manifold $\mathcal{X}_{1}^{J}$ has one of the following expressions given in Table 1.

Proof. From the commutation relations (C.11), (3.2), (5.1), it is seen that for $\mathfrak{g}_{1}^{J}(\mathbb{R})$, we have

$$
\mathfrak{m}=\langle F, G, P, Q\rangle, \quad \mathfrak{h}=\langle H, R\rangle,
$$

because $[\mathfrak{m}, \mathfrak{h}] \subset \mathfrak{m}$, i.e., $\mathcal{X}_{1}^{J}$ is a reductive space, cf. Definition A.5. 
Table 1. Components of the geodesic vector (5.28).

\begin{tabular}{||c|c|c|c|c|c|c||}
\hline Nr. cr. & $\mathrm{a}$ & $\mathrm{b}$ & $\mathrm{c}$ & $\mathrm{d}$ & $\mathrm{e}$ & $\mathrm{f}$ \\
\hline 1 & 0 & 0 & $c$ & 0 & 0 & $f$ \\
\hline 2 & $a$ & $b$ & 0 & 0 & 0 & $f$ \\
\hline 3 & $r c$ & 0 & $c$ & $\pm r c$ & 0 & $f$ \\
\hline 4 & $a$ & 0 & $-a$ & 0 & $\epsilon \sqrt{r} a$ & $f$ \\
\hline 5 & $\epsilon_{1} \epsilon_{2} \frac{1-r}{\sqrt{r}} e$ & $\epsilon_{1} e$ & $-\frac{\epsilon_{1} \epsilon_{2}}{\sqrt{r}} e$ & $\epsilon_{2} \sqrt{r} e$ & $e$ & $f$ \\
\hline
\end{tabular}

Here $r=\sqrt{\frac{\alpha}{\beta}}, \epsilon_{1}^{2}=\epsilon_{2}^{2}=\epsilon^{2}=1$.

But $[\mathfrak{m}, \mathfrak{m}] \nsubseteq \mathfrak{h}$, and $\mathcal{X}_{1}^{J}$ is not a symmetric manifold.

We verify (A.10) written as

$$
g\left(\left[X_{1}, X_{3}\right]_{\mathfrak{m}}, X_{2}\right)+g\left(X_{1},\left[X_{3}, X_{2}\right]_{\mathfrak{m}}\right)=0, \quad \forall X_{1}, X_{2}, X_{3} \in \mathfrak{m} .
$$

Instead of (5.29) we take

$$
\mathfrak{m}=\left\langle L^{1}, L^{2}, L^{4}, L^{5}\right\rangle, \quad \mathfrak{h}=\left\langle L^{3}, L^{6}\right\rangle,
$$

where $L^{1}, \ldots, L^{3}\left(L^{4}, \ldots, L^{6}\right)$ are defined in (4.11), (respectively (5.18)).

We take

$$
X_{i}=a_{i} L^{1}+b_{i} L^{2}+c_{i} L^{4}+d_{i} L^{5}, \quad i=1,2,3,
$$

and, with the commutation relations (5.19), we find

$$
\left[X_{1}, X_{3}\right]=\frac{1}{2 \sqrt{\alpha}}\left[\left(d_{1} a_{3}-a_{1} d_{3}+c_{1} b_{3}-b_{1} c_{3}\right) L^{4}+\left(c_{1} a_{3}-a_{1} c_{3}+b_{1} d_{3}-d_{1} b_{3}\right) L^{5}\right] .
$$

Taking into account that the vector fields $L^{1}, \ldots, L^{6}$ are orthonormal with respect to the metric (5.27) on $G_{1}^{J}$ as in Theorem 5.7, the condition (5.30) of the geodesic Lemma A.19 reads

$$
c_{3}\left(a_{2} d_{1}-a_{1} d_{2}+b_{2} c_{1}-b_{1} c_{2}\right)+d_{3}\left(b_{1} d_{2}-b_{2} d_{1}+a_{2} c_{1}-a_{1} c_{2}\right)=0 .
$$

The condition (5.31) implies that the system of algebraic equations

$$
\begin{aligned}
& a_{1} d_{2}+b_{1} c_{2}=c_{1} b_{2}+d_{1} a_{2}, \\
& a_{1} c_{2}-b_{1} d_{2}=c_{1} a_{2}-d_{1} b_{2},
\end{aligned}
$$

must have a solution for any $a_{i}, b_{i}, c_{i}, d_{i}, i=1,2,3$, which is not possible, and $\mathcal{X}_{1}^{J}$ is not naturally reductive with respect to the balanced metric.

Due to Theorem A.20, the four-dimensional manifold $\mathcal{X}_{1}^{J}$ is not a g.o. manifold.

We also recall that in [17, Propositions 3 and 4] it was proved that under the so called FCtransform, the manifold $\mathcal{X}_{n}^{J}$ is symplectomorph with $\mathcal{X}^{n} \times \mathbb{C}^{n}$. The particular case of the Jacobi group of degree 1 was reproduced in Proposition 2.1 and, in particular, $\mathcal{X}_{1}^{J}$ is equivalent with the symmetric space $\mathcal{X}_{1} \times \mathbb{C}$, which is naturally reductive, as in Theorem A.12.

To find the geodesic vectors on the Siegel-Jacobi upper half-plane $\mathcal{X}_{1}^{J}$, we look for the solution (5.28) that verifies the condition (A.22) of the geodesic lemma expressed in Proposition A.19. Taking

$$
\mathfrak{m} \ni Y=a_{1} L^{1}+b_{1} L^{2}+d_{1} L^{4}+e_{1} L^{5},
$$


the condition (A.22)

$$
\begin{aligned}
a_{1}\left(\frac{b c}{\sqrt{\beta}}+\frac{e d}{\sqrt{\alpha}}\right)+\frac{b_{1}}{2}\left[-\frac{a c}{\sqrt{\beta}}+\frac{1}{\sqrt{\alpha}}\left(d^{2}-e^{2}\right)\right] \\
\quad-\frac{d_{1}}{2 \sqrt{\alpha}}(b d+e c+a e)+\frac{e_{1}}{2}\left[\frac{c d}{\sqrt{\beta}}+\frac{1}{\sqrt{\alpha}}(b e-a d)\right]=0
\end{aligned}
$$

must be satisfied for every values of $a_{1}, b_{1}, d_{1}, e_{1}$, i.e., the coefficients of the geodesic vector (5.28) are solutions of the system of algebraic equations

$$
\begin{aligned}
& r b c+d e=0, \\
& -r a c+d^{2}-e^{2}=0, \\
& b d+e(a+c)=0, \\
& r c d+b e-a d=0 .
\end{aligned}
$$

The solutions of the system (5.32) are written in Table 1.

\section{A Naturally reductive spaces}

\section{A.1 Fundamental vector fields}

A homogeneous space is a manifold $M$ with a transitive action of a Lie group $G$. Equivalently, it is a manifold of the form $G / H$, where $G$ is a Lie group and $H$ is a closed subgroup of $G$, cf., e.g., [6, p. 67].

Let $(M, g),\left(N, g^{\prime}\right)$ be Riemannian manifolds. An isometry is a diffeomorphism $f: M \rightarrow N$ that preserves the metric, i.e., $g_{p}(u, v)=g_{f(p)}^{\prime}\left(\mathrm{d} f_{p}(u), \mathrm{d} f_{p}(v)\right), \forall p \in M, \forall u, v \in T M_{p}$. If $(M, g)$ is a Riemannian manifold, the set $I(M, g)$ (or $I(M)$ ) of all isometries $M \rightarrow M$ forms a group called the isometry group of $M$.

A Riemannian homogenous space is a Riemannian manifold $(M, g)$ on which the isometry group $I(M)$ acts transitively. A Riemannian manifold $(M, g)$ is a $G$-homogenous (or homogenous under a Lie group $G)$ if $G$ is a closed subgroup of $I(M, g)$ which acts transitively on $M$, cf. [40, p. 178].

Let $G$ be a Lie group of transformations acting on the manifold $M$, cf. [63, Chapter II, Section 3, p. 121]. In [63, p. 122] it is introduced the notion of vector field on $M$ induced by the one parameter subgroup $\exp t X, t \in \mathbb{R}, X \in \mathfrak{g}$, denoted $X^{+}$, where $\mathfrak{g}$ is the Lie algebra of $G$. In [70, Section 5, p. 51], in the context of principal fibre bundle $P(M, G)$ over $M$ with structure group the Lie group $G$, it is introduced the same notion under the name fundamental vector field associate to $X \in \mathfrak{g}$, denoted $X^{*}$, see also [70, Proposition 4.1, p. 42].

Let $M=G / H$ be a homogeneous $n$-dimensional manifold and let us suppose that $G$ acts transitively on the left on $M, G \times M \rightarrow M: g \cdot x=y$, where $y=\left(y_{1}, \ldots, y_{n}\right)^{t}$. Then $g(t) \cdot x=y(t)$, where $g(t)=\exp (t X), t \in \mathbb{R}$, generates a curve in $M$ with $y(0)=x$ and $\dot{y}(0)=X$. The fundamental vector field attached to $X \in \mathfrak{g}$ at $x \in M$ is defined as

$$
X_{x}^{*}:=\left.\frac{\mathrm{d}}{\mathrm{d} t} y(t)\right|_{t=0}=\left.\frac{\mathrm{d}}{\mathrm{d} t}(\exp (t X) \cdot x)\right|_{t=0} .
$$

We write the fundamental vector field attached to $X \in \mathfrak{g}$ as

$$
X_{x}^{*}=\sum_{i=1}^{n}\left(X_{i}^{*}\right)_{x} \frac{\partial}{\partial z_{i}}, \quad\left(X_{i}^{*}\right)_{x}=\left.\frac{\mathrm{d} y_{i}(t)}{\mathrm{d} t}\right|_{t=0} .
$$

Now, because $\left[X^{*}, Y^{*}\right]=-[X, Y]^{*}$, see, e.g., in $[63$, Theorem 3.4, p. 122], it is observed 
Lemma A.1. If the generators $X_{1}, \ldots, X_{n}$ of a Lie algebra $\mathfrak{g}$ verify the commutations relations

$$
\left[X_{i}, X_{j}\right]=c_{i j}^{k} X_{k}
$$

then the associated fundamental vector fields verify the commutation relations

$$
\left[X_{i}^{*}, X_{j}^{*}\right]=-c_{i j}^{k} X_{k}^{*}
$$

Note that if the action of $G$ on $M$ is on the right as in [70, p. 51], then

$$
\left[X_{i}^{*}, X_{j}^{*}\right]=c_{i j}^{k} X_{k}^{*}
$$

\section{A.2 Killing vectors}

A vector field $X \in \mathfrak{D}^{1}(M)$ on a Riemannian manifold $(M, g)$ is called an infinitesimal isometry or a Killing vector field if the local 1-parameter group of local transformations by $X$ in a neighbourhood of each point of $M$ consists of local isometries, see also in [70, Proposition 3.2, p. 237], i.e.,

$$
L_{X} g=0, \quad X \in \mathfrak{D}^{1}(M),
$$

where $L_{X}$ is the Lie derivative on $M$.

We recall below in Lemma A.2 the Killing equations (A.5), see, e.g., [113, Theorem 1.3, p. 5] or [104, equation $\left(40^{\prime}\right)$, p. 247]. We use the tensor notation as in [104, 113].

Let us consider a $n$-dimensional Riemannian manifold $(M, g)$ and a vector field with the contravariant components $X^{i}, i=1, \ldots, n$ :

$$
X=\sum_{i=1}^{n} X^{i} \frac{\partial}{\partial x^{i}}
$$

If $\nabla$ denotes the covariant derivative, we have the standard formulas

$$
\begin{aligned}
& \nabla_{\mu} g_{\lambda \chi}:=\partial_{\mu} g_{\lambda \chi}-\Gamma_{\mu \lambda}^{\rho} g_{\rho \chi}-\Gamma_{\mu \chi}^{\rho} g_{\lambda \rho}, \\
& \nabla_{\mu} X^{\chi}:=\partial_{\mu} X^{\chi}+\Gamma_{\mu \lambda}^{\chi} X^{\lambda}, \\
& \nabla_{\mu} X_{\chi}:=\partial_{\mu} X_{\chi}-\Gamma_{\mu \chi}^{\lambda} X_{\lambda}, \\
& X_{\mu}:=g_{\mu \lambda} X^{\lambda}, \\
& L_{X} g_{\lambda \chi}:=X^{\mu} \partial_{\mu} g_{\lambda \chi}+g_{\rho \chi} \partial_{\lambda} X^{\rho}+g_{\lambda \rho} \partial_{\chi} X^{\rho} .
\end{aligned}
$$

Lemma A.2. Let $(M, g)$ be a n-dimensional Riemannian manifold with a Riemannian (metric) connection. The field $X$ is a Killing vector field if and only if its covariant components $X_{\mu}$, $\mu=1, \ldots, n$ verify the Killing equations

$$
\nabla_{\lambda} X_{\chi}+\nabla_{\chi} X_{\lambda}:=\frac{\partial X_{\chi}}{\partial x^{\lambda}}+\frac{\partial X_{\lambda}}{\partial x^{\chi}}-2 \Gamma_{\lambda \chi}^{\rho} X_{\rho}=0, \quad \lambda, \chi=1, \ldots, n .
$$

Remark A.3. If the coordinate $x^{\alpha}$ is not present in the expression of metric tensor $g_{\lambda \chi}, \lambda, \chi=$ $1, \ldots, n$, then $\frac{\partial}{\partial x^{\alpha}}$ is a Killing vector field for the metric $g_{\lambda \chi}$.

With (A.4e), the condition (A.2) of a vector field (A.3) to be a Killing vector field is that its contravariant components to verify the equations

$$
X^{\mu} \partial_{\mu} g_{\lambda \chi}+g_{\mu \chi} \partial_{\lambda} X^{\mu}+g_{\lambda \mu} \partial_{\chi} X^{\mu}=0, \quad \lambda, \chi, \mu=1, \ldots, \operatorname{dim} M=n
$$


The system (A.6) of $n(n+1) / 2$ equations of a Killing vector field $X^{1}(x), \ldots, X^{n}(x)$ is overdetermined, and no-nonvanishing solution is guaranteed, in general. The set $\iota(M)$ of all Killing vector fields on $n$-dimensional manifold $M$ forms a Lie algebra of dimension not exceeding $\frac{n(n+1)}{2}$ and $\operatorname{dim}(\iota(M))=\frac{n(n+1)}{2}$ is obtained only for spaces of constant curvature, see [70, Theorem 3.3, p. 238]. For example, maximal solution is obtained for the (pseudo)-Euclidean spaces $E^{r, n-r}$, for the sphere $S^{n}$ or the real projective space $R P^{n}=S^{n} /( \pm I)$, see, e.g., [70, Theorem 1, p. 308], [104, p. 251] and [57, Section 4.6.6, p. 83]. We have $\iota\left(E^{r, s}\right)=\mathfrak{s o}(r, s) \ltimes \mathbb{R}^{r+s}$. The Euclidean group $E^{n}$ of $\mathbb{R}^{n}$ has dimension $n(n+1) / 2$, where $n$ degrees of freedom correspond to translations, the other $n(n-1) / 2$ correspond to rotations, see also Proposition A.13 and Remark C.4 below.

The following remark is very important for the determination of Killing vector fields on Riemannian homogeneous manifolds, see, e.g., see [35, p. 4] or [72, Proposition 2.2, p. 139]:

Remark A.4. If $(M, g)$ is a Riemannian homogeneous space $M=G / H$ endowed with a $G$ invariant Riemannian metric $g$, then each $X \in \mathfrak{g}$ generates a one-parameter subgroup of the group $I(M)$ of isometries (motions) of $M$ via $p \rightarrow(\exp t X) \cdot p$. Hence the fundamental vector field $X^{*}$ on a Riemannian homogeneous manifold is a Killing vector. For Riemannian homogeneous spaces $M=G / H$, $\operatorname{dim}(I(M))=\operatorname{dim}(G)$.

\section{A.3 Reductive homogeneous spaces}

The following notions are standard, see [63, pp. 121, 123 and 125] or [70, p. 155] and [71, p. 187]; see also $[3,89,90]$.

The set of elements $G_{x}$ of a given group $G$, acting on a set $M$ as group of transformations that leaves the point $x$ fixed, is called isotropy group, also called stationary group or stabilizer. If $G$ is a Lie group and $H$ is a closed subgroup, then the coset space $G / H$, in particular, $H=G_{x}$ is taken with the analytic structure given in [63, Theorem 4.2, p. 123]. For $x \in G$, the diffeomorphism of $G / H$ into itself is $\tau(x): y H \rightarrow x y H$. The natural representation of the isotropy group of a differentiable transformation group in the tangent space to the underling manifold is called isotropy representation. If $G$ is the group of differentiable transformations on the manifold $M$ and $G_{x}$ is the corresponding isotropy subgroup at the point $x \in M$, then the isotropy representation $\mathrm{Is}_{x}: G_{x} \rightarrow \mathrm{GL}\left(T_{x} M\right)$ associates to each $h \in G_{x}$ the differential $\mathrm{Is}_{x}(h):=$ $(\mathrm{d} \tau(h))_{\lambda(H)}$ of the transformation $h$ at $x$, where $\lambda: G \rightarrow G / H$ is the canonical projection. The image of the isotropy representation, $\operatorname{Is}_{x}\left(G_{x}\right)$, is called the linear isotropy group at $x$.

If $G$ is a Lie group with a countable base acting transitively and smoothly on $M$, then the tangent space $T_{x} M$ can be naturally identified with the space $\mathfrak{g} / \mathfrak{g}_{x}$, where $\mathfrak{g} \supset \mathfrak{g}_{x}$ are respectively the Lie algebras of the groups $G \supset G_{x}$. The isotropy representation $\mathrm{Is}_{x}$ is now identified with the representation $G_{x} \rightarrow \mathrm{GL}\left(\mathfrak{g} / \mathfrak{g}_{x}\right)$, induced by the restriction of the adjoint representation $\operatorname{Ad}_{G}$ of $G$ to $G_{x}$. See details below in Lemma A.6.

Definition A.5 (cf. Nomizu [88]). A homogeneous space $M=G / H$ is reductive if the Lie algebra $\mathfrak{g}$ of $G$ may be decomposed into a vector space direct sum of the Lie algebra $\mathfrak{h}$ of $H$ and an $\operatorname{Ad}(H)$-invariant subspace $\mathfrak{m}$, that is

$$
\begin{aligned}
& \mathfrak{g}=\mathfrak{h}+\mathfrak{m}, \quad \mathfrak{h} \cap \mathfrak{m}=\varnothing, \\
& \operatorname{Ad}(H) \mathfrak{m} \subset \mathfrak{m} .
\end{aligned}
$$

Condition (A.7b) implies

$$
[\mathfrak{h}, \mathfrak{m}] \subset \mathfrak{m}
$$

and, conversely, if $H$ is connected, then (A.7c) implies (A.7b). Note that $H$ is always connected if $M$ is simply connected. The decomposition (A.7a) verifying (A.7b) is called a $H$-stable decomposition. 
Lemma A.6. If a homogeneous space $M$ is reductive, then $T_{x} M$ can be identified with $\mathfrak{m}$, while $\mathrm{Is}_{x}$ can be identified with the representation $\left.h \mapsto\left(\operatorname{Ad}_{G} h\right)\right|_{\mathfrak{m}}$. In this case, the isotropy representation is faithful if $G$ acts effectively.

So let us denote by $x(s)$ the 1-parameter subgroup of $G$ generated by $X \in \mathfrak{m}$ and let $x^{*}(s)=$ $\lambda(x(s))$ be the image of $x(s)$ by the projection $\lambda$ of $G$ onto $G / H$ :

$$
x^{*}(s)=x(s) . o=\left(\exp s X^{*}\right) . o .
$$

Identifying $X^{*}$ with $X \in \mathfrak{m}$, we can write down

$$
x^{*}(s)=\exp (s X) \cdot o .
$$

The invariant tensor fields on a homogeneous space $M$ are in one-to-one correspondence with the tensor fields on $T_{x} M$ that are invariant with respect to the isotropy representation. In particular, $M$ has an invariant Riemannian metric if and only if $T_{x} M$ has a Euclidean metric that is invariant under the linear isotropy group.

In accord with [71, Proposition 3.1 and Corollary 3.2, p. 200] and [6, p. 78]:

Proposition A.7. Let $M=G / H$ be a homogenous space where $G$ is a Lie group acting effectively on $M$, which is reductive.

The one-to-one correspondence between $G$-invariant indefinite Riemannian metrics $g$ on $M=$ $G / H$ and $\operatorname{Ad}(H)$-invariant non-degenerate symmetric bilinear forms $B$ on $\mathfrak{m}$

$$
B(X, Y)=B\left(\operatorname{Ad}^{G / H}(h) X, \operatorname{Ad}^{G / H}(h) Y\right), \quad \forall X, Y \in \mathfrak{m}, h \in H,
$$

is given by

$$
B(X, Y)=g\left(X^{*}, Y^{*}\right)_{o}, \quad \text { for } \quad X, Y \in \mathfrak{m},
$$

Explicitly, the $\operatorname{Ad}(H)$ invariance of the symmetric non-degenerate form $B$ in (A.8) means, see, e.g., [71, p. 201]

$$
B(X,[Z, Y])+B([Z, X], Y)=0, \quad X, Y \in \mathfrak{m}, \quad Z \in \mathfrak{h} .
$$

Usually it is asked that the group of isometries $G$ acts effectively on $M$, cf. [51].

The canonical connection, see [71, p. 192], or canonical affine connection of second type, see [88], on the reductive space $M=G / H$ verifying (A.7a), (A.7b), is the unique $G$-invariant affine connection on $M$ such that for any vector field $X \in \mathfrak{m}$ and any frame $u$ at the point $o$, the curve $(\exp t X) u$ in the principal fibration of frames over $M$ is horizontal. The canonical connection is complete and the set of its geodesics through $o$ coincides with the set of curves of the type $(\exp t X) o$, where $X \in \mathfrak{m}$, see also [71, Proposition 2.4 and Corollary 2.5, p. 192]. In a reductive space there is a unique $G$-invariant affine connection with zero torsion having the same geodesics as the canonical connection, cf. [71, Theorem 2.1, p. 197]. This connection is called in [71] natural torsion-free connection on $M=G / H$ relative to the decomposition (A.7a), or canonical affine connection of the first kind in [88].

\section{A.4 Naturally reductive spaces}

For the next definition and (A.10) below, see in [88, Chapter II, Section 13, metric connections], [71, p. 202] and [51], 
Definition A.8. A homogeneous Riemannian or pseudo-Riemannian space $M=G / H$ is naturally reductive if it is reductive, i.e., it verifies (A.7a), (A.7b), and

$$
B\left(X,[Z, Y]_{\mathfrak{m}}\right)+B\left([Z, X]_{\mathfrak{m}}, Y\right)=0, \quad X, Y, Z \in \mathfrak{m},
$$

where $B$ is the non-degenerate symmetric bilinear form on $\mathfrak{m}$ induced by the Riemannian (pseudo-Riemannian) structure on $M$ under the natural identification of the spaces $\mathfrak{m}$ and $M_{o}$, as in (A.8).

If $M=G / H$ is a naturally reductive Riemannian or pseudo-Riemannian space verifying (A.7a), (A.7b), and (A.10), then the natural torsion-free connection coincides with the corresponding Riemannian or pseudo-Riemannian connection on $M$ [3].

Based on [4, Theorem 5.4], [71, Chapter X, Section 3], [100, Theorem 6.2, p. 58] and [35, Proposition 1, p. 5], it is formulated the following

Proposition A.9. Let $(M, g)$ be a homogeneous Riemannian manifold. Then $(M, g)$ is a naturally reductive Riemannian homogenous space if and only if there exists a connected Lie subgroup $G$ of $I(M)$ acting transitively and effectively on $M$ and a reductive decomposition (A.7a), such that one of the following equivalent statements hold:

(i) (A.10), or

$$
g\left([X, Z]_{\mathfrak{m}}, Y\right)+g\left(X,[Z, Y]_{\mathfrak{m}}\right)=0 \quad \forall X, Y, Z \in \mathfrak{m},
$$

is verified;

(ii) the Levi-Civita connection of $(M, g)$ and the natural torsion-free connection with respect to the decomposition (A.7a) are the same;

(iii) (*) is true, i.e., every geodesic in $M$ is the orbit of a one-parameter subgroup of $I(M)$ generated by some $X \in \mathfrak{m}$.

It is not always easy to decide whether a given homogenous Riemannian space is naturally reductive [1]. The Riemannian manifold $M=G / H$ might be naturally reductive although for any reductive decomposition $\mathfrak{g}=\mathfrak{h}+\mathfrak{m}$ none of the statements in Proposition A.9 holds, because that might exist another appropriate subgroup $\tilde{G} \subset I(M)$ such that $M=\tilde{G} / \tilde{H}$ and with respect to such decomposition the conditions of Proposition A.9 are satisfied, see, e.g., [35, p. 5]. In accord with [35, Proposition 2, p. 5], a necessary and sufficient condition that a complete and simply connected manifold be naturally reductive is that there exists a homogeneous structure $T$ on $M$ with $T_{*} v=0$, for all tangent vectors $v$ of $M$.

Ambrose and Singer found the condition for a Riemannian manifold be locally homogeneous [4].

\section{A.5 Naturally reductive spaces of dimension $\leq 4$}

The connected homogeneous Riemannian $V_{n}$ naturally reductive spaces of dimension $n \leq 6$ are classified.

For two dimensional manifolds, because the homogeneous manifolds $V_{2}$ have constant curvature, they are locally symmetric spaces, see, e.g., in [100, Theorem 4.1, Section 4].

Theorem A.10. The only homogenous structure on $\mathbb{R}^{2}$ and $S^{2}$ is given by $T=0$, cf. [100, Corollary 4.2].

Let $(M, g)$ be a connected and simply connected surface. Then $(M, g)$ admits a homogenous structure $T \neq 0$ if and only if $(M, g)$ is isomorphic to the hyperbolic plane, cf. [35, Theorem 4.3].

Up to an isomorphism, $\mathcal{H}^{2}$ has only two homogenous structures, namely: 
1. $T=0$, corresponding to the symmetric case $\mathcal{H}^{2}=\mathrm{SO}_{0}(1,2) / \mathrm{U}(1)$, where $\mathrm{SO}_{0}(1,2)=$ $\mathrm{SL}(2, \mathbb{R}) / \pm I$ is the connected component of the identity of the Lorentz group, see also (C.14).

2. $T_{X} Y=g(X, Y) \xi-g(\xi, Y) X, \xi=\xi^{1} E_{1}+\xi^{2} E_{2}, E_{1}=\frac{1}{r} y^{1} \frac{\partial}{\partial y^{1}}, E_{2}=\frac{1}{r} y^{1} \frac{\partial}{\partial y^{2}}$,

$$
g=r^{2}\left(y^{1}\right)^{-2}\left(\left(\mathrm{~d} y^{1}\right)^{2}+\left(\mathrm{d} y^{2}\right)^{2}\right)
$$

$X, Y \in \mathfrak{D}^{1}(M), r>0$. This homogenous structure corresponds to the Lie algebra $\mathfrak{g}$ with the product $\left(y_{1}, y_{2}\right)\left(y_{1}^{\prime}, y_{2}^{\prime}\right)=\left(y_{1} y_{1}^{\prime}, y_{1} y_{2}^{\prime}+y_{2}\right)$, i.e., the semi-direct product of the multiplicative group $\mathbb{R}_{0}^{+}$and the additive group $\mathbb{R}$.

The case $n=3$ was considered by Kowalski [74]. The proof of Theorems A.11 and A.12 below is based on the Ambrose and Singer theorem in the formulation of [100, Section 2] and the classification of 3-dimensional unimodular Lie groups with left-invariant metrics of Milnor [83].

The following theorem is [100, Theorem 6.5, p. 63], [36, Theorem 2] or [1, Theorem 5.2]:

Theorem A.11. A three-dimensional complete, simply connected naturally reductive Riemannian manifold $(M, g)$ is either:

(a) a symmetric space realized by the real forms: $\mathbb{R}^{3}, S^{3}$ or the Poincaré half-space $\mathcal{H}^{3}$, and $S^{2} \times \mathbb{R}, \mathcal{H}^{2} \times \mathbb{R}$, or

(b) a non-symmetric space isometric to one of the following Lie groups with a suitable leftinvariant metric:

(b1) $\mathrm{SU}(2)$,

$(b 2) \widetilde{\mathrm{SL}}(2, \mathbb{R})$, the universal covering of $\mathrm{SL}(2, \mathbb{R})$, with a special left-invariant metric,

(b3) the 3-dimensional Heisenberg group $\mathrm{H}_{1}$, where the Heisenberg group has a left-invariant metric.

The Poincaré half-space $\mathcal{H}^{n}$ is the set $\left(x_{1}, \ldots, x_{n}\right) \in \mathbb{R}^{n}, x_{1}>0$, with the metric proportional with

$$
\mathrm{d} s^{2}:=x_{1}^{-2} \sum_{i=1}^{n}\left(\mathrm{~d} x_{i}\right)^{2} .
$$

For the left invariant Riemannian metrics which appear in Theorem A.11, see [74, Theorem 2] and [91]. For $H_{1}=\mathbb{R}^{3}[x, y, z]$ a left-invariant metric is

$$
\mathrm{d} s_{H_{3}}^{2}=\frac{1}{b}\left(\mathrm{~d} x^{2}+\mathrm{d} z^{2}+(\mathrm{d} y-x \mathrm{~d} z)^{2}\right), \quad b \in \mathbb{R}_{+} .
$$

Note that in [35, Theorem 1, p. 6] appear only the non-symmetric naturally reductive spaces of dimensions 3: $\mathrm{SU}(2) \cong S^{3}, \widetilde{\mathrm{SL}}(2, \mathbb{R})$ and $\mathrm{Nil}_{3}$. The metrics of these spaces are particular cases of the 7-families of BCV-spaces that appear in Theorem A.15, because the naturally reductive spaces are a particular class of homogenous spaces.

The case of four-dimensional manifolds was treated by Kowalski and Vanhecke, see [76, Theorem 1, p. 224] or [35, Theorem 2, p. 6]:

Theorem A.12. Let $(M, g)$ be a four-dimensional simply connected naturally reductive Riemannian manifold. Then $(M, g)$ is either symmetric or it is a Riemannian product of the naturally reductive spaces of dimension 3 of type $(b)$ appearing in Theorem A.11 times $\mathbb{R}$. In the last cases, $(M, g)$ is not locally symmetric. 


\section{A.6 $V_{2}$ and $V_{3}$ spaces with transitive group}

The determination of the groups $G_{3}$ of isometries with three parameters of a two-dimensional space $V_{2}$ with positive definite metric was done by Bianchi [41]. In Proposition A.13 below we follow Vranceanu, see [104, Chapter V, Section 14, p. 288]. The generators of $G_{3}$ in [104, equation (90)] considered by Vranceanu, in our notation (C.37), verifies the commutation relations

$$
[X, Y]=-\epsilon Z, \quad[Y, Z]=-k X, \quad[Z, X]=-Y, \quad \epsilon= \pm 1 .
$$

Below we also write down $V_{2}$ as a homogenous manifolds.

$\mathrm{E}(2)$ is the group of rigid motions of the Euclidean 2-space, denoted $M(2)$ in [102, p. 195], see also [102, Section 8.5].

\section{Proposition A.13.}

1. If $k=0, \epsilon=1$, then the invariant metric of $V_{2}$ is given by (A.14),

$$
\mathrm{d} s^{2}=\mathrm{d} x^{2}+\mathrm{d} y^{2}
$$

and $V_{2}$ is the Euclidean space $E^{2}=E(2) / O(2)$. The Euclidean group is $E(2)=\mathbb{R}^{2} \rtimes O(2)$.

2. If $k=0, \epsilon=-1$, then the invariant metric of $V_{2}$ is

$$
\mathrm{d} s^{2}=\mathrm{d} x^{2}-\mathrm{d} y^{2}
$$

on the pseudo-euclidean space $V_{2}=E^{1,1}=E(1,1) / O(1,1), E(1,1)=\mathbb{R}^{2} \rtimes O(1,1)$.

3. A space $V_{2}$ with group $G_{3}$ always admits a simply transitively subgroup, except when the generators (A.13) of the structure group for $\epsilon=1$ and $k>0$, when the stereographic projection of the sphere from the south pole $(0,0,-R)$ to plane tangent in the north pole $(0,0, R)$ has the expression (C.8), where $m=\frac{k}{4}$, and the generators (C.36) are

$$
X=\sqrt{k}\left(z \frac{\partial}{\partial x}-x \frac{\partial}{\partial z}\right), \quad Y=\sqrt{k}\left(-y \frac{\partial}{\partial z}+z \frac{\partial}{\partial y}\right), \quad Z=y \frac{\partial}{\partial x}-x \frac{\partial}{\partial y},
$$

i.e., rotations around the axes $x, y, z$. We have $V_{2}=S^{2} \cong \mathbb{C P}^{1} \cong \mathrm{SU}(2) / \mathrm{U}(1)$. The metric of a space $V_{2}$ with simply transitive abelian group may be written as

$$
\mathrm{d} s^{2}=\mathrm{e}^{2 \lambda v} \mathrm{~d} u^{2}+\epsilon \mathrm{d} v^{2}, \quad k=-\lambda^{2} .
$$

If $\epsilon=1$ and $k>0$, then (A.16) can be written down as

$$
\mathrm{d} s^{2}=\mathrm{d} u^{2}+\mathrm{d} v^{2}+\frac{k(u \mathrm{~d} v+v \mathrm{~d} u)^{2}}{1-k\left(u^{2}+v^{2}\right)}, \quad k=\frac{1}{R^{2}} .
$$

4. If $\epsilon=1$ and $k<0$, then the metric on $V_{2}$ is (the Beltrami) metric

$$
\mathrm{d} s^{2}=\frac{\mathrm{d} \xi^{2}+\mathrm{d} \eta^{2}}{\lambda^{2} \eta^{2}}, \quad k=-\lambda^{2}, \quad \eta>0
$$

see also (A.12). $V_{2}$ is of the type of a Siegel disk $V_{2}=\mathcal{D}_{1} \equiv \mathrm{SU}(1,1) / \mathrm{U}(1)$ or, equivalently, Siegel upper half-plane $\mathcal{H}_{1}$.

For (A.17), see [97, equation (2)] or [64, Theorem 3, p. 644].

The formulation of the following proposition is extracted from [65]: 
Proposition A.14. If $\left(V_{3}, g\right)$ is a homogenous space of dimension 3 , then $\operatorname{dim}\left(I\left(V_{3}\right)\right)=6,4$ or 3 .

1. If $\operatorname{dim}\left(I\left(V_{3}\right)\right)=6$, then $V_{3}$ is of the type of the real space forms, i.e., the real Euclidean space $E^{3}$, the sphere $S^{3}(\kappa)$, or the hyperbolic space $\mathcal{H}^{3}(\kappa)$.

2. If $\operatorname{dim}\left(I\left(V_{3}\right)\right)=4$, then $V_{3}$ is either a Riemannian product $\mathcal{H}^{2}(\kappa) \times \mathbb{R}$ or $S^{2}(\kappa) \times \mathbb{R}$, or one of the following Lie groups with left invariant metric: $\mathrm{SU}(2), \widetilde{\mathrm{SL}}(2, \mathbb{R})$ or $H_{1}$, see [83].

3. If $\operatorname{dim}\left(I\left(V_{3}\right)\right)=3$, then $V_{3}$ is a general 3-dimensional Lie group with left-invariant metric, e.g., the Lie group $\mathrm{Sol}_{3}$, i.e., the group with the composition law:

$$
\left(x_{1}, y_{1}, t_{1}\right)\left(x_{2}, y_{2}, t_{2}\right)=\left(x_{1}+\mathrm{e}^{t} x_{2}, y_{1}+\mathrm{e}^{-t} y_{2}, t_{1}+t_{2}\right)
$$

and the left-invariant metric

$$
\mathrm{d} s^{2}=\mathrm{e}^{-2 t} \mathrm{~d} x^{2}+\mathrm{e}^{2 t} \mathrm{~d} y^{2}+\mathrm{d} t^{2} .
$$

The above classification contains the eight model geometries of Thurston [99]: $E^{3}, \mathcal{H}^{3}, S^{3}$, $\mathcal{H}^{2} \times \mathbb{R}, S^{2} \times \mathbb{R}, \tilde{\mathrm{SL}}(2, \mathbb{R}), H_{1}$ and $\mathrm{Sol}_{3}$.

Cartan classified all 3-dimensional spaces $V_{3}$ with a 4-dimensional isometry group $G_{4}$ in [48], see also [41] and [104]. See also [91] for a modern presentation of Cartan approach.

The Bianchi-Cartan-Vranceanu (BCV) spaces are $V_{3}$ spaces with $\operatorname{dim}\left(I\left(V_{3}\right)\right)=4$ together with $E^{3}$ and $S^{3}(\kappa)$, while the hyperbolic space $\mathcal{H}^{3}(\kappa)$ appearing in Theorem A.11 - a symmetric naturally reductive - is missing in the list of $\mathrm{BCV}$-spaces.

For $\kappa, \tau \in \mathbb{R}$, it is defined the open subset of $\mathbb{R}^{3}$

$$
\operatorname{BCV}(\kappa, \tau):=\left\{(x, y, z) \in \mathbb{R}^{3} \mid D=D(x, y, z ; \kappa)>0, \text { where } D:=1+\frac{\kappa}{4}\left(x^{2}+y^{2}\right)\right\},
$$

equipped with the metric

$$
\mathrm{d} s_{\mathrm{BCV}}^{2}(x, y, z ; \kappa, \tau)=\frac{\mathrm{d} x^{2}+\mathrm{d} y^{2}}{D^{2}}+\left(\mathrm{d} z+\tau \frac{y \mathrm{~d} x-x \mathrm{~d} y}{D}\right)^{2} .
$$

Following [101, Section 2.5] and [47, Example 2.1.10, p. 59], the BCV spaces are described as in

Theorem A.15. All 3-dimensional homogenous spaces $V_{3}$ with isometry group $G_{4}$ are locally isomorphic with the BCV-spaces. The BCV family also includes two real space forms, with isometry group $G_{6}$, see Proposition A.14. The full classification of these spaces is as follows:

1) if $\kappa=\tau=0$, then $\operatorname{BCV}(\kappa, \tau) \cong E^{3}$;

2) if $\kappa=4 \tau \neq 0$, then $\operatorname{BCV}(\kappa, \tau) \cong S^{3}\left(\frac{\kappa}{4}\right) \backslash\{\infty\}$;

3) if $\kappa>0$ and $\tau=0$, then $\left.\operatorname{BCV}(\kappa, \tau) \cong S^{2}(\kappa) \backslash\{\infty\}\right) \times \mathbb{R}$;

4) if $\kappa<0$ and $\tau=0$, then $\operatorname{BCV}(\kappa, \tau) \cong \mathcal{H}^{2}(\kappa) \times \mathbb{R}$;

5) if $\kappa>0$ and $\tau \neq 0$, then $\mathrm{BCV}(\kappa, \tau) \cong \mathrm{SU}(2) \backslash\{\infty\}$;

6) if $\kappa<0$ and $\tau \neq 0$, then $\operatorname{BCV}(\kappa, \tau) \cong \widetilde{\mathrm{SL}}(2, \mathbb{R})$;

7) if $\kappa=0$ and $\tau \neq 0$, then $\mathrm{BCV}(\kappa, \tau) \cong \mathrm{Nil}_{3}$.

Here the Poincaré (Siegel) disc is

$$
\mathcal{H}^{2}(\kappa) \cong\left\{(x, y) \in \mathbb{R}^{2} \mid D<0, \mathrm{~d} s^{2}=\frac{\mathrm{d} x^{2}+\mathrm{d} y^{2}}{D^{2}}\right\} .
$$


An orthonormal frame of vectors on $\mathrm{BCV}(\kappa, \tau)$ is given by

$$
e_{1}=D \frac{\partial}{\partial x}-\tau y \frac{\partial}{\partial z}, \quad e_{2}=D \frac{\partial}{\partial y}+\tau x \frac{\partial}{\partial z}, \quad e_{3}=\frac{\partial}{\partial z},
$$

verifying the commutation relations

$$
\left[e_{1}, e_{2}\right]=\frac{\kappa}{2}\left(-y e_{1}+x e_{2}\right)+2 \tau e_{3}, \quad\left[e_{2}, e_{3}\right]=\left[e_{3}, e_{1}\right]=0 .
$$

The dual 1-forms $\omega_{i},\left\langle\omega^{i} \mid e_{j}\right\rangle=\delta_{i j}, i, j=1,2,3$, to the orthonormal vector fields (A.20) are

$$
\omega^{1}=\frac{\mathrm{d} x}{D}, \quad \omega^{2}=\frac{\mathrm{d} y}{D}, \quad \omega^{3}=\mathrm{d} z+\tau \frac{y \mathrm{~d} x-x \mathrm{~d} y}{D},
$$

and we write down (A.19) as

$$
\mathrm{d} s_{\mathrm{BCV}}^{2}=\sum_{i=1}^{3} \omega^{i} \otimes \omega^{i} .
$$

Let $\mathcal{D}$ be a distribution generated by $e_{1}, e_{2}$. The intrinsic (extrinsic) ideal is given by $\mathcal{J}=\left\langle\omega_{3}\right\rangle$ (respectively, $\mathcal{I}=\left\langle\omega_{1}, \omega_{2}\right\rangle$ ).

If $\tau \neq 0$, the distribution is step 2 everywhere and $\omega^{3}$ is a contact form. If we consider the sub-Riemannian metric

$$
\mathrm{d} s_{\mathcal{D}}^{2}=\sum_{i=1}^{2} \omega^{i} \otimes \omega^{i},
$$

then the $B C V$-space is a sub-Riemannian manifold $\left(\mathrm{BCV}, \mathcal{D}, \mathrm{d} s_{\mathcal{D}}^{2}\right)$.

Remark A.16. Note that the BCV metrics appearing in Cases 1, 2, 5, 6, 7 are metrics on the corresponding naturally reductive spaces of Theorem A.11. Note that naturally reductive space $\mathcal{H}^{3}$ in Theorem A.11, corresponding to the isometry group of dimension 6 , is not a BCV space.

See [58] for a generalization of BCV spaces to 7 dimensions.

Applying the Cayley transform, we can formulate Theorem A.15 on the Siegel upper halfplane, instead on the Siegel disk $D(x, y, z ; \kappa)$ defined by (A.18). We get

Remark A.17. With the Cayley transform

$$
\sqrt{-\frac{\kappa}{4}} \zeta=\frac{v-\mathrm{i}}{v+\mathrm{i}}
$$

we get for (A.18)

$$
D(x, y, z ; \kappa)=1+\frac{4}{\kappa}\left(x^{2}+y^{2}\right)=4 \frac{\operatorname{Im} v}{|v+\mathrm{i}|^{2}}>0,
$$

where $\zeta:=x+\mathrm{i} y$. If $v:=\alpha+\mathrm{i} \beta, E:=\alpha^{2}+(\beta+1)^{2}$, then the left invariant one-forms (A.21) in the new variables are

$$
\begin{aligned}
& \omega^{1}=\frac{\sqrt{-\kappa}}{4} \frac{\left(-\alpha^{2}+\beta^{2}+2 \beta+1\right) \mathrm{d} \alpha-2 \alpha(\beta+1) \mathrm{d} \beta}{\beta E}, \\
& \omega^{2}=\frac{\sqrt{-\kappa}}{4} \frac{\left(\alpha^{2}-\beta^{2}-2 \beta-1\right) \mathrm{d} \alpha+2 \alpha \mathrm{d} \beta}{\beta E}, \\
& \omega^{3}=\mathrm{d} z+\frac{2 \tau}{\kappa} \frac{\left(\alpha^{2}-\beta^{2}+1\right) \mathrm{d} \alpha+2 \alpha \beta \mathrm{d} \beta}{\beta E} .
\end{aligned}
$$

Instead of the family of metrics (A.19), we get in Theorem A.15

$$
\mathrm{d} s_{\mathrm{BCV}}^{2}(\alpha, \beta, z ; \kappa, \tau)=-\frac{1}{\kappa} \frac{\mathrm{d} \alpha^{2}+\mathrm{d} \beta^{2}}{\beta^{2}}+\left(\omega^{3}\right)^{2} .
$$




\section{A.7 G.o. spaces}

The natural reductivity is a special case of spaces with a more general property than $(*)$, see [77]: (**) Each geodesic of $(M, g)=G / H$ is an orbit of a one parameter group of isometries $\{\exp t Z\}$, $Z \in \mathfrak{g}$.

Definition A.18. A vector $X \in \mathfrak{g} \backslash\{0\}$ is called a geodesic vector if the curve $\gamma(t)=(\exp t X)(p)$ is a geodesic.

Riemannian homogeneous spaces with property $(* *)$ are called g.o. spaces (g.o. $=$ geodesics are orbits). All naturally reductive spaces are g.o. manifolds.

Kowalski and Vanhacke [77] have proved that

Proposition A.19 (geodesic lemma). On homogeneous Riemannian manifolds $M=G / H$ a vector $X \in \mathfrak{g} \backslash\{0\}$ is geodesic if and only if

$$
B\left([X, Y]_{\mathfrak{m}}, X_{\mathfrak{m}}\right)=0, \quad \forall Y \in \mathfrak{m} .
$$

It is known, cf. [77]:

Theorem A.20. Every simply connected Riemannian g.o. space $(G / H, g)$ of dimension $n \leq 5$ is a naturally reductive Riemannian manifold.

Kowalski and Szenteke [75] proved that

Theorem A.21. Any homogeneous Riemannian manifold admits at least one homogeneous geodesic through every point $o \in M$.

More details on g.o. spaces and examples are given in [54].

\section{B Balanced metrics and Berezin quantization}

In our approach to Berezin quantization on Kähler manifold $M$ of complex dimension $n$, see, e.g., [21], we considered the Kähler two-form

$$
\omega_{M}(z)=\mathrm{i} \sum_{\alpha, \beta=1}^{n} h_{\alpha \bar{\beta}}(z) \mathrm{d} z_{\alpha} \wedge \mathrm{d} \bar{z}_{\beta}, \quad h_{\alpha \bar{\beta}}=\bar{h}_{\beta \bar{\alpha}}=h_{\bar{\beta} \alpha} .
$$

We have considered homogenous Kähler manifolds $M=G / H$, where the $G$-invariant Kähler two-form is deduced from a Kähler potential $f$

$$
h_{\alpha \bar{\beta}}=\frac{\partial^{2} f}{\partial z_{\alpha} \partial \bar{z}_{\beta}} .
$$

We have applied Berezin recipe to quantization [31, 32, 33, 34], where the Kähler potential is obtained from the scalar product of two Perelomov CS-vectors $e_{z}, z \in M$ [92]

$$
f(z, \bar{z})=\ln K_{M}(z, \bar{z}), \quad K_{M}(z, \bar{z})=\left(e_{\bar{z}}, e_{\bar{z}}\right),
$$

i.e., (1.3).

This choice of $f$ corresponds to the situation where the so called $\epsilon$-function, see [46, 93, 94],

$$
\epsilon(z):=\mathrm{e}^{-f(z)} K_{M}(z, \bar{z}),
$$

is constant. The corresponding $G$-invariant metric is called balanced metric. This denomination was firstly used in [52] for compact manifolds, then it was used in [5] for noncompact manifolds 
and also in [81] in the context of Berezin quantization on homogeneous bounded domain, and we have used it in the case of the partially bounded domain $\mathcal{D}_{n}^{J}$ - the Siegel-Jacobi ball [21].

We recall that in [46, 93, 94] Berezin's quantization on homogenous Kähler manifolds via CS was globalized and extended to non-homogeneous manifolds in the context of geometric (pre-)quantization [73, 107]. To the Kähler manifold $(M, \omega)$, it is also attached the triple $\sigma=(\mathcal{L}, h, \nabla)$, where $\mathcal{L}$ is a holomorphic (prequantum) line bundle on $M, h$ is the Hermitian metric on $\mathcal{L}$ and $\nabla$ is a connection compatible with metric and the Kähler structure [30]. The connection $\nabla$ has the expression $\nabla=\partial+\partial \ln \hat{h}+\bar{\partial}$. The manifold is called quantizable if the curvature of the connection $F(X, Y)=\nabla_{X} \nabla_{Y}-\nabla_{Y} \nabla_{X}-\nabla_{[X, Y]}$ has the property that $F=-\mathrm{i} \omega_{M}$, or $\partial \bar{\partial} \log \hat{h}=\mathrm{i} \omega_{M}$, where $\hat{h}$ is a local representative of $h$, taken $\hat{h}(z)=K_{M}^{-1}(z, \bar{z})$. Then $\omega_{M}$ is integral, i.e., the first Chern class is given by

$$
c_{1}[\mathcal{L}]=\frac{\mathrm{i}}{2 \pi} F=\frac{\omega}{\pi}
$$

and we have (1.3).

\section{Killing vectors on $S^{2}, \mathcal{D}_{1}$ and $\mathbb{R}^{2}$}

\section{C.1 Killing vectors on $S^{2}$}

We consider on the sphere $S^{2}$

$$
\left\{x \in \mathbb{R}^{3} \mid x_{1}^{2}+x_{2}^{2}+x_{3}^{2}=R^{2}\right\}, \quad R>0,
$$

the spherical coordinates, as in Fig. 1 , where $R=1,0 \leq \theta \leq \pi, 0 \leq \varphi \leq 2 \pi$ and

$$
x_{1}=R \sin \theta \cos \varphi, \quad x_{2}=R \sin \theta \sin \varphi, \quad x_{3}=R \cos \theta .
$$

The metric on $S^{2}$ is

$$
\mathrm{d} s_{S^{2}}^{2}(\theta, \varphi)=\mathfrak{g}_{\theta \theta} \mathrm{d} \theta^{2}+g_{\theta \varphi} \mathrm{d} \theta \mathrm{d} \varphi+g_{\varphi \varphi} \mathrm{d} \varphi^{2},
$$

where

$$
g_{\theta \theta}=1, \quad g_{\theta \varphi}=0, \quad g_{\varphi \varphi}=\sin ^{2} \theta, \quad g^{\theta \theta}=1, \quad g^{\theta \varphi}=0, \quad g^{\varphi \varphi}=\frac{1}{\sin ^{2} \theta},
$$

i.e.,

$$
\mathrm{d} s_{S^{2}}^{2}(\theta, \varphi)=\mathrm{d} \theta^{2}+\sin ^{2} \theta \mathrm{d} \varphi^{2} .
$$

We consider a unitary sphere with spherical coordinates (C.1) measured from the origin $O(0,0,0)$. The north (south) pole has coordinates $N(0,0,1)$ (respectively, $S(0,0,-1))$. We take a point $P\left(x_{1}, x_{2}, x_{3}\right)$ on the sphere $S^{2}$ and let $P^{\prime}(\xi, \eta)\left(P^{\prime \prime}\left(\xi^{\prime}, \eta^{\prime}\right)\right)$ be the intersection of the line $N P$ (respectively $S P$ ) with the plane $x_{3}=0$, see Fig. 2 . The triangles $\Delta Q N P$ and $\Delta O N P^{\prime}$ (respectively $\triangle Q P S$ and $\triangle O P^{\prime \prime} S$ ) are similar, and we have

$$
\frac{1-x_{3}}{1}=\frac{x_{1}}{\xi}=\frac{x_{2}}{\eta}, \quad \frac{1+x_{3}}{1}=\frac{x_{1}}{\xi^{\prime}}=\frac{x_{2}}{\eta^{\prime}} .
$$

The change of coordinates $\left(x_{1}, x_{2}, x_{3}\right) \rightarrow(\xi, \eta)$ (respectively, $\left.\left(x_{1}, x_{2}, x_{3}\right) \rightarrow\left(\xi^{\prime} \eta^{\prime}\right)\right)$ is given by the formulas

$$
(\xi, \eta)=\left(\frac{x_{1}}{1-x_{3}}, \frac{x_{2}}{1-x_{3}}\right), \quad\left(\xi^{\prime}, \eta^{\prime}\right)=\left(\frac{x_{1}}{1+x_{3}}, \frac{x_{2}}{1+x_{3}}\right)
$$




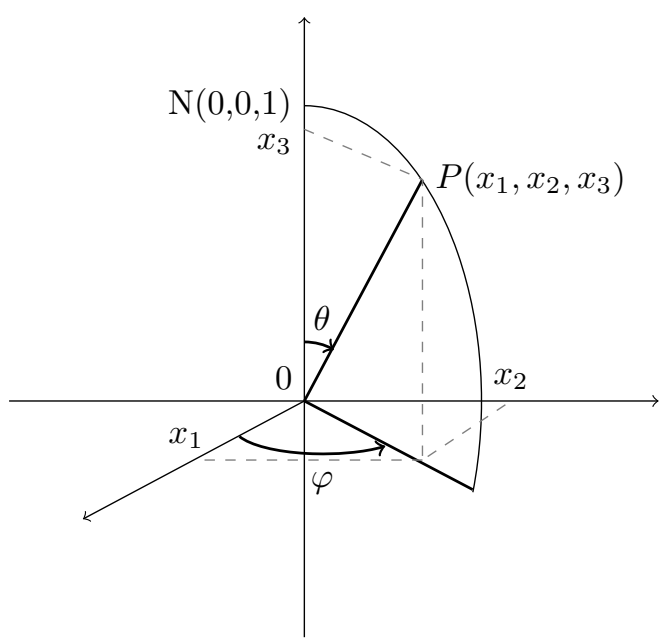

Figure 1. Spherical coordinates.

The change of coordinates $(\xi, \eta) \rightarrow\left(x_{1}, x_{2}, x_{3}\right)$ (respectively, $\left.\left(\xi^{\prime}, \eta^{\prime}\right) \rightarrow\left(x_{1}, x_{2}, x_{3}\right)\right)$ is

$$
\begin{aligned}
& x_{1}=\frac{2 \xi}{1+\xi^{2}+\eta^{2}}, \quad x_{2}=\frac{2 \eta}{1+\xi^{2}+\eta^{2}}, \quad x_{3}=\frac{-1+\xi^{2}+\eta^{2}}{1+\xi^{2}+\eta^{2}}, \\
& x_{1}=\frac{2 \xi^{\prime}}{1+\left(\xi^{\prime}\right)^{2}+\left(\eta^{\prime}\right)^{2}}, \quad x_{2}=\frac{2 \eta^{\prime}}{1+\left(\xi^{\prime}\right)^{2}+\left(\eta^{\prime}\right)^{2}}, \quad x_{3}=\frac{1-\left(\xi^{\prime}\right)^{2}-\left(\eta^{\prime}\right)^{2}}{1+\left(\xi^{\prime}\right)^{2}+\left(\eta^{\prime}\right)^{2}} .
\end{aligned}
$$

Let $\mathbb{C} \ni z:=\xi-\mathrm{i} \eta, z^{\prime}:=\xi^{\prime}+\mathrm{i} \eta^{\prime}$. Then $z z^{\prime}=1$. With (C.3), (C.1), we find

$$
z=\cot \frac{\theta}{2} \mathrm{e}^{-\mathrm{i} \varphi} \text {. }
$$

Introducing (C.4) into the metric on the Riemann sphere

$$
\mathrm{d} s^{2}(z)=4 \frac{\mathrm{d} z \mathrm{~d} \bar{z}}{\left(1+|z|^{2}\right)^{2}}
$$

corresponding to the Kähler two-form (C.5)

$$
\omega=2 j \frac{\operatorname{id} z \wedge \mathrm{d} \bar{z}}{\left(1+|z|^{2}\right)^{2}}
$$

where, if we take $2 j=4$, we get again (C.2).

The equations (A.5) of the covariant components $\left(X_{\theta}, X_{\varphi}\right)$ of the Killing vectors on the sphere $S^{2}$ read

$$
X_{\theta, \theta}=0, \quad X_{\varphi, \varphi}+2 \sin \theta \cos \theta X_{\theta}=0, \quad X_{\theta, \varphi}+X_{\varphi, \theta}-2 \cot \theta X_{\vartheta}=0 .
$$

But

$$
X_{\theta}=g_{\theta \nu} X^{\nu}=X^{\theta}=u, \quad X_{\varphi}=g_{\nu \varphi} X^{\nu}=g_{\varphi \varphi} X^{\varphi}=\sin ^{2} \theta v,
$$

where $\left(X^{\theta}, X^{\varphi}\right)$ are the contravariant components of the Killing vector fields on the sphere $S^{2}$. The equations of the contravariant components of the Killing vector $(u, v):=\left(X^{\theta}, X^{\varphi}\right)$ become

$$
\frac{\partial u}{\partial \theta}=0, \quad \sin ^{2} \theta \frac{\partial v}{\partial \varphi}+\sin \theta \cos \theta u=0, \quad \frac{\partial u}{\partial \varphi}+\frac{\partial v}{\partial \theta}-2 \cot \theta \sin ^{2} \theta v=0 .
$$

We find 


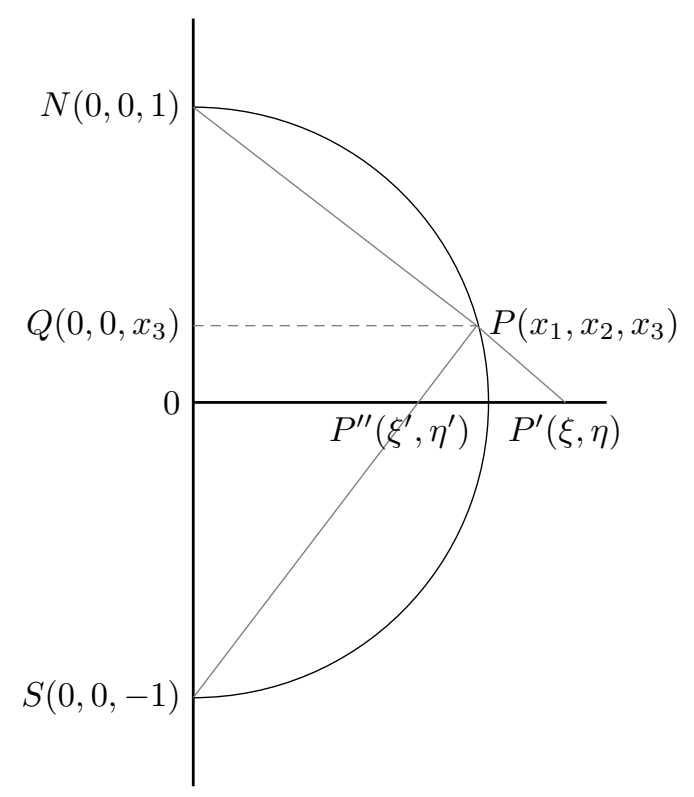

Figure 2. Stereographic projection.

Remark C.1. There are three linearly independent Killing vectors on the sphere $S^{2}$

$$
X=\frac{\partial}{\partial \varphi}, \quad Y=\sin \varphi \frac{\partial}{\partial \theta}+\cos \varphi \cot \theta \frac{\partial}{\partial \varphi}, \quad Z=\cos \varphi \frac{\partial}{\partial \theta}-\sin \varphi \cot \theta \frac{\partial}{\partial \varphi},
$$

which verify the commutation relations

$$
[X, Y]=Z, \quad[Z, X]=Y, \quad[Y, Z]=X .
$$

The Killing vector fields (C.6) in spherical coordinates $(\theta, \varphi)$ on the sphere $S^{2}$ in the stereographic coordinates $(\xi, \eta)$ are

$$
\begin{aligned}
& X=-\eta \frac{\partial}{\partial \xi}+\xi \frac{\partial}{\partial \eta}, \quad Y=\frac{1}{2}\left[2 \xi \eta \frac{\partial}{\partial \xi}+\left(1-\xi^{2}+\eta^{2}\right) \frac{\partial}{\partial \eta}\right] \\
& Z=-\frac{1}{2}\left[\left(1+\xi^{2}-\eta^{2}\right) \frac{\partial}{\partial \xi}+2 \xi \eta \frac{\partial}{\partial \eta}\right] .
\end{aligned}
$$

(C.7) are equations in [104, p. 292]: our $(X, Y, Z)$ in (C.7) correspond to $\left(-Z, \frac{1}{2} Y,-\frac{1}{2} X\right)$, with $m=1$ to formulas of Vranceanu, where the Riemann metric on the Riemann sphere $S^{2}$ is

$$
\mathrm{d} s^{2}=\frac{\mathrm{d} \xi^{2}+\mathrm{d} \eta^{2}}{\left[1+m\left(\xi^{2}+\eta^{2}\right)\right]^{2}} .
$$

\section{C.2 Killing vectors on the Siegel disk $\mathcal{D}_{1}$}

The metric on the Siegel disk $|z|<1$ is

$$
d s^{2}=4 \frac{\mathrm{d} z \mathrm{~d} \bar{z}}{B^{2}}, \quad B=1-|z|^{2}, \quad z=\xi-\mathrm{i} \eta .
$$

The equations (A.4e) of the Killing vectors $u \frac{\partial}{\partial \xi}+v \frac{\partial}{\partial \eta}$ corresponding to the metric (C.9), which are obtained as solution of the equation $L_{X} g=0$, are

$$
\frac{2}{B}(\xi u+\eta v)+\frac{\partial u}{\partial \xi}=0, \quad \frac{2}{B}(\xi u+\eta v)+\frac{\partial v}{\partial \eta}=0, \quad \frac{\partial v}{\partial \xi}+\frac{\partial u}{\partial \eta}=0 .
$$

We find for $\mathcal{D}_{1}$ 
Remark C.2. The Killing vectors on the Siegel disk $\mathcal{D}_{1}$ corresponding to the metric (C.9) are

$$
\begin{aligned}
& X_{1}=\frac{1}{2}\left(\xi^{2}-\eta^{2}-1\right) \frac{\partial}{\partial \xi}+\xi \eta \frac{\partial}{\partial \eta}, \quad Y_{1}=\xi \eta \frac{\partial}{\partial \xi}+\frac{1}{2}\left(\eta^{2}-\xi^{2}-1\right) \frac{\partial}{\partial \eta} \\
& Z_{1}=\eta \frac{\partial}{\partial \xi}-\xi \frac{\partial}{\partial \eta} .
\end{aligned}
$$

The Killing vectors (C.10) on the Siegel disk $\mathcal{D}_{1}$ verify the commutation relations

$$
\left[X_{1}, Y_{1}\right]=-Z_{1}, \quad\left[Y_{1}, Z_{1}\right]=X_{1}, \quad\left[Z_{1}, X_{1}\right]=Y_{1}
$$

\section{C.3 Fundamental vector fields as Killing vector fields on $\mathcal{D}_{1}$ and $\mathcal{X}_{1}$}

We recall some general facts about Hermitian symmetric spaces, see, e.g., [23, 105, 106].

Let

- $X_{n}=G_{n} / K$ : Hermitian symmetric space of noncompact type.

- $X_{c}$ : compact dual form of $X_{n}, X_{c}=G_{c} / K$.

- $G_{n}$ : largest connected group of isometries of $X_{n}$, a centerless semisimple Lie group.

- $G_{c}$ : compact real form of $G_{n}$.

- $G^{c}=G_{n}^{c}=G_{c}^{c}=G$ : complexification of $G_{c}$ and $G_{n}$.

- $K$ : maximal compact subgroup of $G_{n}$.

- $\mathfrak{g}_{n}, \mathfrak{g}, \mathfrak{g}_{c}, \mathfrak{k}$ : Lie algebras of $G_{n}, G, G_{c}, K$ respectively.

- $\mathfrak{g}_{n}=\mathfrak{k}+\mathfrak{m}_{n}$, sum of +1 and -1 eigenspaces of the Cartan involution $\sigma$.

- $\mathfrak{g}=\mathfrak{g}_{n}^{c}=\mathfrak{k}^{c}+\mathfrak{m}$ : complexification, where $\mathfrak{m}=\mathfrak{m}_{n}^{c}$.

- $\mathfrak{g}_{c}=\mathfrak{k}+\mathfrak{m}_{c}$ : compact real form of $\mathfrak{g}_{n}$, where $\mathfrak{m}_{c}=\mathfrak{i m}_{n}$.

We consider the simple Lie algebra $\mathfrak{s l}(2, \mathbb{C})=\langle F, G, H\rangle_{\mathbb{C}}$, whose generators verify the commutation relations

$$
[F, G]=H, \quad[G, H]=2 G, \quad[H, F]=2 F .
$$

We consider the following matrix realization of the $\mathfrak{s l}(2, \mathbb{C})$ algebra

$$
F=e_{12}=\left(\begin{array}{ll}
0 & 1 \\
0 & 0
\end{array}\right), \quad G=e_{21}=\left(\begin{array}{ll}
0 & 0 \\
1 & 0
\end{array}\right), \quad H=e_{11}-e_{22}=\left(\begin{array}{cc}
1 & 0 \\
0 & -1
\end{array}\right) .
$$

To the complex Lie algebra $A_{1}=\mathfrak{s l}(2, \mathbb{C})$ are associated the compact real form $\mathfrak{s l}(2, \mathbb{C})_{c}=\mathfrak{s u}(2)$ and the non-compact real forms $\mathfrak{s u}(1,1)$ and $\mathfrak{s l}(2, \mathbb{R})$, see [63, pp. 186, 446], [23, 105, 106], and we have

$$
\begin{aligned}
& \mathfrak{s u}(2)=\langle\mathrm{i} H,-F+G, \mathrm{i}(F+G)\rangle_{\mathbb{R}}, \\
& \mathfrak{s u}(1,1)=\langle\mathrm{i} H, \mathrm{i}(F-G), F+G\rangle_{\mathbb{R}}, \\
& \mathfrak{s l}(2, \mathbb{R})=\langle H,-F+G, F+G\rangle_{\mathbb{R}} .
\end{aligned}
$$

We have also the isomorphisms between the compact real forms

$$
\mathfrak{s u}(2) \sim \mathfrak{s o}(3) \sim \mathfrak{s p}(1),
$$

and the non-compact real forms

$$
\mathfrak{s l}(2, \mathbb{R}) \sim \mathfrak{s u}(1,1) \sim \mathfrak{s o}(2,1) \sim \mathfrak{s p}(1, \mathbb{R}) .
$$


We have also the relations

$$
\begin{aligned}
& \mathfrak{s u}(1,1)=\mathfrak{g}_{n}=\mathfrak{k}+\mathfrak{m}_{n}, \quad \mathfrak{k}=\mathrm{i} H, \quad \mathfrak{m}_{n}=\langle\mathrm{i}(F-G), F+G\rangle_{\mathbb{R}}, \\
& \mathfrak{s u}(2)=\mathfrak{g}_{c}=\mathfrak{k}+\mathfrak{m}_{\mathfrak{c}}, \quad \mathfrak{m}_{c}=\langle F-G, \mathrm{i}(F+G)\rangle_{\mathbb{R}}, \\
& \mathfrak{s l}(2, \mathbb{C})=\mathfrak{g}=\mathfrak{s u}(1,1)^{c}=\mathfrak{s u}(2)^{c}=\mathfrak{k}^{c}+\mathfrak{m}_{n}^{c}, \quad \mathfrak{k}^{c}=\langle H\rangle_{\mathbb{C}}, \quad \mathfrak{m}_{n}=\langle F, G\rangle_{\mathbb{C}}
\end{aligned}
$$

We calculate the fundamental vector fields for the real noncompact group $\mathrm{SU}(1,1)$. Let us denote the elements of the Lie algebra $\mathfrak{s u}(1,1)$ as

$$
\begin{aligned}
& G_{1}:=\mathrm{i} H=\mathrm{i}\left(\begin{array}{cc}
1 & 0 \\
0 & -1
\end{array}\right), \quad G_{2}:=\mathrm{i}(F-G)=\mathrm{i}\left(\begin{array}{cc}
0 & 1 \\
-1 & 0
\end{array}\right), \\
& G_{3}:=F+G=\left(\begin{array}{ll}
0 & 1 \\
1 & 0
\end{array}\right) .
\end{aligned}
$$

Note the commutation relations

$$
\left[G_{1}, G_{2}\right]=-2 G_{3}, \quad\left[G_{2}, G_{3}\right]=2 G_{1}, \quad\left[G_{3}, G_{1}\right]=-2 G_{2} .
$$

If we make the notation $G_{i}=2 G_{i}^{\prime}, i=1,2,3$, then the commutation relations (C.17) became

$$
\left[G_{1}^{\prime}, G_{2}^{\prime}\right]=-G_{3}^{\prime}, \quad\left[G_{2}^{\prime}, G_{3}^{\prime}\right]=G_{1}^{\prime}, \quad\left[G_{3}^{\prime}, G_{1}^{\prime}\right]=-G_{2}^{\prime}
$$

We obtain, see also [102, p. 294],

$$
\mathrm{e}^{t G_{1}}=\left(\begin{array}{cc}
\mathrm{e}^{\mathrm{i} t} & 0 \\
0 & \mathrm{e}^{-\mathrm{i} t}
\end{array}\right), \quad \mathrm{e}^{t G_{2}}=\left(\begin{array}{cc}
\cosh t & \mathrm{i} \sinh t \\
-\mathrm{i} \sinh t & \cosh t
\end{array}\right), \quad \mathrm{e}^{t G_{3}}=\left(\begin{array}{cc}
\cosh t & \sinh t \\
\sinh t & \cosh t
\end{array}\right) .
$$

We get

$$
\left.\frac{\partial\left(\mathrm{e}^{t G_{1}} \cdot w\right)}{\partial t}\right|_{t=0}=2 \mathrm{i} w,\left.\quad \frac{\partial\left(\mathrm{e}^{t G_{2}} \cdot w\right)}{\partial t}\right|_{t=0}=\mathrm{i}\left(1+w^{2}\right),\left.\quad \frac{\partial\left(\mathrm{e}^{t G_{3}} \cdot w\right)}{\partial t}\right|_{t=0}=1-w^{2} .
$$

With (C.19), we get the corresponding holomorphic fundamental vector fields on the Siegel disk $\mathcal{D}_{1}=\frac{\mathrm{SU}(1,1)}{\mathrm{U}(1)}$ :

$$
G_{1}^{*}=2 \mathrm{i} w \frac{\partial}{\partial w}, \quad G_{2}^{*}=\mathrm{i}\left(1+w^{2}\right) \frac{\partial}{\partial w}, \quad G_{3}^{*}=\left(1-w^{2}\right) \frac{\partial}{\partial w} .
$$

If we introduce $w=\xi-\mathrm{i} \eta$, we write (C.20) as

$$
\begin{aligned}
& G_{1}^{*}=Z_{1}+\mathrm{i}\left(\xi \frac{\partial}{\partial \xi}+\eta \frac{\partial}{\partial \eta}\right), \quad G_{2}^{*}=Y_{1}+\frac{\mathrm{i}}{2}\left[\left(1+\xi^{2}-\eta^{2}\right) \frac{\partial}{\partial \xi}+2 \xi \eta \frac{\partial}{\partial \eta}\right], \\
& G_{3}^{*}=-X_{1}+\frac{\mathrm{i}}{2}\left[2 \xi \eta \frac{\partial}{\partial \xi}+\left(1-\xi^{2}+\eta^{2}\right) \frac{\partial}{\partial \eta}\right]
\end{aligned}
$$

where $X_{1}, Y_{1}, Z_{1}$ are the Killing vector fields of the Siegel disk $\mathcal{D}_{1}$ calculated in (C.10).

We also have the relations, see also [102, p. 353]

$$
\begin{aligned}
& \mathrm{e}^{t F}=\left(\begin{array}{ll}
1 & t \\
0 & 1
\end{array}\right), \quad \mathrm{e}^{t G}=\left(\begin{array}{cc}
1 & 0 \\
t & 1
\end{array}\right), \quad \mathrm{e}^{t H}=\left(\begin{array}{cc}
\mathrm{e}^{t} & 0 \\
0 & \mathrm{e}^{-t}
\end{array}\right), \\
& \mathrm{e}^{t(F+G)}=\left(\begin{array}{cc}
\cosh t & \sinh t \\
\sinh t & \cosh t
\end{array}\right), \quad \mathrm{e}^{t(F-G)}=\left(\begin{array}{cc}
\cos t & \sin t \\
-\sin t & \cos t
\end{array}\right), \\
& \mathrm{e}^{t F} \cdot \tau=\tau+t, \quad \mathrm{e}^{t G} \cdot \tau=\frac{\tau}{1+t \tau}, \quad \mathrm{e}^{t H} \cdot \tau=\mathrm{e}^{2 t} \tau,
\end{aligned}
$$




$$
\begin{aligned}
& \left.\frac{\mathrm{d}}{\mathrm{d} t} \mathrm{e}^{t F} \cdot \tau\right|_{t=0}=1,\left.\quad \frac{\mathrm{d}}{\mathrm{d} t} \mathrm{e}^{t G} \cdot \tau\right|_{t=0}=-\tau^{2},\left.\quad \frac{\mathrm{d}}{\mathrm{d} t} \mathrm{e}^{t H} \cdot \tau\right|_{t=0}=2 \tau, \\
& F^{*}=\partial_{\tau}, \quad G^{*}=-\tau^{2} \partial_{\tau}, \quad H^{*}=2 \tau \partial_{\tau} .
\end{aligned}
$$

If we put $\tau=x+\mathrm{i} y$, we find the fundamental vector fields on the homogenous manifold $\mathcal{X}_{1}$, see Theorem A.10(1) and (4.23)

$$
F_{1}^{*}=\frac{\partial}{\partial x}, \quad G_{1}^{*}=\left(y^{2}-x^{2}\right) \frac{\partial}{\partial x}-2 x y \frac{\partial}{\partial y}, \quad H_{1}^{*}=2\left(x \frac{\partial}{\partial x}+y \frac{\partial}{\partial y}\right) .
$$

In the convention of Section 1 , the vector fields $F_{1}^{*}, G_{1}^{*}, H_{1}^{*}$ are $\mathbb{F}, \mathbb{G}, \mathbb{H}$.

If

$$
A=\left(\begin{array}{ll}
a & b \\
c & d
\end{array}\right) \in \mathrm{SL}(2, \mathbb{R})
$$

then, with formula (C.25),

$$
\operatorname{Ad}(g) X=g X g^{-1}, \quad g \in G, \quad X \in \mathfrak{g},
$$

we find easily

$$
\begin{aligned}
& \operatorname{Ad}(A) F=a^{2} F-c^{2} G-a c H, \\
& \operatorname{Ad}(A) G=-b^{2} F+d^{2} G+b d H, \\
& \operatorname{Ad}(A) H=-2 a b F+2 c d G+(a d+b c) H .
\end{aligned}
$$

We find out that in the base (C.12)

$$
\operatorname{Ad}(A)=\left(\begin{array}{ccc}
a^{2} & -c^{2} & -a c \\
-b^{2} & d^{2} & b d \\
-2 a b & 2 c d & a d+b c
\end{array}\right)
$$

and $\operatorname{det}(\mathrm{Ad})=1$,

Now let us consider an element $X \in \mathfrak{s l}(2, \mathbb{R})$

$$
X=a H+b F+c G=\left(\begin{array}{cc}
a & b \\
c & -a
\end{array}\right) .
$$

Then we find

$$
[X, H]=-2 b F+2 c G, \quad[X, F]=2 a F-c H, \quad[X, G]=-2 a G+b H .
$$

With (C.28) we find in the base $H, F, G$ the expression of $\operatorname{ad}(X)$ for $X$ given by (C.27)

$$
\operatorname{ad}(X)=\left(\begin{array}{ccc}
0 & -2 b & 2 c \\
-2 c & 2 a & 0 \\
b & 0 & -2 a
\end{array}\right),
$$

and

$\operatorname{Tr} \operatorname{ad}=0$.

(C.29) implies, see also [63, p. 551]:

$$
K(X, X)=\operatorname{Tr}(\operatorname{ad} X \circ \operatorname{ad} X)=8\left(a^{2}+b c\right)=4 \operatorname{Tr}(X X) .
$$


As in Remark C.3, we consider

$$
X=a X_{1}+b X_{2}+c X_{3} \in \mathfrak{s u}(2),
$$

where, as in (C.13a),

$$
X_{1}=\mathrm{i} H, \quad X_{2}=-F+G, \quad X_{3}=\mathrm{i}(F+G) .
$$

Then

$$
\operatorname{ad} X=\left(\begin{array}{ccc}
0 & -2 c & 2 b \\
2 c & 0 & -2 a \\
-2 b & 2 a & 0
\end{array}\right)
$$

and

$$
K(X, Y)=-4\left(a a^{\prime}+b b^{\prime}+c c^{\prime}\right),
$$

i.e., the Killing form for $\mathrm{SU}(2)$ is $K(X, Y)=4 \operatorname{Tr}(X Y)$.

Note that for $\mathfrak{s u}(2)$, we have $\mathfrak{m}=\left\langle X_{2}, X_{3}\right\rangle$.

Putting together (C.16)-(C.30) and (C.31), we have proved

Remark C.3. With (C.16), (C.12), (2.2), we get

$$
K_{0}=-\frac{\mathrm{i}}{2} G_{1}, \quad K_{+}=\frac{1}{2}\left(G_{2}+\mathrm{i} G_{3}\right), \quad K_{-}=-\frac{1}{2}\left(G_{2}-\mathrm{i} G_{3}\right) .
$$

Introducing in (C.20) and (C.32), we get the holomorphic fundamental vector fields

$$
K_{0}^{*}=w \frac{\partial}{\partial w}, \quad K_{+}^{*}=\mathrm{i} \frac{\partial}{\partial w}, \quad K_{-}^{*}=-\mathrm{i} w^{2} \frac{\partial}{\partial w}, \quad w \in \mathbb{C}, \quad|w|<1 .
$$

Note that the vector fields $\operatorname{Re} G *_{i}, i=1,2,3$ verify the commutation relations (C.18) with the sign -, i.e., the (real) Killing vector fields $Z_{1}, Y_{1},-X_{1}$ on $\mathcal{D}_{1}$ are the real part of the fundamental vector fields $G_{1}^{\prime *}, G_{2}^{\prime *}, G_{3}^{\prime *}$, corresponding to the metric (C.9).

The fundamental vector fields $F_{1}^{*}, G_{1}^{*}, H_{1}^{*}$ associated to the generators $F, G, H$ (C.12) of $\mathfrak{s l}(2, \mathbb{C})$, given by (C.23), verify the commutation relations (C.11) with a minus sign. They are Killing vector fields corresponding to the Killing equation

$$
-X^{2}+y \partial_{x} X^{1}=0, \quad \partial_{x} X^{2}+\partial_{y} X^{1}=0, \quad-X^{2}+y \partial_{y} X^{2}=0
$$

associated to the metric

$$
c_{1} \frac{\mathrm{d} x^{2}+\mathrm{d} y^{2}}{4 y^{2}}, \quad c_{1}>0
$$

on the Siegel upper half-plane $\mathcal{X}_{1}, x, y \in \mathbb{R}, y>0$.

If $A \in \mathrm{SL}(2, \mathbb{R})$ has the expression (C.24), then the expression of $\operatorname{Ad}(A)$ with respect to the base $F, G, H$ (C.12) is (C.26), and the group $\mathrm{SL}(2, \mathbb{R})$ is unimodular.

The ad matrix in the base $H, F, G$ of $\mathfrak{s l}(2, \mathbb{R})$ is given by $(\mathrm{C} .29)$. The Killing form for $\mathfrak{s l}(2, \mathbb{R})$ is

$$
K(X, Y)=4 \operatorname{Tr}(X Y) .
$$

The Killing form (C.33) is $\mathrm{SL}(2, \mathbb{R})$-invariant and verifies (A.9). Note that

$$
K(H, H)=K(F, G)=4,
$$

and $K(X, Y)=0$ for all $X, Y \in \mathfrak{s l}(2, \mathbb{R})$ different of the choice in (C.34).

The Killing form for the compact group $\mathrm{SU}(2)$ is $K(X, Y)=4 \operatorname{Tr}(X Y)$, and $\mathfrak{m}=\left\langle X_{2}, X_{3}\right\rangle$. 


\section{C.4 Killing vectors on $\mathbb{R}^{2}$}

The Perelomov's coherent state vectors (Glauber's coherent states) for the oscillator group are, see, e.g., [12],

$$
e_{z}:=\mathrm{e}^{z a^{\dagger}} e_{0}
$$

and the scalar product is

$$
\left(e_{\bar{z}}, e_{\bar{z}^{\prime}}\right)=\mathrm{e}^{z \bar{z}^{\prime}}
$$

The scalar product (C.35) of Glauber coherent states on $\mathbb{C}$ implies the metric on $\mathbb{R}^{2}$ (A.14) $\mathrm{d} s_{\mathbb{R}^{2}}^{2}=\mathrm{d} x_{1}^{2}+\mathrm{d} x_{2}^{2}$, where we have considered $z=x_{1}+\mathrm{i} x_{2}$.

Let as consider a vector field on $\mathbb{R}^{2}$

$$
X=X^{1} \frac{\partial}{\partial x_{1}}+X^{2} \frac{\partial}{\partial x_{2}}
$$

We formulate a remark, see also in [57, Section 4.6.7, p. 83]:

Remark C.4. The Killing vectors on $\mathbb{R}^{2}$ associated with the metric (A.14) are

$$
A X+B Y+C Z
$$

where

$$
X=-x_{2} \frac{\partial}{\partial x_{1}}+x_{1} \frac{\partial}{\partial x_{2}}, \quad Y=\frac{\partial}{\partial x_{1}}, \quad Z=\frac{\partial}{\partial x_{2}},
$$

verifying the commutation relations

$$
[X, Y]=-Z, \quad[Y, Z]=0, \quad[Z, X]=-Y
$$

$-X$ is a rotation around $(0,0) \in \mathbb{R}^{2} . Y(Z)$ represents a translation around the $x_{1}$ (respectively $x_{2}$ ) axis. The Killing vectors (C.37) can be put into correspondence with matrix representation (C.38)

$$
a_{1}=\left(\begin{array}{ccc}
0 & 0 & 1 \\
0 & 0 & 0 \\
0 & 0 & 0
\end{array}\right), \quad a_{2}=\left(\begin{array}{ccc}
0 & 0 & 0 \\
0 & 0 & 1 \\
0 & 0 & 0
\end{array}\right), \quad a_{3}=\left(\begin{array}{ccc}
0 & 1 & 0 \\
-1 & 0 & 0 \\
0 & 0 & 0
\end{array}\right)
$$

of the Lie algebra $\mathfrak{e}(2)$ in the representation (C.39)

$$
g=\left(\begin{array}{ccc}
\cos \theta & -\sin \theta & a \\
\sin \theta & \cos \theta & b \\
0 & 0 & 1
\end{array}\right), \quad \theta \in[0,2 \pi), \quad(a, b) \in \mathbb{R}^{2}
$$

of the group $E(2)$.

The Lie algebra of the Killing vectors of $\mathbb{R}^{2}$ with the Euclidean metric (A.14) is $\iota\left(\mathbb{R}^{2}\right)=$ $\mathbb{R}^{2} \rtimes \mathfrak{s o}(2)$, and the Euclidean group $E(2)$ of the plane $\mathbb{R}^{2}$ is $E(2)=\mathbb{R}^{2} \rtimes O(2)$. 


\section{Sasaki manifolds}

\section{D.1 Contact structures}

\section{D.1.1 Maurer-Cartan equations}

Let $G$ be a Lie group with Lie algebra $\mathfrak{g}$, which has the generators $X_{1}, \ldots, X_{n}$ verifying the commutation relations (A.1). To $X \in \mathfrak{g}$ we associate the left-invariant vector $\tilde{X}$ on $G$ such that $\tilde{X}_{e}=X$, see [63, p. 99].

Let $\omega_{1}, \ldots, \omega_{n}$ be the 1 -forms on $G$ determined by the equations $\left\langle\omega_{i} \mid \tilde{X}_{j}\right\rangle=\delta_{i j}, i, j=1, \ldots, n$. Then we have the Maurer-Cartan equations, see, e.g., [63, Proposition 7.2, p. 137]:

$$
\mathrm{d} \omega_{i}=-\frac{1}{2} \sum_{j, k=1}^{n} c_{j k}^{i} \omega_{j} \wedge \omega_{k},
$$

where $c_{j k}^{i}$ are the structure constants (A.1).

If $G$ is embedded in $\mathrm{GL}(n)$ by a matrix valued map $g=(g)_{i j}, i, j=1, \ldots, n$, then let $\lambda$ $(L)$ denote a left-invariant one-form (vector field) on $G$ and $\rho(R)$ a right-invariant one-form (respectively, vector field) on $G$. We have the relations

$$
\begin{aligned}
& g^{-1} \mathrm{~d} g=X_{i} \lambda_{i}, \quad \mathrm{~d} g g^{-1}=X_{i} \rho_{i}, \\
& \left\langle\lambda_{a} \mid L_{b}\right\rangle=\delta_{a b}, \quad\left\langle\rho_{a} \mid R_{b}\right\rangle=\delta_{a b}, \\
& \mathrm{~d} \lambda_{a}=-\frac{1}{2} c_{b c}^{a} \lambda_{b} \wedge \lambda_{c}, \quad \mathrm{~d} \rho_{a}=\frac{1}{2} c_{b c}^{a} \rho_{b} \wedge \rho_{c}, \\
& {\left[L_{a}, L_{b}\right]=c_{a b}^{c} L_{c}, \quad\left[R_{a}, R_{b}\right]=-c_{a b}^{c} R_{c} .}
\end{aligned}
$$

\section{D.1.2 Almost contact manifolds}

Following Sasaki [95] and [45, Definition 6.2.5], we use

Definition D.1. Let $M_{m}$ be a $m=(2 n+1)$-dimensional manifold. $M_{m}$ has a (strict) almost contact structure $(\Phi, \xi, \eta)$ (or $(\xi, \eta, \Phi)$ ) if there exists a $(1,1)$-tensor field $\Phi$, a contravariant vector field (Reeb vector field, or characteristic vector field) $\xi$, and a one-form $\eta$

$$
\Phi=\Phi_{j}^{i} \frac{\partial}{\partial x^{i}} \otimes \mathrm{d} x^{j}, \quad \xi=\xi^{i} \frac{\partial}{\partial x^{i}}, \quad \eta=\eta_{i} \mathrm{~d} x^{i},
$$

verifying the relations

$$
\begin{aligned}
& \langle\eta \mid \xi\rangle=1, \quad \text { or } \quad \eta\lrcorner \xi=1, \quad \text { or } \quad \eta \xi=1, \quad \text { or } \quad \begin{array}{l}
\xi^{i} \eta_{i}=1, \\
\Phi^{2} X=-X+\eta(X) \xi \quad \text { or } \quad \Phi^{2}=-\mathbb{1}_{m}+\xi \otimes \eta,
\end{array} \quad \text { or } \quad \Phi_{j}^{i} \Phi_{k}^{j}=-\delta_{k}^{i}+\xi^{i} \eta_{k},
\end{aligned}
$$

where we have used the convention

$$
\begin{aligned}
& \xi^{t}=\left(\xi^{1}, \ldots, \xi^{m}\right) \in M(1, m, \mathbb{R}), \quad \eta=\left(\eta_{1}, \ldots, \eta_{m}\right) \in M(1, m, \mathbb{R}), \\
& \Phi=\left(\Phi_{j}^{i}\right) \in M(m, \mathbb{R}) .
\end{aligned}
$$

Manifolds $M$ with a structure $(\Phi, \xi, \eta)$ as in Definition (D.3) are called almost contact manifolds.

Sasaki has proved, see [95, Theorem 1.1] and [96, equation (5.16)]: 
Theorem D.2. For an almost contact structure $(\Phi, \xi, \eta)$, the following relations hold

$$
\Phi_{j}^{i} \xi^{j}=0, \quad \text { or } \quad \Phi \xi=0, \quad \Phi_{j}^{i} \eta_{i}=0, \quad \text { or } \quad \eta \Phi=0, \quad \operatorname{Rank}\left(\Phi_{j}^{i}\right)=2 n .
$$

Let $M_{2 n+1}$ be a differentiable manifold with almost contact structure $(\Phi, \xi, \eta)$. Then there exists a positive Riemannian metric $g$ such that

$$
\begin{aligned}
& g(\xi, X)=\eta(X), \quad \text { or } \quad \eta_{i}=g_{i j} \xi^{j}, \quad \text { or } \quad \eta^{t}=g \xi, \\
& g(\Phi X, \Phi Y)=g(X, Y)-\eta(X) \eta(Y), \quad \text { or } \quad \Phi_{h}^{i} g_{i j} \Phi_{k}^{j}=g_{h k}-\eta_{h} \eta_{k}, \quad \text { or } \\
& \Phi^{t} g \Phi=g-\eta^{t} \otimes \eta .
\end{aligned}
$$

If we put

$$
\hat{\Phi}_{i j}:=g_{i h} \Phi_{j}^{h}, \quad \text { or } \quad \hat{\Phi}:=g \Phi,
$$

then $\hat{\Phi}_{i j}=-\hat{\Phi}_{j i}$.

$\hat{\Phi}_{i j}$ is called the associated skew-symmetric tensor of the almost contact metric structure, see also (D.7) below.

\section{D.1.3 Contact structures}

Following [44], we define

Definition D.3. Let $M_{2 n+1}$ be a $C^{\infty}$-manifold of dimension $(2 n+1)$. A contact structure can be given by a codimension one subbundle $\mathcal{D}$ of the tangent bundle $T M$ which is as far from being integrable as possible.

Alternatively, the codimension one subbundle $\mathcal{D}$ of $T M$ can be given as the kernel of a smooth 1-form $\eta$ - the contact form, $\mathcal{D}:=\operatorname{Ker}(\eta)$ - which satisfies the condition

$$
\eta \wedge(\mathrm{d} \eta)^{n} \neq 0
$$

and from (D.6) it follows that the distribution $\mathcal{D}$ is not integrable.

$\mathcal{D}$ is called the contact distribution of the strict contact manifold $(M, \eta)$. A contact structure on $M$ is an equivalence class of such 1-forms, where $\eta^{\prime} \sim \eta$ if there is a nowhere vanishing function on $M$ such that $\eta^{\prime}=f \eta$, cf. [45, Definition 6.1.7].

The tangent space of $M$ has the orthogonal decomposition, see, e.g., [67, p. 9],

$$
T M=\mathcal{D} \oplus\langle\xi\rangle, \quad \text { where } \quad \mathcal{D}=\operatorname{Ann}(\eta)=\{X \in \mathrm{TM} \mid \eta(X)=0\} .
$$

Remark D.4. The codimension one subbundle $\mathcal{D}=\operatorname{Ker}(\eta)$ of $T M$ has an almost complex structure $J=\left.\Phi\right|_{\mathcal{D}}$.

Boothby and Wang [43] have defined

Definition D.5. A contact manifold $M$ is said to be homogeneous if there is a connected Lie group $G$ acting transitively and effectively as a group of differentiable homeomorphisms on $M$ which leave $\eta$ invariant.

If the 1-form $\eta$ has the expression given in (D.2), then

$$
\mathrm{d} \eta=\hat{\Phi}_{i j} \mathrm{~d} x^{i} \wedge \mathrm{d} x^{j}, \quad \text { where } \quad-2 \hat{\Phi}_{i j}=\partial_{i} \eta_{j}-\partial_{j} \eta_{i} .
$$

Note that in [95, equation (3.4)] the minus sign was omitted.

Sasaki has proved, see [95, Theorem 3.1]: 
Theorem D.6. Let $M_{2 n+1}$ be a differentiable manifold with $\eta$ the contact form. Then we can find an almost contact metric structure $(\Phi, \xi, \eta, g)$ such that

$$
\mathrm{d} \eta(X, Y)=g(X, \Phi(Y))
$$

i.e., (D.5) is verified with $\hat{\Phi}$ given by (D.7).

$M_{2 n+1}$ from Theorem D.6 is said to be a contact (Riemannian) manifold associated with $\eta$.

\section{D.2 Structures on cones}

Following [45, p. 201], we define

Definition D.7. Let $(M, g)$ be a smooth Riemannian manifold and let us consider the cone $C(M):=M \times \mathbb{R}^{+}$endowed with the Riemannian metric

$$
\bar{g}=\mathrm{d} r^{2}+r^{2} g, \quad r \in \mathbb{R}^{+} .
$$

$(C(M), \bar{g})$ is called the Riemannian cone (or metric cone) on $M$.

Let $M$ be endowed with the almost contact structure $(\Phi, \xi, \eta)$. Let us define a section $\bar{\Phi}$ of the endomorphism bundle of the $T C(M)=T M \oplus T \mathbb{R}^{+}$as

$$
\bar{\Phi} Y=\Phi Y+\eta(Y) \Psi, \quad \bar{\Phi} \Psi=-\xi, \quad \text { where } \quad Y \in T M, \quad \Psi=r \frac{\partial}{\partial r} \in T \mathbb{R}^{+} .
$$

Then

Remark D.8. In the notation (D.8), $\bar{\Phi}$ defines an almost complex structure on $T C(M)$.

Let

$$
\omega:=\mathrm{d}\left(r^{2} \eta\right)
$$

In accord with [45, Proposition 6.5.5], we have a symplectization (or symplectification) of $M$ :

Proposition D.9. There is one-to-one correspondence between the contact metric structures on $(M, \xi, \eta, g, \Phi)$ and the almost Kähler structures $(C(M), \omega, \bar{g}, \bar{\Phi})$.

According to [45, Definitions 6.4.7, 6.5.7 and 6.5.13]:

Definition D.10. An almost contact structure $(\xi, \eta, \Phi)$ is normal if the corresponding structure $\bar{\Phi}$ on $C(M)$ is integrable. A normal contact metric structure $\mathcal{S}=(M, \xi, \eta, \Phi, g)$ is called a Sasakian structure. $M$ has a $K$-contact structure if $\xi$ is a Killing vector for $g$.

Following [42, p. 47], let us introduce

Definition D.11. Let $h$ be a tensor field of type $(1,1)$. Then the Nijenhuis torsion $[h, h]$ of $h$ is the tensor field of type $(1,2)$ given by

$$
[h, h](X, Y)=h^{2}[X, Y]+[h X, h Y]-h[h X, Y]-h[X, h Y]
$$

Let us define the $(1,2)$-tensor

$$
N^{1}:=[\Phi, \Phi]+2 \mathrm{~d} \eta \otimes \xi .
$$

According with [45, Theorem 6.5.9]: 
Theorem D.12. An almost contact structure $(\xi, \eta, \Phi)$ on $M$ is normal if and only if $N^{1}=0$. Then $(C(M), \bar{g}, \omega, \bar{\Phi})$ is Kähler.

Lemma D.13. The components of the tensor (D.9) are given by

$$
\left(N^{1}\right)_{j k}^{i}=\Phi_{j}^{h} \frac{\partial \Phi_{k}^{i}}{\partial x^{h}}-\Phi_{k}^{h} \frac{\partial \Phi_{j}^{i}}{\partial x^{h}}+\Phi_{h}^{i}\left(\frac{\partial \Phi_{j}^{h}}{\partial x^{k}}-\frac{\partial \Phi_{k}^{h}}{\partial x^{j}}\right)+2 \hat{\Phi}_{j k} \xi^{i} .
$$

Proof. In the calculation below we use the expressions

$$
\begin{aligned}
& \Phi X=(\Phi X)^{i} \frac{\partial}{\partial x^{i}}=\Phi_{j}^{i} X^{j} \frac{\partial}{\partial x^{i}} \\
& {[X, Y]=[X, Y]^{j} \frac{\partial}{\partial x^{j}}=\left(X^{i} \frac{\partial Y^{j}}{\partial x^{i}}-Y^{i} \frac{\partial X^{j}}{\partial x^{i}}\right) \frac{\partial}{\partial x^{j}}} \\
& N^{1}(X, Y)=\left(N^{1}\right)_{j k}^{i} X^{j} Y^{k} \frac{\partial}{\partial x^{i}} .
\end{aligned}
$$

Let us introduce the notation

$$
\begin{aligned}
& A:=\Phi^{2}[X, Y], \quad B:=[\Phi X, \Phi Y], \quad C:=\Phi[\Phi X, Y], \\
& D:=\Phi[X, \Phi Y], \quad E:=2 \mathrm{~d} \eta \otimes \xi(X, Y) .
\end{aligned}
$$

With (D.11), we get for $A, \ldots, E$ the expressions

$$
\begin{aligned}
& A=\Phi(\Phi[X, Y])^{i} \frac{\partial}{\partial x^{i}}=\Phi_{b}^{a}(\Phi[X, Y])^{b} \frac{\partial}{\partial x^{i}}=\Phi_{b}^{a} \Phi_{k}^{b}[X, Y]^{k} \frac{\partial}{\partial x^{a}} \\
&=\left(-\delta_{k}^{a}+\xi^{a} \eta_{k}\right)[X, Y]^{k} \frac{\partial}{\partial x^{a}}, \\
& B= {[\Phi X, \Phi Y]^{a} \frac{\partial}{\partial x^{a}}=\left[(\Phi X)^{c} \frac{\partial(\Phi Y)^{a}}{\partial x^{c}}-(\Phi Y)^{c} \frac{\partial(\Phi X)^{a}}{\partial x^{c}}\right] \frac{\partial}{\partial x^{a}} } \\
&= {\left[X^{d} Y^{b}\left(\Phi_{d}^{c} \frac{\partial \Phi_{b}^{a}}{\partial x^{c}}-\Phi_{b}^{c} \frac{\partial \Phi_{d}^{a}}{\partial x^{c}}\right)+\Phi_{d}^{c} \Phi_{b}^{a}\left(X^{d} \frac{\partial Y^{b}}{\partial x^{c}}-Y^{d} \frac{\partial X^{b}}{\partial x^{c}}\right)\right] \frac{\partial}{\partial x^{a}}, } \\
& C= \Phi_{j}^{a}[\Phi X, Y]^{j} \frac{\partial}{\partial x^{a}}=\Phi_{j}^{a}\left(\Phi_{k}^{i} X^{k} \frac{\partial Y^{j}}{\partial x^{i}}-Y^{i} \frac{\partial\left(\Phi_{h}^{j} X^{h}\right)}{\partial x^{i}}\right) \frac{\partial}{\partial x^{a}}, \\
&-C-D=\left[\Phi_{j}^{a} \Phi_{k}^{i}\left(Y^{k} \frac{\partial X^{j}}{\partial x^{i}}-X^{k} \frac{\partial Y^{j}}{\partial x^{i}}\right)+\left(-\delta_{k}^{a}+\xi^{a} \eta_{k}\right)[Y, X]^{k}\right. \\
&\left.\quad+Y^{i} X^{k} \Phi_{j}^{a}\left(\frac{\partial \Phi_{k}^{j}}{\partial x^{i}}-\frac{\Phi_{i}^{j}}{\partial x^{k}}\right)\right] \frac{\partial}{\partial x^{a}}, \\
& E=2 \hat{\Phi}_{i j} X^{i} Y^{j} \xi^{a} \frac{\partial}{\partial x^{a}} .
\end{aligned}
$$

Introducing the values of $A, B, D+C$ and $E$ obtained in equations (D.12), (D.13), (D.15), respectively (D.16), we get for $A+B-C-D+E$ the values given in (D.10).

Note that formula given in $[95$, pp. $7-10]$

$$
\left(N^{1}\right)_{j k}^{i}=\Phi_{k}^{h}\left(\partial_{h} \Phi_{j}^{i}-\partial_{j} \Phi_{h}^{i}\right)-\Phi_{j}^{h}\left(\partial_{h} \Phi_{k}^{i}-\partial_{k} \Phi_{h}^{i}\right)+\left(\partial_{j} \xi^{i}\right) \eta_{k}-\left(\partial_{k} \xi^{i}\right) \eta_{j}
$$

is wrong. The same wrong formula appears also in [98, equation (3.7)].

The Heisenberg group $H_{1}$ is a Sasaki manifold [44]. 


\section{Acknowledgements}

This research was conducted in the framework of the ANCS project programs PN 164201 01/2016, 180901 01/2018, 190601 01/2019. I had the idea to apply Lemma A.19 after the talk of Professor Zdaněk Dušek at the 1st International Conference on Differential Geometry (April 11-15, 2016, Fez, Morocco). I am grateful to Professor Zdaněk for his correspondence in the first stages of the preparation of this paper. I also would like to thank Professor Mohamed Tahar Kadaoul Abbassi for the hospitality during the Fez conference and the partial financial support. I would like to thank to Professor G.W. Gibbons for answering to an e-mail. I am grateful to Professor M. Visinescu for initiating me in the world of Sasaki manifolds. Thanks are also addressed to Professor R.D. Grigore for suggestions in some calculations. I am grateful to Professors Dmitri Alekseevsky and Vicente Cortés for criticism and suggestions on the first version of this paper. The author thanks the unknown referees' who through their recommendations contributed to the improvement of the text of the paper. The author thanks Drs. I. Berceanu and M. Babalic for help in preparation of the text.

\section{References}

[1] Agricola I., Ferreira A.C., Friedrich T., The classification of naturally reductive homogeneous spaces in dimensions $n \leq 6$, Differential Geom. Appl. 39 (2015), 59-92, arXiv:1407.4936.

[2] Albert C., Le groupe de Heisenberg et les variétés de contact riemanniennes, Bull. Sci. Math. 126 (2002), 97-113.

[3] Alekseevskii D.V., Reductive space, Encyclopedia of Mathematics, https://www. encyclopediaofmath.org/ /index.php?title=Reductive_space\&oldid=33884.

[4] Ambrose W., Singer I.M., On homogeneous Riemannian manifolds, Duke Math. J. 25 (1958), 647-669.

[5] Arezzo C., Loi A., Moment maps, scalar curvature and quantization of Kähler manifolds, Comm. Math. Phys. 246 (2004), 543-559.

[6] Arvanitoyeorgos A., An introduction to Lie groups and the geometry of homogeneous spaces, Student Mathematical Library, Vol. 22, Amer. Math. Soc., Providence, RI, 2003.

[7] Bargmann V., Group representations on Hilbert spaces of analytic functions, in Analytic Methods in Mathematical Physics (Sympos., Indiana Univ., Bloomington, Ind., 1968), 1970, 27-63.

[8] Berceanu S., Coherent states and geodesics: cut locus and conjugate locus, J. Geom. Phys. 21 (1997), 149-168, arXiv:dg-ga/9502007.

[9] Berceanu S., Coherent states, phases and symplectic areas of geodesic triangles, in Coherent States, Quantization and Gravity, Editors M. Schlichenmaier, A. Strasburger, S.T. Ali, A. Odjziewicz, Warsaw University Press, Warsaw, 2001, 129-137, arXiv:math.DG/9903190.

[10] Berceanu S., Geometrical phases on Hermitian symmetric spaces, in Recent Advances in Geometry and Topology, Cluj University Press, Cluj-Napoca, 2004, 83-98, arXiv:math.DG/0408233.

[11] Berceanu S., A holomorphic representation of Lie algebras semidirect sum of semisimple and Heisenberg algebras, Romanian J. Phys. 50 (2005), 81-94.

[12] Berceanu S., Realization of coherent state Lie algebras by differential operators, in Advances in Operator Algebras and Mathematical Physics, Theta Ser. Adv. Math., Vol. 5, Theta, Bucharest, 2005, 1-24, arXiv:math.DG/0504053.

[13] Berceanu S., A holomorphic representation of the Jacobi algebra, Rev. Math. Phys. 18 (2006), 163-199, Errata, Rev. Math. Phys. 24 (2012), 1292001, 2 pages, arXiv:math.DG/0408219.

[14] Berceanu S., Coherent states associated to the Jacobi group, Romanian Rep. Phys. 59 (2007), $1089-1101$.

[15] Berceanu S., Coherent states associated to the Jacobi group - a variation on a theme by Erich Kähler, J. Geom. Symmetry Phys. 9 (2007), 1-8.

[16] Berceanu S., A holomorphic representation of the multidimensional Jacobi algebra, in Perspectives in Operator Algebras and Mathematical Physics, Theta Ser. Adv. Math., Vol. 8, Theta, Bucharest, 2008, 1-25, arXiv:math.DG/0604381. 
[17] Berceanu S., A convenient coordinatization of Siegel-Jacobi domains, Rev. Math. Phys. 24 (2012), 1250024, 38 pages, arXiv:1204.5610.

[18] Berceanu S., Consequences of the fundamental conjecture for the motion on the Siegel-Jacobi disk, Int. J. Geom. Methods Mod. Phys. 10 (2013), 1250076, 18 pages, arXiv:1110.5469.

[19] Berceanu S., Coherent states and geometry on the Siegel-Jacobi disk, Int. J. Geom. Methods Mod. Phys. 11 (2014), 1450035, 25 pages, arXiv:1307.4219.

[20] Berceanu S., Bergman representative coordinates on the Siegel-Jacobi disk, Romanian J. Phys. 60 (2015), 867-896, arXiv:1409.0368.

[21] Berceanu S., Balanced metric and Berezin quantization on the Siegel-Jacobi ball, SIGMA 12 (2016), 064, 28 pages, arXiv:1512.00601.

[22] Berceanu S., Geodesics associated to the balanced metric on the Siegel-Jacobi ball, Romanian J. Phys. 61 (2016), 1137-1160, arXiv:1605.02962.

[23] Berceanu S., Boutet de Monvel L., Linear dynamical systems, coherent state manifolds, flows, and matrix Riccati equation, J. Math. Phys. 34 (1993), 2353-2371.

[24] Berceanu S., Gheorghe A., On the construction of perfect Morse functions on compact manifolds of coherent states, J. Math. Phys. 28 (1987), 2899-2907.

[25] Berceanu S., Gheorghe A., On equations of motion on compact Hermitian symmetric spaces, J. Math. Phys. 33 (1992), 998-1007.

[26] Berceanu S., Gheorghe A., Linear Hamiltonians on homogeneous Kähler manifolds of coherent states, An. Univ. Timişoara Ser. Mat.-Inform. 39 (2001), 31-55, arXiv:math.DG/0408254.

[27] Berceanu S., Gheorghe A., Differential operators on orbits of coherent states, Romanian J. Phys. 48 (2003), 545-556, arXiv:math.DG/0211054.

[28] Berceanu S., Gheorghe A., Applications of the Jacobi group to quantum mechanics, Romanian J. Phys. 53 (2008), 1013-1021, arXiv:0812.0448.

[29] Berceanu S., Gheorghe A., On the geometry of Siegel-Jacobi domains, Int. J. Geom. Methods Mod. Phys. 8 (2011), 1783-1798, arXiv:1011.3317.

[30] Berceanu S., Schlichenmaier M., Coherent state embeddings, polar divisors and Cauchy formulas, J. Geom. Phys. 34 (2000), 336-358, arXiv:math.DG/9903105.

[31] Berezin F.A., Quantization in complex bounded domains, Dokl. Akad. Nauk SSSR 211 (1973), 1263-1266.

[32] Berezin F.A., Quantization, Math. USSR-Izv. 8 (1974), 1109-1165.

[33] Berezin F.A., General concept of quantization, Comm. Math. Phys. 40 (1975), 153-174.

[34] Berezin F.A., Quantization in complex symmetric spaces, Math. USSR-Izv. 9 (1975), 341-379.

[35] Berndt J., Tricerri F., Vanhecke L., Generalized Heisenberg groups and Damek-Ricci harmonic spaces, Lecture Notes in Mathematics, Vol. 1598, Springer-Verlag, Berlin, 1995.

[36] Berndt J., Vanhecke L., Naturally reductive Riemannian homogeneous spaces and real hypersurfaces in complex and quaternionic space forms, in Differential Geometry and its Applications (Opava, 1992), Math. Publ., Vol. 1, Silesian University Opava, Opava, 1993, 353-364.

[37] Berndt R., Some differential operators in the theory of Jacobi forms, preprint IHES/M/84/10, 1984, 31 pages.

[38] Berndt R., Sur l'arithmétique du corps des fonctions elliptiques de niveau $N$, in Seminar on Number Theory, Paris 1982-83 (Paris, 1982/1983), Progr. Math., Vol. 51, Birkhäuser Boston, Boston, MA, 1984, 21-32.

[39] Berndt R., Schmidt R., Elements of the representation theory of the Jacobi group, Progress in Mathematics, Vol. 163, Birkhäuser Verlag, Basel, 1998.

[40] Besse A.L., Einstein manifolds, Ergebnisse der Mathematik und ihrer Grenzgebiete (3), Vol. 10, SpringerVerlag, Berlin, 1987.

[41] Bianchi L., Sugli spazi a tre dimensioni che ammettono un gruppo continuo di movimenti, Mem. Mat. Fis. Soc. Ital. Sci. 11 (1898), 267-352, On the three-dimensional spaces which admit a continuous group of motions, Gen. Relativity Gravitation 33 (2001), 2171-2253.

[42] Blair D.E., Contact manifolds in Riemannian geometry, Lecture Notes in Math., Vol. 509, Springer-Verlag, Berlin - New York, 1976.

[43] Boothby W.M., Wang H.C., On contact manifolds, Ann. of Math. 68 (1958), 721-734. 
[44] Boyer C.P., The Sasakian geometry of the Heisenberg group, Bull. Math. Soc. Sci. Math. Roumanie (N.S.) 52 (2009), 251-262.

[45] Boyer C.P., Galicki K., Sasakian geometry, Oxford Mathematical Monographs, Oxford University Press, Oxford, 2008.

[46] Cahen M., Gutt S., Rawnsley J., Quantization of Kähler manifolds. II, Trans. Amer. Math. Soc. 337 (1993), $73-98$.

[47] Calin O., Chang D.-C., Sub-Riemannian geometry. General theory and examples, Encyclopedia of Mathematics and its Applications, Vol. 126, Cambridge University Press, Cambridge, 2009.

[48] Cartan E., Leçons sur la Géométrie des Espaces de Riemann, Gauthier-Villars, Paris, 1928.

[49] Cartan E., La méthode du repére mobile, la théorie des groupes continus et les espaces généralisés, Actualités scientifiques et industrielles, Vol. 194, Hermann \& Cie., Paris, 1935.

[50] Cartan E., Les espaces à connexion projective, Abh. Sem. Vektor-Tensor analysis, Moskau 4 (1937), 147173.

[51] D'Atri J.E., Ziller W., Naturally reductive metrics and Einstein metrics on compact Lie groups, Mem. Amer. Math. Soc. 18 (1979), iii+72 pages.

[52] Donaldson S.K., Scalar curvature and projective embeddings. I, J. Differential Geom. 59 (2001), 479-522.

[53] Dorfmeister J., Nakajima K., The fundamental conjecture for homogeneous Kähler manifolds, Acta Math. 161 (1988), 23-70.

[54] Dušek Z., Survey on homogeneous geodesics, Note Mat. 28 (2009), suppl. 1, 147-168.

[55] Eichler M., Zagier D., The theory of Jacobi forms, Progress in Mathematics, Vol. 55, Birkhäuser Boston, Inc., Boston, MA, 1985.

[56] Evtushik E.L., Moving-frame method, Encyclopedia of Mathematics, http://www.encyclopediaofmath. org/index.php?title=Moving-frame-method\&oldid=17828.

[57] Fecko M., Differential geometry and Lie groups for physicists, Cambridge University Press, Cambridge, 2006.

[58] Ferrández A., Naveira A.M., Tarrío A.D., Geometry of extended Bianchi-Cartan-Vranceanu spaces, Rev. R. Acad. Cienc. Exactas Fís. Nat. Ser. A Mat. RACSAM 113 (2019), 1515-1532, arXiv:1802.00106.

[59] Folland G.B., Harmonic analysis in phase space, Annals of Mathematics Studies, Vol. 122, Princeton University Press, Princeton, NJ, 1989.

[60] Freitas P.J., On the action of the symplectic group on the Siegel upper half plane, Ph.D. Thesis, University of Illinois at Chicago, 1999.

[61] Gindikin S.G., Pjateckiı̌-Šapiro I.I., Vinberg E.B., Homogeneous Kähler manifolds, in Geometry of Homogeneous Bounded Domains, Springer, Berlin, Heidelberg, 2011, 1-87.

[62] Halverscheid S., Iannuzzi A., On naturally reductive left-invariant metrics of SL(2, R), Ann. Sc. Norm. Super. Pisa Cl. Sci. (5) 5 (2006), 171-187.

[63] Helgason S., Differential geometry, Lie groups, and symmetric spaces, Pure and Applied Mathematics, Vol. 80, Academic Press, Inc., New York - London, 1978.

[64] Hua L.-K., On the theory of automorphic functions of a matrix level. I. Geometrical basis, Amer. J. Math. 66 (1944), 470-488.

[65] Inoguchi J.-I., Van der Veken J., Parallel surfaces in the motion groups E(1,1) and E(2), Bull. Belg. Math. Soc. Simon Stevin 14 (2007), 321-332.

[66] Itzykson C., Remarks on boson commutation rules, Comm. Math. Phys. 4 (1967), 92-122.

[67] Jayne N., Legendre foliations on contact metric manifolds, Ph.D. Thesis, Massey University, 1992.

[68] Kähler E., Raum-Zeit-Individuum, Rend. Accad. Naz. Sci. XL Mem. Mat. 16 (1992), 115-177.

[69] Kähler E., Mathematische Werke/Mathematical works, Walter de Gruyter \& Co., Berlin, 2003.

[70] Kobayashi S., Nomizu K., Foundations of differential geometry, Vol. I, Interscience Publishers, New York London, 1963.

[71] Kobayashi S., Nomizu K., Foundations of differential geometry, Vol. II, Interscience Publishers, New York London - Sydney, 1969.

[72] Koda T., An introduction to the geometry of homogeneous spaces, in Proceedings of the 13th International Workshop on Differential Geometry and Related Fields, Natl. Inst. Math. Sci. (NIMS), Taejŏn, 2009, 121144 . 
[73] Kostant B., Quantization and unitary representations. I. Prequantization, in Lectures in Modern Analysis and Applications III, Lecture Notes in Math., Vol. 170, Springer, Berlin - Heidelberg, 1970, 87-208.

[74] Kowalski O., Spaces with volume-preserving symmetries and related classes of Riemannian manifolds, Rend. Sem. Mat. Univ. Politec. Torino (1983), 131-158.

[75] Kowalski O., Szenthe J., On the existence of homogeneous geodesics in homogeneous Riemannian manifolds, Geom. Dedicata 81 (2000), 209-214.

[76] Kowalski O., Vanhecke L., Four-dimensional naturally reductive homogeneous spaces, Rend. Sem. Mat. Univ. Politec. Torino (1983), 223-232.

[77] Kowalski O., Vanhecke L., Riemannian manifolds with homogeneous geodesics, Boll. Un. Mat. Ital. B 5 (1991), 189-246.

[78] Lang S., SL $2(\mathbb{R})$, Graduate Texts in Mathematics, Vol. 105, Springer-Verlag, New York, 1985.

[79] Lisiecki W., A classification of coherent state representations of unimodular Lie groups, Bull. Amer. Math. Soc. (N.S.) 25 (1991), 37-43.

[80] Lisiecki W., Coherent state representations. A survey, Rep. Math. Phys. 35 (1995), 327-358.

[81] Loi A., Mossa R., Berezin quantization of homogeneous bounded domains, Geom. Dedicata 161 (2012), 119-128, arXiv:1106.2510.

[82] Mikeš J., Vanžurová A., Hinterleitner I., Geodesic mappings and some generalizations, Palacký University Olomouc, Olomouc, 2009.

[83] Milnor J., Curvatures of left invariant metrics on Lie groups, Adv. Math. 21 (1976), 293-329.

[84] Moscovici H., Coherent state representations of nilpotent Lie groups, Comm. Math. Phys. 54 (1977), 63-68.

[85] Moscovici H., Verona A., Coherent states and square integrable representations, Ann. Inst. H. Poincaré 29 (1978), 139-156.

[86] Neeb K.-H., Coherent states, holomorphic extensions, and highest weight representations, Pacific J. Math. 174 (1996), 497-542.

[87] Neeb K.-H., Holomorphy and convexity in Lie theory, De Gruyter Expositions in Mathematics, Vol. 28, Walter de Gruyter \& Co., Berlin, 2000.

[88] Nomizu K., Invariant affine connections on homogeneous spaces, Amer. J. Math. 76 (1954), 33-65.

[89] Onishchik A.L., Isotropy group, Encyclopedia of Mathematics, https://www.encyclopediaofmath.org/ /index.php?title=Isotropy_group\&_oldid=33104.

[90] Onishchik A.L., Isotropy representation, Encyclopedia of Mathematics, https://www.encyclopediaofmath . org//index . php?title=Isotropy_representation\&oldid=15929.

[91] Patrangenaru V., Classifying 3- and 4-dimensional homogeneous Riemannian manifolds by Cartan triples, Pacific J. Math. 173 (1996), 511-532.

[92] Perelomov A., Generalized coherent states and their applications, Texts and Monographs in Physics, Springer-Verlag, Berlin, 1986.

[93] Rawnsley J., Coherent states and Kähler manifolds, Quart. J. Math. Oxford Ser. (2) 28 (1977), $403-415$.

[94] Rawnsley J., Cahen M., Gutt S., Quantization of Kähler manifolds. I. Geometric interpretation of Berezin's quantization, J. Geom. Phys. 7 (1990), 45-62.

[95] Sasaki S., On almost contact manifolds, Lecture notes, Part 1, Tôhoku University, 1965.

[96] Sasaki S., Hatakeyama Y., On differentiable manifolds with contact metric structures, J. Math. Soc. Japan 14 (1962), 249-271.

[97] Siegel C.L., Symplectic geometry, Amer. J. Math. 65 (1943), 1-86.

[98] Tanno S., The automorphism groups of almost Hermitian manifolds, Trans. Amer. Math. Soc. 137 (1969), 269-275.

[99] Thurston W.P., Three-dimensional geometry and topology, Vol. 1, Princeton Mathematical Series, Vol. 35, Princeton University Press, Princeton, NJ, 1997.

[100] Tricerri F., Vanhecke L., Homogeneous structures on Riemannian manifolds, London Mathematical Society Lecture Note Series, Vol. 83, Cambridge University Press, Cambridge, 1983.

[101] Van der Veken J., Higher order parallel surfaces in Bianchi-Cartan-Vranceanu spaces, Results Math. 51 (2008), 339-359, arXiv:math.DG/0604541. 
[102] Vilenkin N.Ja., Special functions and the theory of group representations, Translations of Mathematical Monographs, Vol. 22, Amer. Math. Soc., Providence, R.I., 1968.

[103] Vinberg E.B., Gindikin S.G., Kähler manifolds admitting a transitive solvable group of automorphisms, Math. USSR Sb. 3 (1967), 333-351.

[104] Vranceanu G., Lecţii de Geometrie diferenţială, Vol. I, Editura Didactică şi Pedagogică, Bucureşti, 1962.

[105] Wolf J.A., The action of a real semisimple group on a complex flag manifold. I. Orbit structure and holomorphic arc components, Bull. Amer. Math. Soc. 75 (1969), 1121-1237.

[106] Wolf J.A., Fine structure of Hermitian symmetric spaces, in Symmetric Spaces (Short Courses, Washington Univ., St. Louis, Mo., 1969-1970), Pure and App. Math., Vol. 8, Editors W.M. Boothby, G.I. Weiss, Marcel Dekker and G.L. Weiss, New York, 1972, 271-357.

[107] Woodhouse N., Geometric quantization, Oxford Mathematical Monographs, The Clarendon Press, Oxford University Press, New York, 1980.

[108] Yang J.-H., The method of orbits for real Lie groups, Kyungpook Math. J. 42 (2002), 199-272, arXiv:math.RT/0602056.

[109] Yang J.-H., Remark on harmonic analysis on Siegel-Jacobi space, arXiv:math.NT/0612230.

[110] Yang J.-H., Invariant metrics and Laplacians on Siegel-Jacobi space, J. Number Theory 127 (2007), 83-102, arXiv:math.NT/0507215.

[111] Yang J.-H., A partial Cayley transform of Siegel-Jacobi disk, J. Korean Math. Soc. 45 (2008), 781-794, arXiv:math.NT/0507216.

[112] Yang J.-H., Invariant metrics and Laplacians on Siegel-Jacobi disk, Chin. Ann. Math. Ser. B 31 (2010), 85-100, arXiv:math.NT/0507217.

[113] Yano K., The theory of Lie derivatives and its applications, Bibliotheca Mathematica, Vol. 3, North-Holland Publishing Company, Amsterdam, 1957. 